NUREG/CR-1298

PNL-3158

\title{
Growth and Histological Effects to Protothaca staminea (Littleneck Clam) of Long-Term Exposure to Chlorinated Sea Water
}

Prepared by C. I. Gibson, R. E. Hillman, P. Wilkinson, D. L. Woodruff

Pacific Northwest Laboratory

Operated by

Battelle Memorial Institute

Prepared for

U.S. Nuclear Regulatory

Commission 


\section{NOTICE}

This report was prepared as an account of work sponsored by an agency of the United States Government. Neither the United States Government nor any agency thereof, or any of their employees, makes any warranty, expressed or implied, or assumes any legal liability or responsibility for any third party's use, or the results of such use, of any information, apparatus product or process disclosed in this report, or represents that its use by such third party would not infringe privately owned rights.

Available from

GPO Sales Program

Division of Technical Information and Document Control

U. S. Nuclear Regulatory Commission

Washington, D. C. 20555

Printed copy price: $\$ 3.75$

and

National Technical Information Service

Springfield, Virginia 22161 
NUREG/CR-1298

PNL-3158

RE

\section{Growth and Histological Effects to Protothaca staminea (Littleneck Clam) of Long-Term Exposure to Chlorinated Sea Water}

Manuscript Completed: June 1980

Date Published: August 1980

Prepared by

C. I. Gibson, R. E. Hiliman, P. Wilkinson, D. L. Woodruff

Pacific Northwest Laboratory

Richland, WA 99352

\section{Prepared for}

Division of Sefeguards, Fuel Cycle and Environmental Research

Office of Nuclear Regulatory Research

U.S. Nuclear Regulatory Commission

Washington, D.C. 20555

NRC FIN No. B2098 



\section{ABSTRACT}

There has been considerable concern about the potential for long-term effects to marine organisms from chlorinated sea water. As part of a larger study to investigate the effects of materials resulting from seawater chlorination on marine organisms, groups of littleneck clams, Protothaca staminea, were exposed to sea water that had been chlorinated. Two experiments were conducted. In one test, groups of littleneck clams were exposed to dilutions of chlorinated sea water that had average chlorine produced oxidant (CPO) concentrations of $16 \mu \mathrm{g} / \boldsymbol{l}$ or less. In the second test, groups of clams were exposed to chlorinated seawaterunchlorinated seawater mixtures that had target CPO concentrations of 0 , $6,12,25,50$ and $100 \mu \mathrm{g} / \ell$. In the first experiment, length measurements were made on all clams at approximately one-month intervals for three months. In the second test, length, weight, depth, width and edge etching were used to measure growth, and subsamples were harvested and measured at one-month intervals. In addition, clams were preserved for histological examination.

The clams in the first experiment all had negative growth. In the second test, growth was inhibited under all conditions through the first four months of exposure. During the last four months, there was positive signs of growth at the 0,6 and $12 \mu \mathrm{g} / \ell$ CPO test conditions.

Histological examination indicates that $P$. staminea does not adapt well to being held in aquaria. Most clams, from ali test and control conditions, showed evidence of necrosis at one month. This condition seemed to improve with longer exposure at lower CPO concentrations but persisted at CPO concentrations of $25 \mu \mathrm{g} / \ell$ and higher. Other histological effects were apparent at the higher exposure concentrations as the length of exposure increased. 



\section{SUMMARY}

Studies of the effects of long-term exposure to chlorinated sea water on the growth of littleneck clams (Protothaca staminea) were initiated in 1977 , as a subtask to the program on the synthesis and effects of halogenated organics created by the chlorination of cooling water at nuclear fueled steam electric stations. The objective of this subtask was to determine the effect on clam growth of exposure to chlorinated sea water. The initial experimental design had five groups of 60 clams being exposed to Sequim Bay sea water that had been chlorinated at a level of approximately $1.5 \mathrm{mgCl} / \ell$ with sodium hypochlorite and then diluted with untreated sea water to obtain $0,2,4,6$ and 12-fold dilutions of the chlorinated sea water. A sixth tank containing $60 \mathrm{clams}$ and recejving untreated sea water was used as a control. Total length measurements were made on the clams at approximately 1-month intervals for a period of three months.

Over the course of the exposure, the total average net growth for all clam groups was negative $(-0.08 \mathrm{~mm}$ to $-0.13 \mathrm{~mm})$. The average $C P 0$ in the tanks during the period ranged from $0.016 \mathrm{mg} / \ell$ to $0.00 \mathrm{mg} / \ell$. The cause of the negative growth in all tanks was not identified, but a number of factors were suggested, e.g., insufficient food supply, routine disturbance, chlorine produced oxidant effects, tank stress and lack of sensitivity of the length measurement over the test period.

A second series of growth experiments were conducted in the winter and spring of 1978, using a new delivery system and protocol. To alleviate what was thought to be factors that may have contributed to the negative growth in the earlier test, feeding with algal culture was planned for this test, and weight, width, thickness, and edge marking were done in addition to the length measurement. In addition, individual clams would be disturbed only at the initiation of the experiment and at harvest time. Finally, clams would be preserved for histological examination for any tissue abnormalities.

The new delivery system delivered a mixture of chiorinated and unchlorinated sea water to the test tanks so that the respective groups of clams had target CPO concentrations of $0,6,12,25,50$ and $100 \mu \mathrm{g} / \ell$. The $0 \mu \mathrm{g} / \mathrm{l}$ test tank received only untreated sea water, and the $100 \mathrm{\mu g} / \ell$ test tank received only chlorinated sea water. The initial chlorination rate was approximately $1.5 \mathrm{mg} / \ell$ chlorine per liter of sea water. Subsamples of clams were harvested from each test tank at approximately 30-day intervals for measurements and preservation for histological examination.

Shell deposition, measured by the etched edge markings, indicated that no growth took place in any of the test tanks until the fifth month at which time there were positive signs of growth in the control and in the 6 and $12 \mu \mathrm{g} / \ell$ test conditions. Positive growth was noted in these tanks after six and eight months also. No signs of positive growth occurred 
at the 25, 50 and $100 \mu \mathrm{CPO} / \mathrm{C}$. The other measurements supported the shell deposition finding but indicated that linear measurement is not a good parameter to use to measure growth in littleneck clams under these conditions.

Histological examination of the clams indicated that the clams were under some stress when collected from the field. However, the clams appeared to recover during the first month of testing, and then the organisms at the higher CPO concentrations (50 and $100 \mu \mathrm{g} / \mathrm{l}$ ) developed tissue abnormalities. 


\section{TABLE OF CONTENTS}

Abstract

Suminary

List of Figures

ix

List of Tables

$x i$

Preface

xvi

Acknowledgments

xix .

Introduction

1

Discussion

4

Conclusions and Recommendations

6

References 


\section{LIST OF FIGURES}

Figure 1. Exposure system used for Protothaca staminea in the first growth experiments

Figure 2. Exposure system used for Protothaca staminea in the second growth experiments

Figure 3. Photomicrographs of sections through the littleneck clam, Protothaca staminea 



\section{LIST OF TABLES}

Table 1. Initial clam length in exposure tank receiving $100 \%$ control sea water. Date: $2 / 1 / 77$

Table 2. Initial clam length in exposure tank receiving 94\% control sea water, $6 \%$ chlorinated sea water.

Date: $2 / 1 / 77$

Table 3. Initial clam length in exposure tank receiving $88 \%$ control sea water, $12 \%$ chlorinated sea water.

Date: $2 / 2 / 77$

Table 4. Initial clam length in exposure tank receiving $75 \%$ control sea water, $25 \%$ chlorinated sea water.

Date: $2 / 2 / 77$

Table 5. Initial clam length in exposure tank receiving 50\% control sea water, $50 \%$ chlorinated sea water.

Date: $2 / 2 / 77$

Table 6. Initial clam length in exposure tank receiving $100 \%$ chlorinated sea water. Date: $2 / 3 / 77$

Table 7. Length of clams in tank receiving $100 \%$ control sea water after 1 month of exposure. Date: $3 / 1 / 77$

Table 8 . Length of clams in tank recejving $94 \%$ control sea water, $6 \%$ chlorinated sea water after 1 month of exposure. Date: $3 / 1 / 77$

Table 9. Length of clams in tank receiving $88 \%$ control sea water, $12 \%$ chlorinated sea water after 1 month of exposure. Date: $3 / 2 / 77$

Table 10 . Length of clams in tank receiving $75 \%$ control sea water, $25 \%$ chlorinated sea water after 1 month of exposure. Date: $3 / 4 / 77$

Table 11. Length of clams in tank receiving $50 \%$ control sea water, $50 \%$ chlorinated sea water after 1 month of exposure. Date: 3/4/77

Table 12. Length of clams in tank receiving $100 \%$ chlorinated sea water after 1 month of expsoure. Date: $3 / 4 / 77$

Table 13. Length of clams added to tanks to replace animals removed for chemical and histological examination. 
Table 14. Length of clams in tank receiving $100 \%$ control sea water after 2 months of exposure. Date: 4/4/77

Table 15. Length of clams in tank receiving $94 \%$ control sea water, $6 \%$ chlorinated sea water after 2 months of exposure. Date: $4 / 5 / 77$

Table 16. Length of clams in tank receiving $88 \%$ control sea water, $12 \%$ chlorinated sea water after 2 months of exposure. Date: $4 / 5 / 77$

Table 17. Length of clams in tank receiving $75 \%$ control sea water, 25\% chiorinated sea water after 2 months of exposure. Date: $4 / 5 / 77$

Table 18. Length of clams in tank receiving $50 \%$ control sea water, $50 \%$ chlorinated sea water after 2 months of exposure. Date: $4 / 6 / 77$

Table 19. Length of clams in tank receiving $100 \%$ chlorinated sea water after 2 months of exposure. Date: 4/6/77

Table 20. Length of clams in tank receiving $100 \%$ control sea water after 3 months of exposure. Date: $5 / 2 / 77$

Table 21 . Length of clams in tank receiving $94 \%$ control sea water, $6 \%$ chlorinated sea water after 3 months of exposure. Date: $5 / 3 / 77$

Table 22. Length of clams in tank receiving $88 \%$ control sea water, $12 \%$ chlorinated sea water after 3 months of exposure. Date: 5/4/77

Table 23. Length of clams in tank receiving $75 \%$ control sea water, 25\% chlorinated sea water after 3 months of exposure. Date $5 / 4 / 77$

Table 24 . Length of clams in tank receiving $50 \%$ control sea water, 50\% chlorinated sea water after 3 months of exposure. Date: 5/5/77

Table 25. Length of clams in tank receiving $100 \%$ chlorinated sea water after 3 months of exposure. Date: 5/5/77

Table 26. Dissolved oxygen, temperature, salinity and $\mathrm{pH}$ of sea water in tank receiving $75 \%$ control sea water and $25 \%$ chlorinated sea water. 
Table 27. Chlorine produced oxidant concentrations in clam exposure tanks ( $\mathrm{mg} / \mathrm{l})$.

Table 28. Length change in clams from tank receiving $100 \%$ control sea water after 3 months of exposure.

Table 29. Length change in clams from tank receiving $94 \%$ control sea water, $6 \%$ chlorinated sea water after 3 months of exposure.

Table 30 . Length change in clams from tank receiving $88 \%$ control sea water, $12 \%$ chlorinated sea water after 3 months of exposure.

Table 31. Length change in clams from tank receiving $75 \%$ control sea water, $25 \%$ chlorinated sea water after 3 months of exposure.

Table 32. Length change in clams from tank receiving $50 \%$ control sea water, $50 \%$ chlorinated sea water after 3 months of exposure.

Table 33. Length change in clams from tank receiving $100 \%$ chlorinated sea water after 3 months of exposure.

Table 34. Summary of length changes at all test conditions after 3 months of exposure.

Table 35. Length, width, thickness, and weight measurements of clams in control exposure tank. Date: $2 / 27 / 78$

Table 36. Length, width, thickness, and weight measurements of clams in $6 \mu \mathrm{g} / \ell$ CPO exposure tank. Date: $2 / 27 / 78$

Table 37. Length, width, thickness, and weight measurements of clams in $12 \mu \mathrm{g} / \ell$ CPO exposure tank. Date: $2 / 27 / 78$

Table 38. Length, width, thickness, and weight measurements of clams in $25 \mu \mathrm{g} / \ell$ CP0 exposure tank. Date: $2 / 27 / 78$

Table 39. Length, width, thickness, and weight measurements of clams in $50 \mu \mathrm{g} / \mathrm{\ell}$. CPO exposure tank. Date: $2 / 27 / 78$

Table 40. Length, width, thickness, and weight measurements of clams in $100 \mu \mathrm{g} / \ell$ CP0 exposure tank. Date: $2 / 27 / 78$

Table 41. Length, width, thickness, and weight measurements of clams after one month of exposure. Date: $4 / 3 / 78$ 
Table 42. Length, width, thickness, and weight measurements of clams after two months of exposure. Date: 5/1/78

Table 43. Length, width, thickness, and weight measurements of clams after three months of exposure. Date: $5 / 30 / 78$

Table 44. Length, width, thickness, and weight measurements of clams after four months of exposure. Date: $6 / 29 / 78$

Table 45. Length, width, thickness, and weight measurements of clams after five months of exposure. Date: $8 / 1 / 78$

Tabje 46. Length, width, thickness, and weight measurements of clams after six months of exposure. Date: $9 / 5 / 78$

Table 47. Length, width, thickness, and weight measurements of clams after eight months of exposure. Date: 11/8/78

Table 48. Measured CPO concentrations $(\mu \mathrm{g} / \ell)$ in exposure tanks.

Table 49. Histopathological descriptions of Protothaca staminea from Sequim Bay, Washington.

Table 50. Histopathological descriptions of Protothaca staminea used as controls in Tank No. I of Chlorine Bioassay Study.

Table 51. Histopathological descriptions of Protothaca staminea exposed to $6 \mathrm{ppb}$ chlorine in Tank No. 2 of ChTorine Bioassay Study.

Table 52. Histopathological description of Protothaca staminea exposed to $12 \mathrm{ppb} c h$ lorine in Tank No. 3 of Chlorine Bioassay Study.

Table 53. Histopathological description of Protothaca staminea exposed to $25 \mathrm{ppb}$ chlorine in Tank No. 4 of Chlorine Bioassay Study.

Table 54. Histopathological descriptions of Protothaca staminea exposed to $50 \mathrm{ppb}$ chlorine in Tank No. 5 of Chlorine Bioassay Study.

Table 55. Histopathological descriptions of Protothaca staminea exposed to $100 \mathrm{ppb}$ chlorine in Tan $\bar{k}$ No. 6 of Chlorine Bioassay Study. 
Table 56. Results of histopathological examination of initial sample of Protothaca staminea collected from Sequim Bay, Washington in March, 1978.

Table 57. Summary of histological descriptions of the 1ittleneck clam, Protothaca staminea, exposed to various amounts of chlorine for one month.

Table 58. Summary of histological descriptions of the littleneck clam, Protothaca staminea, exposed to various amounts of chlorine for two months.

Table 59. Summary of histological descriptions of the littleneck clam, Protothaca staminea, exposed to various amounts of chlorine for three months.

Table 60. Summary of histological descriptions of the littleneck clam, Protothaca staminea, exposed to various amounts of chlorine for four months.

Table 61. Summary of histological descriptions of the littleneck clam, Protothaca staminea, exposed to various amounts of chlorine for five months.

Table 62. Summary of histological descriptions of the littleneck clam, Protothaca staminea, exposed to various amounts of chlorine for six months.

Table 63. Summary of histopathological observations of littleneck clams, Protothaca staminea, exposed to various CPO concentrations for up to $\mathrm{six}$ months.

Table 64. 8romoform concentrations in clams exposed to chlorinated sea water containing sublethal concentrations of chlorine produced oxidants (CPO). 



\section{PREFACE}

This report includes data and analys is for the Marine Biology Task of the program on Biocide By-Products in Aquatic Environments.

Reports prepared for the entire program are:

Title

- Investigation of Halogenated Components Formed from Chlorination of Natural Waters: Preliminary Studies, NUREG/CR-1299

- Acute Toxicity and Bioaccumulation of Chloroform to Four Species of Fresh water Fish Salmo gairdneri, Rainbow Trout Lepomis macrochirus, Bluegill Micropterus saTmoides, Largemouth Bass Ictalurus punctatus, Channel Catfish, NUREG/CR-0893

- Chronic Effects of Chiorination By-Products on Rainbow Trout, Salmo gairdneri, NUREG/CR-0892

- Toxicity, Bioaccumulation and Depuration of Bromoform in Five Marine Species

Protothaca staminea, Littleneck Clam

Mercenaria mercenaria, Eastern Hard Clam, Quahog Crassostrea virginica, Eastern oyster Penaeus aztecus, Brown Shrimp Brevoortia tyrannus, At lantic Menhaden, NUREG/CR-1297

- Growth and Histologica? Effects to Protothaca staminea, (Littleneck Clam) of Long-Term Exposure to Chlorinated Sea Water, NUREG/CR-1298

- Analysis of Organohalogen Products from Chlorination of Natural Waters Under Simulated Biofouling Control Conditions, NUREG/CR-1301

- Biocide By-Products in Aquatic Environments, Final Report Covering Period September 10, 1976 through September 30, 1979, NUREG/CR-1300
Author

Roger M. Bean Robert G. Riley

David R. Anderson E. William Lusty

David R. Anderson Roger M. Bean Roger E. Schirmer

Charles I. Gibson Fredrick C. Tone Peter Wilkinson J. W. Blaylock Roger E. Schirmer

Charles I. Gibson Robert E. Hillman Peter Wilkinson Dana L. Woodruff

Roger M. Bean Dale C. Mann Robert G. Riley

Roger M. Bean Charles I. Gibson David R. Anderson 



\section{ACKNOWLEDGEMENTS}

This work was performed for the U.S. Nuclear Regulatory Commission (NRC) under a Related Services Agreement with the U.S. Department of Energy Contract DE-AC06-76RL0-1830. The author wishes to acknowledge the guidance and assistance provided by $0 r$. Phillip R. Reed of NRC.

Contributors to this report are:

C. I. Gibson

R. E. Hiliman

P. Wilkinson

D. L. Woodruff

T. 0 . Thatcher

R. W. Schirmer
Principal Investigator, Manager, Marine Sciences Section

Scientist

Technical Support Specialist

Scientist

Consultant

Scientist

Trade names and registered trademarks are used to assist the reader in replicating the experiments. Their use does not constitute endorsement by Battelle Memorial Institute or the U.S. NRC. 



\section{INTRODUCTION}

Studies of the effects of long-term exposure to chlorinated sea water on the growth of 1 ittleneck clams (Protothaca staminea) were initiated in 1977 as a subtask to the program on the synthes is and effects of halogenated organics created by the chlorination of cooling water at nuclear fueled steam electric stations. Numerous studies had been conducted on the acute toxicity of chlorine and chlorine produced oxidants ( $\mathrm{CP} 0$ ) to fish and other marine organisms $(1,2,3,4,5,6)$. However, little information was available on the effects of long-term exposure (months) of organisms to CPO. In addition, findings of halogenated organics created by chlorination of fresh and marine waters presented another group of compounds that may cause deleterious effects to exposed organisms $(7,8,9)$.

The objective of the research discussed here was to expose littleneck clams to sea water that had been chlorinated at a rate similar to expected rates at operating steam electric stations. To insure that the clams would not die from the acute toxic effects of CPO, the CPO concentration was reduced by aging (natural demand) and dilution with control sea water. Two tests were conducted.

The first experiment was designed to test the delivery system and look at the response of littleneck clams to long-term holding in laboratory tanks. This experiment was run with set dilutions of the chlorinated sea water delivered to the individual aquaria holding the clams, and only clam length was measured. Based on the results of the first test, a number of modifications were made to the delivery systems and the biological measurements to be collected. Using the modified delivery system, the second test was conducted using target concentrations of CPO of $0,6,12,15,50$, and $100 \mu \mathrm{g} / \ell$.

\section{First Experiment}

Littleneck clams were collected from Kiapot Point, Sequim Bay, Washington on 11/28/76, and held in a large circular tank receiving raw Sequim Bay sea water. Additional food was provided to the clams daily in the form of a slurry of ground Fusia sp., Ulva sp. and alfalfa flour. On 12/15/76, $50 \mathrm{clams}$ were randomly selected and placed in one of the 5 ix $120 \mathrm{\ell}$ glass exposure aquaria (Figure 1). The aquaria contained $12-13 \mathrm{~cm}$ of sand as a substrate for the clams to bury in. The clams were observed for burying activity, and al1 but 2 were buried within 8 hours. On 12/19/76, 50 clams were introduced into each of the remaining five exposure tanks, and chlorination of the sea water was begun. Temperature was maintained at $15^{\circ} \mathrm{C}$. The clams were observed daily and individuals that did not bury or ones that surfaced were replaced. From 12/19/76 through $2 / 1 / 77$, a total of $12 \mathrm{clams}$ were removed (from the 6 tanks) and replaced with new individuals.

on $2 / 1 / 77$, the clams in each tank were removed for measuring and numbering. The procedure was to remove all the clams from a tank, and blow them 
with an air gun until the snells appeared "bone dry." fength measurements were then made and the individuals numbered with Flecto varathane \#101 orange paint. The paint was allowed to dry for six hours before the clams were returned to their respective tanks. The clams were out of the tank for approximately 8 hours.

The tanks were then observed daily for clams that surfaced and died. At monthly intervals the clams were removed and length measurements made (Tables 1 to 25). Also at monthly intervals, 5 clams were removed for future histopathological examination and chemical analysis. To maintain even densities throughout the exposure the removed clans were replaced with new individuals. CPO, temperature, salinity, $\mathrm{pH}$ and dissolved oxygen measurements were made approximately daily (Tables $26 \& 27$ ). CP0 was measured by the potentiometric method (10).

The length measurements indicated that the clams were not growing and appeared to have been experiencing shell erosion or negative growth (Tables 28 to 34 ). Several factors could have contributed to this, including disturbance by the monthly measuring routine, lack of sufficient food, adverse reaction to being confined in the tank, and exposure to chlorinated sea water. Since the control showed the same negative length change, the chlorine exposure could not be singled out as the factor causing growth suppression.

However, the major objective of this experiment was satisfied. That is, it was found that littleneck clams could be held in aquaria for periods of up to 6 months without massive mortalities, and they also could survive in sea water that had been chlorinated at a rate of 1 to $2 \mathrm{mg} / \mathrm{l}$ but had low ( $\leqq 0.02 \mathrm{mg} / \ell$ ) CPO concentrations. The cause of the negative growth could not be related to a single factor, and, because of this, the second experiment included a number of modifications.

\section{Second Experiment}

The next experiment was designed to monitor more parameters that would be indicators of growth and to monitor their health by histological examination. To reduce some of the suspected compounding factors thought to exist in the first experiment, the following changes were made. The clams were not measured every month. Instead, only those individuals that were harvested for other purposes were measured. Additional food, in the form of cultured algae, was provided. Proportional dilutions of

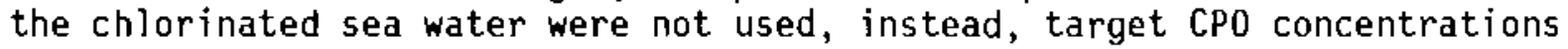
were maintained in each tank. The shell edge was etched to provide a more sensitive indicator of growth. Body measurements, in addition to the length, were taken.

The Mount-Brungs type delivery system used in the first test was replaced by a manifold type system shown in Figure 2. The clam exposure tanks were the same $120 \mathrm{e}$ aquaria used in the initial test and the bottom was covered with $12-13 \mathrm{~cm}$ of sand. The sea water on the chlorinated side 
was chlorinated at a rate of approximately $1.5 \mathrm{mg} / \ell \mathrm{Cl}_{2}$. The exposure tanks received chlorinated and control sea water in proportions that produced the target in tank CPO concentrations. The control tank received $100 \%$ control water, and the $100 \mu \mathrm{g} / \mathrm{l}$ CP0 tank received only chlorinated sea water. The flows to the individual tanks varied in order to maintain the appropriate CPO. The control tank received 450 to $500 \mathrm{ml} / \mathrm{min}$ and the other tanks received at least that amount. In general, the flows remained between 500 and $1000 \mathrm{ml} / \mathrm{min}$.

Clams were collected from Pitship Point, Sequim Bay, Washington and held for marking in running, raw sea water. Clams were randomly selected, marked with a motorized engraver, edge-etched, weighed, measured for length, width and thickness, and placed into the individual exposure tanks. Initial tank loading was $60 \mathrm{clams}$. Ten clams were preserved for histopathological examination, and $10 \mathrm{clams}$ were frozen for chemical analysis.

Feeding was provided by first drawing the water level in the tanks down by removing the stand pipe, then replacing the stand pipe and adding Monochrysis sp. culture to provide a cell density of approximately $200,000 / m \ell$ in the full tank. The exposure tank was then allowed to fill gradually with its normal mixture of control and chlorinated sea water. The control and chlorinated sea water was filtered $(100 \mu \mathrm{m})$ so that the only food received by the clams was through the feeding. After three months, problems developed with the filter apparatus and in the phytoplankton culture, and the exposure system was switched to raw sea water and no feeding.

At approximately one-month intervals, $8 \mathrm{clams}$ were harvested from each tank. The harvesting was done in a manner that caused miminal disturbance to the remaining individuals. The harvested clams were measured and weighed, and checked for positive signs of shell deposition on the etched edge. Four were frozen in glass jars for chemical analys is, and 4 were preserved in Davidson's fixative for histological examination. The measurement and weights are presented in Tables 35 to 47 . The measured CPO concentrations in the tanks are presented in Table 48.

The clams that were fixed in Davidson's fixative were shipped to Battelle's William F. Clapp Laboratories, Duxbury, Massachusetts where they were embedded in paraplast, sectioned at $6 \mu \mathrm{m}$ and stained with hemotoxilin and eosin. The sections were then examined by Dr. Robert E. Hillman. The results of these examinations are presented in Tables 49 to 63 . A summary of these results is provided in Table 63.

The clams that were frozen for chemical examination were shipped frozen to the Battelle Northwest Richland Laboratories for analyses by Dr. Roger Schirmer. As a result of the work done under the analytical portion of this program, these tissues were analyzed for bromoform. other compounds were checked for but only bromoform results are presented in Table 64. 
Analysis of tissues was done by homogenizing the tissue in water at $0^{\circ} \mathrm{C}$ and diluting with enough water to obtain a concentration of approximately one gram of tissue per $10 \mathrm{~m} \ell$ of tissue suspension. Ten to $20 \mathrm{~m} \ell$ aliquots of the aqueous tissue suspension were extracted with two 5 me portions of hexane containing 1-, 3-dibromopropane as an internal standard. The microliter samples of the hexane solution were injected into a gas chromatograph fitted with an $18^{\prime \prime}$ Porapak $Q^{6}$ column and a ${ }^{63} \mathrm{Ni}$ electron capture detector. The column was operated isothermally at $185^{\circ} \mathrm{C}$. The limit of detection of this procedure was $0.0005 \mu \mathrm{g} / \mathrm{g}$, and the coefficient of variation ranged from $1 \%$ at the 1 to $8 \mu \mathrm{g} / \mathrm{g}$ level to $3 \%$ at levels below $0.1 \mu \mathrm{g} / \mathrm{g}$. The coefficient of variation was calculated from 16 replicate analyses of each of 9 tissue samples.

\section{DISCUSSION}

A summary of histological observations is given in Table 63 . Of the 10 clams fixed prior to the start of exposure, 6 had some necrotic tissue, and 2, including one of the necrotic specimens, had metaplastic digestive tubules, with the normally columnar epithelium being reduced to a low cuboidal form (Figure 2). This condition persisted in the 1-month exposure series with most of the clams showing evidence of necrosis; half of them having general necrosis throughout the viscera. Improvement in tissue condition was noted with longer exposure at lower chlorine concentrations, but necrosis persisted at $25 \mu \mathrm{g} / \mathrm{\ell}$ and higher after 2 and 3 months exposure. At 4 months exposure, metaplasia of the digestive tubules increased at 12,25 , and $50 \mu \mathrm{g} / \mathrm{l}$. This condition improved after 5 months but returned after 6 months. Leukocytic infiltration into the tissues increased during the first 2 months and remained at about the same level throughout the 6 months of exposure. There was general necrosis and autolysis of connective tissues after 6 months exposure at 50 and $100 \mu \mathrm{g} / \ell$. Vacuolization of stomach and intestinal epithelium (Figure 3 ) was common in the clams exposed to 50 and $100 \mu \mathrm{g} / \ell$ CP0 after the first 2 months of exposure.

The growth data indicates that under the conditions of the test, clams were not in a very active growth mode. Vanderhorst and Wilkinson (MRL unpublished data) found, in field studies with Protothaca staminea during the active growing season in spring and summer, that the initiation of new shell growth can be very sporadic once the clams are disturbed. It appears that during the study, the clams were slow to initiate new growth and, in fact, by the last harvest date ( 8 months of holding) only $36 \%$ ( 4 of 11 ) in the control had laid down new she 11 . In the two previous harvest dates ( 5 and 6 months), only 1 of 8 (13\%) had she 11 deposition. However, there appears to be a pattern with growth evident at the control and lower two test levels ( $6 \mu \mathrm{g} / \ell$ and $12 \mu \mathrm{g} / \ell \mathrm{CPO}$ ) and no growth at the higher three test concentrations $(25 \mu \mathrm{g} / \ell, 50 \mu \mathrm{g} / \ell$ and $100 \mu \mathrm{g} / \ell$ ). This same pattern is evident from the width and weight data. 
The weight data could be considered the most indicative measure with the fact that shell growth can be very sporadic, particularly after handling. of interest here is the fact that at the control and lower two concentrations there was some positive weight gain in each test and no individuals with weight loss, whereas, at the high concentrations $(25 \mu \mathrm{g} / \ell, 50 \mu \mathrm{g} / \ell$ and $100 \mu \mathrm{g} / \ell$ ) the opposite was true. The histological data indicates that at these higher CPO concentrations the amount of tissue damage, particularly in the stomach, intestine and digestive tubules, is significant and could have reduced the clams' ability to feed and digest food. In addition, the amount of necrosis and autolysis evident at the higher concentrations could lead to premature death.

In the higher CPO concentrations the proportion of food (planktonic organisms) exposed to chlorine was higher than at the lower concentrations and, therefore, could have served as an additional stress on the organisms. If phytoplankton was destroyed by the chlorination process, these clams would have received less food in the last five months which could be a factor in their growth.

Since the clams were collected in March, the initial samples might have been stressed by winter conditions, which could account for the pathological conditions observed in those specimens and the 1-month exposures. Recovery was slower at higher concentrations and considerably inhibited at 50 and $100 \mu \mathrm{g} / \ell$.

The tissue analysis data for bromoform must be viewed with caution because of suspected contamination in the exposure system. During the period of March through June, bromoform was being used in the same room as the long-term chlorinated seawater exposure was being conducted. In another series of tests, we found that it was difficult to obtain a bromoform/seawater solution. Therefore, the possibility of cross contamination by bromoform vapor from the chlorine test system was not considered. The results of the tissue analyses indicate that our assumption was wrong. However, these data do indicate that if bromoform is present, it will be accumulated by the clams.

The results of the second experiment indicate that long-term exposure of littleneck clams to chlorinated sea water with CPO concentrations above $50 \mu \mathrm{g} / \mathrm{\ell}$ has an effect. The effects observed in this test were inhibition of growth, as determined by new shell deposition and weight change, and tissue damage observed by histological examination. From the results it appears that the length measurement used in the first study is the least reliable parameter to use when determining the growth of littleneck clams. The width measurements showed a more consistent trend than the length measurement, for, as the concentration of CPO increased, there was a decrease in the number of clams which increased in width over the 8-month exposure period. The weight measurement provides the most consistent trend, with the number of individuals that were larger at the end of the exposure period being 0 at the three higher CPO concentrations, while the number which decreased in weight was 0 in the control and lowest two concentrations and was 1,6 , and 2 in the three higher exposure 
conditions. It must be noted, however, that the sample size was limited, and further testing is needed to refine the results.

Shell deposition, as indicated by edge etching, appears to be the best sign of positive growth. However, it needs to be coupled with weight gain to provide an indication that the clam was not using stored energy to repair a damaged shell but was healthy and able to add tissue at the same time.

The histological examination of the clams provided the most detailed data for determining their health. However, because of the limited data base on normal clam tissue and the variety of factors that can cause specific histological changes, it is difficult to definitely identify cause and effect at this time. From the results of this study it appears that the higher CPO concentrations had an adverse effect on the clam, and the tissue damage observed could be the reason for no growth at these exposure concentrations. In addition to this apparent effect, the tissue pathology that was present early in the testing in much of the clam population indicates that attention needs to be paid to the initial health of test organisms. Laboratory experiments that are attempting to determine the long-term effect of man-produced stress must be aware of all the compounding factors that can affect the results, and that traditional methods of assessing health may not be enough to te 11 the whole story.

The histological data from these studies provides a good basis for field validation of effects from chlorinated sea water. To determine if a chlorinated discharge is having an effect, the histological condition of organisms being impinged upon by the discharge could be compared to a population outside the discharge's influence but still within the same natural physical, chemical and biological environment.

\section{CONCLUSIONS AND RECOMMENDATIONS}

Under the test conditions used, CPO concentrations of 50 and $100 \mu \mathrm{g} / \ell$ had an adverse effect on the growth of littleneck clams.

Under the test conditions used, the control group and groups exposed to target $C P O$ concentrations of 6,12 , and $25 \mu \mathrm{g} / \ell$ had positive growth.

Histological examination of the clams showed stress conditions at the beginning of the exposure, but the clams in the control and lower CPO concentrations $(6,12$ and $25 \mu \mathrm{g} / 2)$ recovered while those at the higher concentrations (50 and $100 \mu \mathrm{g} / \ell$ ) had significant tissue damage at the end of the test period ( 6 months).

The ultimate consequences of the lack of growth and tissue damage on the ability of the clams to survive and reproduce was not determined.

However, the data indicates that clam populations that are continually exposed to CPO concentrations of $50 \mu \mathrm{g} / \ell$ or higher will be under greater stress than those exposed to concentrations of $25 \mathrm{\mu g} / \ell$ or less.

Field sampling of mollusk populations exposed to CPO should be undertaken to verify the existence of similar tissue damage in the natural environment. 


\section{REFERENCES}

1. Brungs, W. A. "Effects of Residual Chlorine on Aquatic Life," J. Water Pollut. Control Fed. 45(10):2180-2193 (1973).

2. Brungs, W. A. "Effects of Wastewater and Cooling Water Chlorination on Aquatic Life," EPA-600/3-76-098 (Washington, D. C.: U. S. Environmental Protection Agency, 1976).

3. Gibson, C. I., T.0. Thatcher and C. W. Apts. "Some Effects of Tempera" ture, Chlorine and Copper on the Survival and Growth of the Coon Stripe Shrimp," in Thermal Ecology II. Proceedings of 1975 Symposium, Augusta, Georgia, G. W. Esch and R. W. McFarlane, Eds. U. S. ERDA Report No. CONF-750425 (Arlington, Virginia: National Technical Information Service, 1976).

4. Roberts, M. H., Jr., R. Diaz, M. E. Bender and R. Huggett. "Acute Toxicity of Chlorine to Selected Estuarine Species," J. Fish. Res. Board of Can, 32(12): 2525-2528 (1975).

5. Goldman, J. C., J. M. Capuzzo, and G.T.F. Wong. "Biological and Chemical Effects of Chlorination of Coastal Power Plants," in Water Chlorination Environmental Impact and Health Effects, Vol. 2, R. L. Jolley, H. Grochev and D. H. Hamilton, Jr., Eds. (Ann Arbor, Michigan: Ann Arbor Science Publishers, Inc., 1978), pp. 291-305.

6. Thatcher, T. 0. "The Relative Sensitivity of Pacific Northwest Fishes and Invertebrates to Chlorinated Sea Water," in Water Chlorination--Environmental Impact and Health Effects, $\overline{V_{0} 1 .} 2$, R. L. Jolley, Ed. (Ann Arbor, Michigan: Ann Arbor Science Publishers, Inc., 1978), pp. 341-350.

7. Jolley, R. L., G. Jones, W. W. Pitt and J. E. Thompson. "Chlorination of organics in cooling waters and process effluents," in Water Chlorination: Environmental Impact and Health Effects, Vol. l, R. L. Jolley, Ed. (Ann Arbor, Michigan: Ann Arbor Science Publishers, Inc., 1978), pp. 105-138.

8. Bean, R. M., R. G. Riley and P. W. Ryan. "Investigation of halogenated components formed from chlorination of marine water," in Water Chlorination--Environmenta] Impact and Health Effects, Vol. 2, R. L. Jolley, H. Gorchev, D. H. Hamilton, Jr., Eds. (Ann Arbor, Michigan: Ann Arbor Science Publishers, Inc., 1978), pp.223-234.

9. Carpenter, J. H. and C. A. Smith. "Reactions in Chlorinated sea water," in water Chlorination--Environmental Impact and Health Effects, Vol. 2, R. L. Jolley, H. Gorchev, D. H. Hamilton, Jr., Eds. (Ann Arbor, Michigan: Ann Arbor Science Publishers, 1978), pp. 195-208.

10. American Public Health Association. Standard Methods for the Examination of Water and Wastewater, 13th ed. (New York: APHA, 1971), pp. $834-845$. 
Table 1. Initial clam length in exposure tank receiving $100 \%$ control sea water. Date: $2 / 1 / 77$

\begin{tabular}{|c|c|c|c|}
\hline Cles: & Length (inches) & Cian & Length (inches) \\
\hline 1 & 2. 216 & 26 & 1.391 \\
\hline 2 & 1. 886 & 27 & 1. 501 \\
\hline 3 & 1.791 & 23 & 1.564 \\
\hline 4 & 1.469 & 29 & 1.774 \\
\hline 5 & 1.724 & 30 & 1.662 \\
\hline 6 & 1.958 & 31 & 1.598 \\
\hline 7 & 1.659 & 32 & 1.785 \\
\hline B & 2.045 & 33 & 1. 804 \\
\hline 9 & 1.413 & 34 & 1. 896 \\
\hline 10 & 1.503 & 35 & 1.604 \\
\hline 11 & 1.943 & 36 & 1.643 \\
\hline 12 & 1.558 & 37 & 2.038 \\
\hline 13 & 1.660 & 38 & 1. 911 \\
\hline 24 & 1.942 & 39 & 2.061 \\
\hline 15 & 2.298 & 40 & 2.142 \\
\hline 16 & 1.715 & 41 & 1.892 \\
\hline 17 & 1.857 & 42 & 2.111 \\
\hline 18 & 1.876 & 43 & 1.866 \\
\hline 19 & 1.524 & 44 & 1.853 \\
\hline 20 & 1.681 & 45 & 2.031 \\
\hline 21 & 1.338 & 46 & 1. 826 \\
\hline 22 & 1.403 & 47 & 1. 882 \\
\hline 23 & 1.853 & 48 & 3.561 \\
\hline 24 & i. 328 & 49 & 1.797 \\
\hline 25 & 1.462 & 50 & 1. 861 \\
\hline
\end{tabular}

Table 2. Initial clam length in exposure tank receiving $94 \%$ control sea water, $6 \%$ chlorinated sea water. Date: $2 / 1 / 77$

\begin{tabular}{rlrl}
\hline Clan & Length (inches) & clan & Length (inches) \\
\hline 1 & 1.244 & 26 & 1.829 \\
2 & 1.302 & 27 & 2.935 \\
3 & 1.354 & 28 & 2.027 \\
4 & 1.366 & 29 & 2.078 \\
5 & 1.391 & 30 & 2.031 \\
6 & 1.437 & 31 & 1.794 \\
7 & 1.402 & 32 & 1.921 \\
8 & 1.412 & 33 & 1.831 \\
9 & 1.504 & 34 & 2.059 \\
10 & 1.462 & 35 & 1.903 \\
11 & 2.496 & 36 & 1.868 \\
12 & 1.540 & 37 & 1.980 \\
13 & 1.659 & 38 & 2.045 \\
14 & 1.647 & 39 & 2.049 \\
15 & 1.694 & 40 & 2.131 \\
16 & 1.685 & 41 & 1.909 \\
17 & 1.745 & 42 & 2.028 \\
18 & 1.710 & 43 & 2.100 \\
19 & 1.845 & 44 & 2.089 \\
20 & 1.832 & 45 & 2.188 \\
21 & 1.852 & 46 & 2.208 \\
22 & 1.869 & 47 & 2.138 \\
23 & 1.946 & 48 & 2.176 \\
24 & 1.897 & 49 & 1.566 \\
25 & 1.842 & 50 & \\
& & & \\
\hline & & & \\
\hline
\end{tabular}


Table 3. Initial clam length in exposure tank receiving $88 \%$ control sea water, $12 \%$ chlorinated sea water.

Date: $2 / 2 / 77$

\begin{tabular}{llll}
\hline Clam & Length (inches) & cian & Length (inches) \\
\hline 1 & 1.142 & 26 & 1.875 \\
2 & 1.212 & 27 & 1.919 \\
3 & 1.235 & 28 & 1.802 \\
4 & 1.166 & 29 & 1.945 \\
5 & 1.208 & 30 & 1.997 \\
6 & 1.317 & 31 & 2.065 \\
7 & 1.345 & 32 & 2.065 \\
8 & 1.341 & 33 & 1.945 \\
9 & 1.309 & 34 & 2.039 \\
10 & 1.456 & 35 & 2.106 \\
11 & 1.416 & 36 & 2.020 \\
12 & 1.414 & 37 & 2.102 \\
13 & 1.463 & 38 & 1.992 \\
14 & 1.492 & 39 & 1.905 \\
15 & 1.508 & 40 & 2.011 \\
16 & 1.634 & 41 & 2.068 \\
17 & 1.586 & 42 & 2.112 \\
18 & 1.585 & 43 & 2.107 \\
19 & 1.530 & 44 & 2.089 \\
20 & 1.673 & 45 & 2.180 \\
21 & 1.543 & 46 & 2.144 \\
22 & 1.744 & 47 & 1.848 \\
23 & 1.696 & 48 & 2.220 \\
24 & 1.774 & 49 & 2.309 \\
25 & 1.794 & 50 & 2.259 \\
\hline
\end{tabular}

Table 4. Initial clam length in exposure tank receiving $75 \%$ control sea water, $25 \%$ chlorinated sea water. Date: $2 / 2 / 77$

\begin{tabular}{llll}
\hline cla & Length (inches) & clan & Length (inches) \\
\hline 1 & 1.005 & 26 & 1.994 \\
2 & 1.044 & 27 & 1.918 \\
3 & 1.093 & 28 & 1.904 \\
4 & 1.093 & 29 & 2.040 \\
5 & 1.125 & 30 & 1.986 \\
6 & 1.144 & 31 & 1.807 \\
7 & 1.331 & 32 & 1.905 \\
8 & 1.396 & 33 & 1.999 \\
9 & 1.345 & 34 & 2.074 \\
10 & 1.399 & 35 & 2.007 \\
11 & 1.492 & 36 & 2.020 \\
12 & 1.429 & 37 & 2.007 \\
13 & 1.498 & 38 & 2.075 \\
14 & 1.581 & 39 & 2.209 \\
15 & 3.572 & 40 & 2.053 \\
16 & 1.571 & 41 & 2.001 \\
17 & 1.545 & 42 & 1.977 \\
18 & 1.562 & 43 & 2.105 \\
19 & 1.615 & 44 & 2.095 \\
20 & 1.469 & 45 & 2.063 \\
21 & 1.604 & 46 & 2.303 \\
22 & 1.769 & 47 & 2.201 \\
23 & 1.736 & 48 & 2.206 \\
24 & 1.817 & 49 & 2.202 \\
25 & 1.850 & 50 & 1.500 \\
\hline
\end{tabular}


Table 5. Initial clam length in exposure tank receiving 50\% control sea water, $50 \%$ chlorinated sea water. Date: $2 / 2 / 77$

\begin{tabular}{|c|c|c|c|}
\hline clas * & Lengtr (incties) & clan & Length (inches) \\
\hline $\begin{array}{r}1 \\
2 \\
3 \\
4 \\
5 \\
6 \\
7 \\
6 \\
9 \\
10 \\
11 \\
12 \\
13 \\
14 \\
15 \\
16 \\
17 \\
18 \\
19 \\
20 \\
21 \\
22 \\
23 \\
24 \\
25\end{array}$ & $\begin{array}{l}1.249 \\
1.337 \\
1.337 \\
1.398 \\
1.412 \\
1.490 \\
1.568 \\
1.565 \\
1.596 \\
1.563 \\
1.518 \\
1.504 \\
1.610 \\
1.580 \\
1.675 \\
1.619 \\
1.638 \\
1.589 \\
1.596 \\
1.723 \\
1.696 \\
1.708 \\
1.788 \\
1.854 \\
1.780\end{array}$ & $\begin{array}{l}26 \\
27 \\
28 \\
29 \\
30 \\
31 \\
32 \\
33 \\
34 \\
35 \\
36 \\
37 \\
38 \\
39 \\
40 \\
41 \\
42 \\
43 \\
44 \\
45 \\
46 \\
47 \\
49 \\
49 \\
50\end{array}$ & $\begin{array}{r}1.831 \\
1.909 \\
1.888 \\
1.893 \\
1.899 \\
1.910 \\
1.964 \\
1.844 \\
2.125 \\
1.967 \\
1.951 \\
2.048 \\
2.079 \\
2.000 \\
2.106 \\
2.105 \\
2.079 \\
2.188 \\
2.195 \\
.2 .153 \\
2.127 \\
2.289 \\
2.105 \\
2.233 \\
2.189\end{array}$ \\
\hline
\end{tabular}

Table 6. Initial clam length in exposure tank receiving $100 \%$ chlorinated sea water. Date: $2 / 3 / 77$




Table 7. Length of clams in tank receiving $100 \%$ control sea water after 1 month of exposure. Date: $3 / 1 / 77$

\begin{tabular}{llll}
\hline Cian & Length (inches) & Clan & Length (inches) \\
\hline 1 & 2.215 & 26 & \\
2 & 1.884 & 27 & 1.387 \\
3 & 1.787 & 28 & 1.566 \\
4 & 1.468 & 29 & 1.770 \\
5 & 1.723 & 30 & 1.656 \\
6 & 1.952 & 31 & 1.593 \\
7 & 1.654 & 32 & 1.784 \\
8 & 2.042 & 33 & 1.800 \\
9 & 1.407 & 34 & 1.915 \\
10 & 1.500 & 35 & 1.590 \\
11 & 1.933 & 36 & 1.636 \\
12 & 1.555 & 37 & 2.030 \\
13 & 1.658 & 38 & 1.914 \\
14 & 1.937 & 39 & 2.054 \\
15 & 2.295 & 40 & 2.140 \\
16 & 1.716 & 41 & 1.889 \\
17 & 1.850 & 42 & 2.110 \\
18 & 1.675 & 43 & 1.857 \\
19 & 1.522 & 44 & 1.850 \\
20 & 1.678 & 45 & 2.027 \\
21 & 1.333 & 46 & 1.822 \\
22 & 1.400 & 47 & 1.877 \\
23 & 1.651 & 48 & 1.561 \\
24 & 1.326 & 49 & 1.796 \\
25 & 1.461 & 50 & 1.857 \\
\hline
\end{tabular}

Table 8 . Length of clams in tank receiving $94 \%$ control sea water, $6 \%$ chlorinated sea water after 1 month of exposure. Date: $3 / 1 / 77$

\begin{tabular}{llll}
\hline Clam ingth (inches) & Clan & Length (inches) \\
\hline 1 & 1.242 & & 1.825 \\
2 & 1.300 & 26 & 2.829 \\
3 & 1.352 & 28 & 2.019 \\
4 & 1.362 & 29 & 2.073 \\
5 & 1.389 & 30 & 2.080 \\
6 & 1.432 & 31 & 1.791 \\
7 & 1.400 & 32 & 1.922 \\
8 & 1.412 & 33 & 1.835 \\
9 & 1.501 & 34 & 2.055 \\
10 & 1.480 & 35 & 1.900 \\
11 & 1.494 & 36 & 1.863 \\
12 & 1.539 & 37 & 1.977 \\
13 & 1.657 & 38 & 1.851 \\
14 & 1.643 & 39 & 2.036 \\
15 & 1.689 & 40 & 2.048 \\
16 & 1.684 & 41 & 2.124 \\
17 & 1.741 & 42 & 1.903 \\
18 & 1.712 & 43 & 2.035 \\
19 & 1.839 & 44 & 2.097 \\
20 & 1.830 & 45 & 2.086 \\
21 & 1.890 & 46 & 2.185 \\
22 & 1.866 & 47 & 2.208 \\
23 & 1.944 & 48 & 2.134 \\
24 & 1.890 & 49 & 2.173 \\
25 & 1.836 & 50 & 1.586 \\
\hline
\end{tabular}


Table 9. Length of clams in tank recejving $88 \%$ control sea water, $12 \%$ chlorinated sea water after 1 month of exposure. Date: $3 / 2 / 77$

\begin{tabular}{|c|c|c|c|}
\hline Cla & Length (inches) & clat & Length (inches) \\
\hline $\begin{array}{r}1 \\
2 \\
3 \\
4 \\
5 \\
6 \\
7 \\
8 \\
9 \\
10 \\
11 \\
12 \\
13 \\
14 \\
15 \\
16 \\
17 \\
18 \\
19 \\
20 \\
21 \\
22 \\
23 \\
24 \\
25\end{array}$ & $\begin{array}{l}1.139 \\
1.216 \\
1.234 \\
1.163 \\
1.206 \\
1.315 \\
1.342 \\
1.338 \\
1.307 \\
1.453 \\
1.412 \\
1.411 \\
1.460 \\
1.490 \\
1.507 \\
1.632 \\
1.583 \\
1.586 \\
1.532 \\
1.670 \\
1.540 \\
1.742 \\
1.694 \\
1.772 \\
1.793\end{array}$ & $\begin{array}{l}26 \\
27 \\
28 \\
29 \\
30 \\
31 \\
32 \\
33 \\
34 \\
35 \\
36 \\
37 \\
38 \\
39 \\
40 \\
41 \\
42 \\
43 \\
44 \\
45 \\
46 \\
47 \\
48 \\
49 \\
50\end{array}$ & $\begin{array}{l}1.870 \\
1.916 \\
1.800 \\
1.939 \\
1.996 \\
2.061 \\
2.062 \\
1.943 \\
2.037 \\
2.100 \\
2.018 \\
2.100 \\
1.998 \\
1.900 \\
2.013 \\
2.064 \\
2.109 \\
2.108 \\
2.095 \\
2.178 \\
2.143 \\
1.846 \\
2.218 \\
2.308 \\
2.257\end{array}$ \\
\hline
\end{tabular}

Table 10. Length of clams in tank receiving $75 \%$ control sea water, 25\% chlorinated sea water after 1 month of exposure. Date: $3 / 4 / 77$

\begin{tabular}{|c|c|c|c|}
\hline Clan & Length (inches) & Cla & Length (inches) \\
\hline $\begin{array}{r}1 \\
2 \\
3 \\
4 \\
5 \\
6 \\
7 \\
8 \\
9 \\
10 \\
11 \\
12 \\
13 \\
14 \\
15 \\
16 \\
17 \\
18 \\
19 \\
20 \\
21 \\
22 \\
23 \\
24 \\
25\end{array}$ & $\begin{array}{l}1.003 \\
1.042 \\
1.090 \\
1.092 \\
1.123 \\
1.141 \\
1.330 \\
1.382 \\
1.343 \\
1.396 \\
1.490 \\
1.422 \\
1.495 \\
1.576 \\
1.576 \\
1.568 \\
1.543 \\
1.562 \\
1.610 \\
2.230 \\
1.600 \\
1.760 \\
1.730 \\
1.812 \\
1.846\end{array}$ & $\begin{array}{l}25 \\
27 \\
28 \\
29 \\
30 \\
31 \\
32 \\
33 \\
34 \\
35 \\
36 \\
37 \\
38 \\
39 \\
40 \\
41 \\
42 \\
43 \\
44 \\
45 \\
46 \\
47 \\
48 \\
49 \\
50\end{array}$ & $\begin{array}{l}1.893 \\
1.918 \\
1.904 \\
2.036 \\
1.995 \\
1.805 \\
1.904 \\
1.995 \\
2.072 \\
2.005 \\
2.015 \\
2.004 \\
2.073 \\
2.203 \\
2.049 \\
1.999 \\
1.969 \\
2.113 \\
2.095 \\
2.065 \\
2.305 \\
2.206 \\
2.205 \\
2.200 \\
1.498\end{array}$ \\
\hline
\end{tabular}


Table 11. Length of clams in tank receiving $50 \%$ control sea water, $50 \%$ chlorinated sea water after 1 month of exposure. Date: $3 / 4 / 77$

\begin{tabular}{llll}
\hline Clan & Lengeh (inches) & Cl & Length (inches) \\
\hline 1 & 1.250 & 26 & 1.828 \\
2 & 1.335 & 27 & 1.908 \\
3 & 1.336 & 28 & 1.889 \\
4 & 1.398 & 29 & 1.898 \\
5 & 1.410 & 30 & 1.892 \\
6 & 1.486 & 31 & 1.909 \\
7 & 1.569 & 32 & 1.962 \\
8 & 1.563 & 33 & 1.842 \\
9 & 1.596 & 34 & 2.124 \\
10 & 1.561 & 35 & 1.965 \\
11 & 1.517 & 36 & 1.949 \\
12 & 1.500 & 37 & 2.042 \\
13 & 1.610 & 38 & 2.076 \\
14 & 1.579 & 39 & 1.999 \\
15 & 1.673 & 40 & 2.100 \\
16 & 1.618 & 41 & 2.105 \\
17 & 1.636 & 42 & 2.079 \\
18 & 1.588 & 43 & 2.183 \\
19 & 1.595 & 44 & 2.196 \\
20 & 1.722 & 45 & 2.153 \\
21 & 1.695 & 46 & 2.128 \\
22 & 1.707 & 47 & 2.287 \\
23 & 1.788 & 48 & 2.105 \\
24 & 1.859 & 49 & 2.233 \\
25 & 1.778 & 50 & 2.186 \\
\hline
\end{tabular}

Table 12. Length of clams in tank receiving $100 \%$ chlorinated sea water after 1 month of expsoure. Date: 3/4/77

\begin{tabular}{|c|c|c|c|}
\hline Clan & Lendth (inches) & Clas & Lenoth (inches) \\
\hline $\begin{array}{l}2 \\
2 \\
3 \\
4 \\
5 \\
6 \\
7 \\
8 \\
9 \\
10 \\
11 \\
12 \\
13 \\
14 \\
15 \\
16 \\
17 \\
18 \\
19 \\
20 \\
21 \\
22 \\
23 \\
24 \\
25\end{array}$ & $\begin{array}{l}1.118 \\
1.240 \\
1.324 \\
1.296 \\
1.356 \\
1.400 \\
1.565 \\
1.628 \\
1.598 \\
1.584 \\
1.380 \\
1.593 \\
1.625 \\
1.607 \\
1.663 \\
1.638 \\
1.644 \\
1.670 \\
1.658 \\
1.658 \\
1.690 \\
1.713 \\
1.694 \\
1.837 \\
1.786\end{array}$ & $\begin{array}{l}26 \\
27 \\
28 \\
29 \\
30 \\
31 \\
32 \\
33 \\
34 \\
35 \\
36 \\
37 \\
38 \\
39 \\
40 \\
41 \\
42 \\
43 \\
44 \\
45 \\
46 \\
47 \\
48 \\
49 \\
50\end{array}$ & $\begin{array}{l}1.836 \\
1.798 \\
1.738 \\
1.957 \\
1.964 \\
1.876 \\
1.844 \\
1.933 \\
1.967 \\
1.960 \\
1.985 \\
2.069 \\
2.100 \\
2.153 \\
2.120 \\
1.950 \\
2.168 \\
2.003 \\
2.043 \\
2.104 \\
2.175 \\
2.202 \\
2.111 \\
2.055 \\
2.175\end{array}$ \\
\hline
\end{tabular}


Table 13. Length of clams added to tanks to replace animals removed for chemical and histological examination.

\begin{tabular}{|c|c|c|c|c|c|c|}
\hline \multirow[b]{2}{*}{ CONTROL } & \multirow[t]{2}{*}{ Length (inches) } & \multicolumn{2}{|c|}{ Length (incties) } & \multicolumn{3}{|c|}{ Length (inches) } \\
\hline & & \multicolumn{2}{|c|}{ 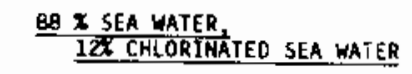 } & \multicolumn{3}{|c|}{$\frac{50 X \text { SEA WATER, }}{\text { 50\% CHLORINATED SEA WATE }}$} \\
\hline $\begin{array}{l}51 \\
52 \\
53 \\
54 \\
55 \\
56 \\
57 \\
58 \\
59 \\
60\end{array}$ & $\begin{array}{l}1.923 \\
1.979 \\
1.893 \\
1.968 \\
1.788 \\
1.770 \\
1.867 \\
1.884 \\
2.056 \\
1.912\end{array}$ & $\begin{array}{l}51 \\
52 \\
53 \\
54 \\
55 \\
56 \\
57 \\
58 \\
59 \\
60\end{array}$ & $\begin{array}{l}1.880 \\
1.868 \\
1.878 \\
2.008 \\
1.824 \\
1.659 \\
1.915 \\
1.921 \\
1.909 \\
1.710\end{array}$ & $\begin{array}{l}51 \\
52 \\
53 \\
54 \\
55 \\
56 \\
57 \\
58 \\
59 \\
60\end{array}$ & & $\begin{array}{l}1.764 \\
1.903 \\
1.845 \\
1.736 \\
1.771 \\
1.834 \\
1.745 \\
1.767 \\
1.781 \\
2.071\end{array}$ \\
\hline$\frac{94 X \text { SEA WATER, }}{\text { 6X CHLORINATED }}$ & SEA WATER & $\frac{75 X \text { SEA WATER, }}{\text { 25X CHLORINATED }}$ & SEA WATER & $100 x$ & CHLORINATED & SEA WATER \\
\hline $\begin{array}{l}51 \\
52 \\
53 \\
54 \\
55 \\
56 \\
57 \\
58 \\
59 \\
60\end{array}$ & $\begin{array}{l}1.916 \\
1.919 \\
1.893 \\
1.879 \\
1.709 \\
1.813 \\
1.610 \\
1.722 \\
1.950 \\
1.724\end{array}$ & $\begin{array}{l}51 \\
52 \\
53 \\
54 \\
55 \\
56 \\
57 \\
58 \\
59 \\
60\end{array}$ & $\begin{array}{l}1.699 \\
1.890 \\
1.892 \\
1.692 \\
1.902 \\
1.911 \\
1.789 \\
1.921 \\
2.054 \\
2.011\end{array}$ & $\begin{array}{l}51 \\
52 \\
53 \\
54 \\
55 \\
56 \\
57 \\
58 \\
59 \\
60 \\
61 \\
62\end{array}$ & & $\begin{array}{l}1.664 \\
1.556 \\
1.767 \\
1.783 \\
1.659 \\
1.544 \\
1.607 \\
1.771 \\
1.879 \\
2.004 \\
1.529 \\
1.875\end{array}$ \\
\hline
\end{tabular}

Table 14. Length of clams in tank receiving $100 \%$ control sea water after 2 months of exposure. Date: 4/4/77

\begin{tabular}{|c|c|c|c|}
\hline Clan & Length (inches) & $\mathrm{Clan}$ & Length (inches) \\
\hline $\begin{array}{r}1 \\
2 \\
3 \\
4 \\
5 \\
6 \\
7 \\
8 \\
9 \\
10 \\
11 \\
12 \\
13 \\
14 \\
15 \\
16 \\
17 \\
18 \\
19 \\
20 \\
21 \\
22 \\
23 \\
24 \\
25 \\
26 \\
27 \\
28 \\
29 \\
30\end{array}$ & $\begin{array}{l}2.215 \\
1.824 \\
\text { Removed* } \\
1.465 \\
\text { Removed* } \\
1.951 \\
\text { Removed* } \\
\text { Removed* } \\
1.406 \\
1.501 \\
1.932 \\
1.556 \\
1.656 \\
1.936 \\
2.294 \\
1.713 \\
1.849 \\
1.674 \\
1.521 \\
\text { Removed* } \\
1.332 \\
1.400 \\
1.650 \\
1.326 \\
1.460 \\
1.386 \\
\text { Removed } \\
1.562 \\
1.770 \\
1.654\end{array}$ & $\begin{array}{l}31 \\
32 \\
33 \\
34 \\
35 \\
36 \\
37 \\
38 \\
39 \\
40 \\
41 \\
42 \\
43 \\
44 \\
45 \\
46 \\
47 \\
48 \\
49 \\
50 \\
51 \\
52 \\
53 \\
54 \\
55 \\
56 \\
57 \\
58 \\
59 \\
60\end{array}$ & $\begin{array}{l}1.592 \\
1.781 \\
1.800 \\
1.913 \\
1.598 \\
\text { Removed* } \\
2.029 \\
1.912 \\
\text { Removed* } \\
2.140 \\
1.889 \\
2.108 \\
1.857 \\
1.849 \\
\text { Removed } \\
1.822 \\
\text { Removed } \\
1.561 \\
1.796 \\
1.857 \\
1.922 \\
1.977 \\
1.890 \\
1.965 \\
1.787 \\
1.768 \\
1.866 \\
1.893 \\
2.054 \\
1.911\end{array}$ \\
\hline
\end{tabular}

* Removed for Chemical or Histological Analysis. 
Table 15. Length of clams in tank receiving $94 \%$ control sea water, $6 \%$ chlorinated sea water after 2 months of exposure. Date: $4 / 5 / 77$

\begin{tabular}{|c|c|c|c|c|c|}
\hline $\mathrm{Clan}$ & Length (inches) & $\mathrm{Clan}$. & Length (inches) & Clam & Length (inches) \\
\hline 1 & 1.242 & 21 & Removed* & 41 & 2.123 \\
\hline 2 & 1. 298 & 22 & 1.867 & 42 & 1. 903 \\
\hline 3 & 1. 352 & 23 & 1. 943 & 43 & 2.034 \\
\hline 4 & 1. 361 & 24 & 1.891 & 44 & 2. 098 \\
\hline 5 & 1. 389 & 25 & Removed & 45 & Removed* \\
\hline 6 & 1.430 & 26 & 1. 825 & 46 & 2.185 \\
\hline 7 & 1. 401 & 27 & 1. 829 & 47 & Removed \\
\hline 8 & 1.411 & 28 & Removed & 48 & Removed* \\
\hline 9 & 1.501 & 29 & 2.072 & 49 & Removed $^{\star}$ \\
\hline 10 & 1.460 & 30 & 2.081 & 50 & 1.565 \\
\hline 11 & 1.487 & 31 & 1.791 & 51 & 1.916 \\
\hline 12 & Removed* & 32 & 1.925 & 52 & 1.919 \\
\hline 13 & 1.656 & 33 & 1. 834 & 53 & 1. 892 \\
\hline 14 & 1. 543 & 34 & 2.057 & 54 & 1. 879 \\
\hline 15 & 1.689 & 35 & 1.898 & 55 & 1.709 \\
\hline 16 & 1. 685 & 36 & 1. 874 & 56 & 1.811 \\
\hline 17 & 1.740 & 37 & 1.977 & 57 & 1.609 \\
\hline 18 & 1.711 & 38 & 1.850 & 58 & 1.722 \\
\hline 19 & Removed* & 39 & 2.035 & 59 & 1.950 \\
\hline 20 & Removed ${ }^{*}$ & 40 & 2.047 & 60 & 1.726 \\
\hline
\end{tabular}

* Rempyed for Chemical or Histological Analysis.

Table 16. Length of clams in tank receiving $88 \%$ control sea water, $12 \%$ chlorinated sea water after 2 months of exposure. Date: $4 / 5 / 77$

\begin{tabular}{|c|c|c|c|}
\hline Clan & Length (inches) & Clan & Length (inches) \\
\hline 1 & 1.139 & 31 & 2.062 \\
\hline 2 & 1.216 & 32 & 2.061 \\
\hline $\mathbf{3}$ & 1. 234 & 33 & 1. 942 \\
\hline 4 & 1. 164 & 34 & 2.034 \\
\hline 5 & 1. 205 & 35 & 2.100 \\
\hline 6 & 1.315 & 36 & 2.013 \\
\hline 7 & Removed ${ }^{*}$ & 37 & Removeded* \\
\hline 8 & 1.337 & 38 & 1. 988 \\
\hline 9 & Renoved* & 39 & 1.901 \\
\hline 10 & 1.452 & 40 & 2.022 \\
\hline 11 & Removed & 41 & 2. 064 \\
\hline 12 & 1.410 & 42 & 2. 108 \\
\hline 13 & 1. 460 & 43 & 2.107 \\
\hline 14 & 1. 490 & 44 & 2. 094 \\
\hline 15 & 1.507 & 45 & Removed* \\
\hline 16 & 1.632 & 46 & Removed" \\
\hline .77 & 1.583 & 47 & 1.846 \\
\hline 18 & Renoved* & 48 & 2. 216 \\
\hline 19 & 1.525 & 49 & 2.309 \\
\hline 20 & 1.670 & so & Removed $^{\star}$ \\
\hline 21 & 1.539 & 51 & 1.880 \\
\hline 22 & 1. 741 & 52 & 1.868 \\
\hline 23 & 1.695 & 53 & 1.876 \\
\hline 24 & Removed* & 54 & 2.000 \\
\hline 25 & Removed* & 55 & 1.823 \\
\hline 26 & 1.870 & 56 & 1.656 \\
\hline 27 & 1.915 & 57 & 1. 904 \\
\hline 28 & 1.800 & 58 & 1. 921 \\
\hline 29 & 1.939 & 59 & 1. 909 \\
\hline 30 & 1.995 & 60 & 1.709 \\
\hline
\end{tabular}

* Removed for Chenical or Histological Analysis. 
Table 17. Length of clams in tank receiving 75\% control sea water, $25 \%$ chlorinated sea water after 2 months of exposure. Date: $4 / 5 / 77$

\begin{tabular}{|c|c|c|c|}
\hline Clan & Length (inches) & $C \cos \theta$ & Length (inches) \\
\hline 1 & 1.003 & 31 & 1. 8004 \\
\hline 2 & 1.040 & 32 & Renoved* \\
\hline 3 & 1.090 & 33 & 1.994 \\
\hline 4 & 1.090 & 34 & 2.071 \\
\hline 5 & 1.123 & 35 & 2.005 \\
\hline 6 & 1.141 & 36 & 2.011 \\
\hline 7 & Removed ${ }^{\star}$ & 37 & Renoved \\
\hline 8 & 1.381 & 38 & 2.073 \\
\hline 9 & 1.344 & 39 & 2.206 \\
\hline 10 & 1.396 & 40 & Removed ${ }^{\star}$ \\
\hline 11 & 1.488 & 41 & 1.999 \\
\hline 12 & 1.422 & 42 & I. 980 \\
\hline 23 & 1.496 & 43 & Removed* \\
\hline 24 & 1.576 & 44 & 2.096 \\
\hline 15 & 1.570 & 45 & 2. 069 \\
\hline 16 & 1.569 & 46 & Removed* \\
\hline 17 & Remaved & 47 & Removed* \\
\hline 18 & 1.562 & 48 & 2.204 \\
\hline 19 & 1.610 & 49 & 2.200 \\
\hline 20 & 2.229 & 50 & 1.500 \\
\hline 21 & 1.600 & 51 & 1.699 \\
\hline 22 & 3.760 & 52 & 1. 890 \\
\hline 23 & 1.734 & 53 & 1. 894 \\
\hline 24 & 1.814 & 54 & 1.692 \\
\hline 25 & Removed" & 55 & 1. 901 \\
\hline 26 & 1.892 & 56 & 1.912 \\
\hline 27 & Removed* & 57 & 1.783 \\
\hline 28 & Removed ${ }^{\star}$ & 58 & 1.921 \\
\hline 29 & 2. 035 & 59 & 2.067 \\
\hline 30 & 1. 985 & 60 & 2.010 \\
\hline
\end{tabular}

* Removed for Chemical or Histological Analysis.

Table 18. Length of clams in tank receiving $50 \%$ control sea water, $50 \%$ chlorinated sea water after 2 months of exposure. Date: $4 / 6 / 77$

\begin{tabular}{|c|c|c|c|}
\hline clan & Length (inches) & clan & Length (inches) \\
\hline $\begin{array}{r}1 \\
2 \\
3 \\
4 \\
5 \\
6 \\
7 \\
8 \\
9 \\
10 \\
11 \\
12 \\
13 \\
14 \\
15 \\
16 \\
17 \\
18 \\
19 \\
20 \\
21 \\
22 \\
23 \\
24 \\
25 \\
26 \\
27 \\
28 \\
29 \\
30\end{array}$ & $\begin{array}{l}1.246 \\
1.335 \\
\text { Removed } \\
1.392 \\
1.408 \\
1.486 \\
\text { Removed* } \\
1.562 \\
\text { Remuved* } \\
1.559 \\
1.514 \\
1.499 \\
1.609 \\
1.575 \\
1.671 \\
1.612 \\
1.634 \\
1.587 \\
\text { Removed* } \\
1.722 \\
1.692 \\
1.705 \\
1.786 \\
1.852 \\
1.778 \\
1.827 \\
1.907 \\
1.886 \\
\text { Removed } \\
1.890\end{array}$ & $\begin{array}{l}31 \\
32 \\
33 \\
34 \\
35 \\
36 \\
37 \\
38 \\
39 \\
40 \\
41 \\
42 \\
43 \\
44 \\
45 \\
46 \\
47 \\
48 \\
49 \\
50 \\
51 \\
52 \\
53 \\
54 \\
55 \\
56 \\
57 \\
58 \\
59 \\
60\end{array}$ & $\begin{array}{l}\text { 1. } 908 \\
1.960 \\
1.841 \\
2.121 \\
1.968 \\
\text { Removed* } \\
\text { Removed* } \\
\text { Removed* } \\
\text { Removed } \\
2.100 \\
2.102 \\
2.077 \\
2.180 \\
2.191 \\
2.152 \\
2.125 \\
2.286 \\
2.110 \\
\text { Removed* } \\
2.190 \\
1.763 \\
1.902 \\
1.844 \\
1.735 \\
1.770 \\
\text { Removed } 3 / 30 / 77 \\
1.743 \\
1.767 \\
1.780 \\
2.065\end{array}$ \\
\hline
\end{tabular}

* Removed for Chemical or Histological Analysis. 
Table 19. Length of clams in tank receiving $100 \%$ chlorinated sea water after 2 months of exposure. Date: 4/6/77

\begin{tabular}{|c|c|c|c|}
\hline C1 am * & length (incres) & Cian & Length (inches) \\
\hline $\begin{array}{r}1 \\
2 \\
3 \\
4 \\
5 \\
6 \\
7 \\
8 \\
9 \\
10 \\
21 \\
12 \\
13 \\
14 \\
15 \\
16 \\
17 \\
18 \\
19 \\
20 \\
21 \\
22 \\
23 \\
24 \\
25 \\
26 \\
27 \\
28 \\
29 \\
30\end{array}$ & $\begin{array}{l}\text { 1. } 116 \\
\text { Removed } \\
1.315 \\
1.295 \\
1.355 \\
1.400 \\
1.566 \\
1.627 \\
1.597 \\
1.582 \\
\text { Removed } \\
\text { Removed } \\
1.625 \\
1.604 \\
1.652 \\
1.637 \\
1.644 \\
1.673 \\
\text { Removed } \\
1.656 \\
1.690 \\
\text { Removed } \\
1.694 \\
1 . \text { B36 } \\
1.789 \\
\text { Removed }^{\star} \\
1.799 \\
\text { Removed }^{\star} \\
1.856 \\
1.963 \\
\end{array}$ & $\begin{array}{l}31 \\
32 \\
33 \\
34 \\
35 \\
36 \\
37 \\
38 \\
39 \\
40 \\
41 \\
42 \\
43 \\
44 \\
45 \\
46 \\
47 \\
48 \\
49 \\
50 \\
51 \\
52 \\
53 \\
54 \\
55 \\
56 \\
57 \\
58 \\
59 \\
60\end{array}$ & $\begin{array}{l}1.875 \\
\text { Remaved* } \\
1.936 \\
1.966 \\
1.963 \\
1.964 \\
\text { Removed* } \\
2.097 \\
2.153 \\
2.117 \\
\text { Removed* } \\
\text { Removed } \\
\text { Removed* } \\
2.042 \\
2.104 \\
2.170 \\
2.002 \\
2.115 \\
2.055 \\
2.173 \\
1.664 \\
1.555 \\
1.764 \\
1.782 \\
1.660 \\
1.562 \\
1.605 \\
1.770 \\
1.879 \\
2.005\end{array}$ \\
\hline
\end{tabular}

* Removed for Chemical or Histological Analysis.

Table 20. Length of clams in tank receiving $100 \%$ control sea water after 3 months of exposure. Date: $5 / 2 / 77$

\begin{tabular}{|c|c|c|c|c|c|}
\hline $\mathrm{Clan}$ & Length (inches) & Cian & Length (inches) & clam * & length (Inches) \\
\hline $\begin{array}{l}1 \\
2 \\
3 \\
4 \\
5 \\
6 \\
7 \\
8 \\
9 \\
10 \\
11 \\
12 \\
13 \\
14 \\
15 \\
16 \\
17 \\
18 \\
19 \\
20 \\
21 \\
22 \\
23 \\
24 \\
25 \\
26 \\
27\end{array}$ & 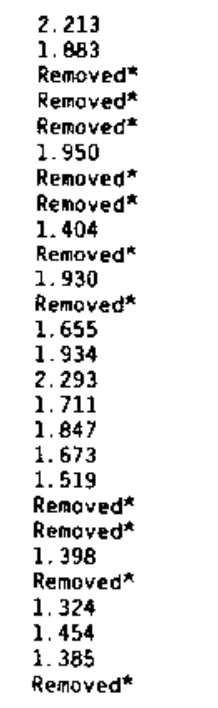 & $\begin{array}{l}28 \\
29 \\
30 \\
31 \\
32 \\
33 \\
34 \\
35 \\
36 \\
37 \\
38 \\
39 \\
40 \\
41 \\
42 \\
43 \\
44 \\
45 \\
46 \\
47 \\
48 \\
49 \\
50 \\
51 \\
52 \\
53 \\
54\end{array}$ & 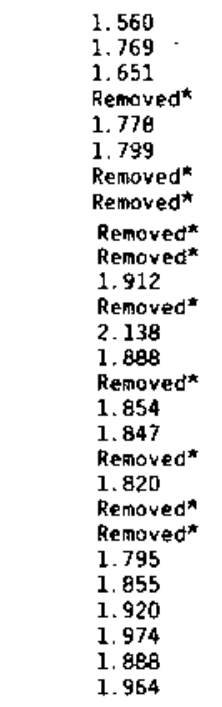 & $\begin{array}{l}55 \\
56 \\
57 \\
58 \\
59 \\
60 \\
61 \\
71 \\
72 \\
73 \\
74 \\
75 \\
76 \\
77 \\
78 \\
79 \\
80\end{array}$ & $\begin{array}{l}1.784 \\
1.765 \\
1.863 \\
1.879 \\
2.051 \\
1.910 \\
1.432 \\
1.279 \\
1.175 \\
1.209 \\
1.292 \\
1.359 \\
1.177 \\
1.288 \\
1.396 \\
1.265 \\
1.200\end{array}$ \\
\hline
\end{tabular}

* Removed for Chemical or Histological Analysis. 
Table 21 . Length of clams in tank receiving $94 \%$ control sea water, $6 \%$ chlorinated sea water after 3 months of exposure. Date: $5 / 3 / 77$

\begin{tabular}{|c|c|c|c|}
\hline Clat & Length (inches) & $\cos { }^{\circ}$ & Length (inches) \\
\hline 1 & 1.240 & 36 & 1. 860 \\
\hline 2 & 1. 297 & 37 & 1.974 \\
\hline 3 & 1. 349 & 38 & Removed \\
\hline 4 & Removed" & 39 & 2.034 \\
\hline 5 & Removed ${ }^{\star}$ & 40 & 2.045 \\
\hline 6 & 1.428 & 41 & 2.122 \\
\hline 7 & 1.399 & 42 & Renoved ${ }^{k}$ \\
\hline 8 & 1.409 & 43 & Remaved* \\
\hline 9 & 1.499 & 44 & 2.094 \\
\hline 10 & 1.458 & 45 & Removed ${ }^{*}$ \\
\hline 11 & Removed* & 46 & 2.185 \\
\hline 12 & Removed* & 47 & Removed* \\
\hline 13 & 1.654 & 48 & Remored* \\
\hline 14 & Removed* & 49 & Removed ${ }^{\star}$ \\
\hline 15 & 1.687 & 50 & 1.563 \\
\hline 16 & 1.683 & 51 & 1.913 \\
\hline 17 & 1.739 & 52 & 1.916 \\
\hline 18 & 1.710 & 53 & 1. 889 \\
\hline 19 & Removed ${ }^{\star}$ & 54 & 1.876 \\
\hline 20 & Removed ${ }^{\star}$ & 55 & 1.707 \\
\hline 21 & Removed ${ }^{\star}$ & 56 & 1. 800 \\
\hline 22 & 1.865 & 57 & 1.607 \\
\hline 23 & 1.942 & 58 & 1.717 \\
\hline 24 & Removed" & 59 & 1.948 \\
\hline 25 & Removed ${ }^{*}$ & 60 & 1.722 \\
\hline 26 & 1. 823 & 71 & 1.430 \\
\hline 27 & 3.825 & 72 & 1. 390 \\
\hline 28 & Remaved* & 73 & 1. 339 \\
\hline 29 & Remaved ${ }^{*}$ & 74 & 1.216 \\
\hline 30 & 2.029 & 75 & 1.173 \\
\hline 31 & Removed & 76 & 1.338 \\
\hline 32 & 1.923 & 77 & 1.529 \\
\hline 33 & 1.831 & 78 & 1.416 \\
\hline 34 & 2.053 & 79 & 1. 158 \\
\hline 35 & 1.897 & 80 & 1.314 \\
\hline
\end{tabular}

* Removed for Chemical or Hiștological Analysis.

Table 22. Length of clams in tank receiving $88 \%$ control sea water, $12 \%$ chlorinated sea water after 3 months of exposure. Date: $5 / 4 / 77$

\begin{tabular}{|c|c|c|c|c|c|}
\hline Clan & Length (inches) & Clan & Length (inches) & Clan & Length (inches) \\
\hline $\begin{array}{r}1 \\
2 \\
3 \\
4 \\
5 \\
6 \\
7 \\
8 \\
9 \\
10 \\
11 \\
12 \\
13 \\
14 \\
15 \\
16 \\
17 \\
18 \\
19 \\
20 \\
21 \\
22 \\
23 \\
24 \\
25 \\
26\end{array}$ & $\begin{array}{l}\text { 1. } 137 \\
\text { Removed" } \\
1.232 \\
1.160 \\
\text { Removed* } \\
1.314 \\
\text { Removed* } \\
1.335 \\
\text { Removed* } \\
1.450 \\
\text { Removed* } \\
1.408 \\
1.457 \\
1.488 \\
1.504 \\
\text { Removed* } \\
1.581 \\
\text { Renoved* } \\
\text { Renoved* } \\
1.669 \\
1.537 \\
\text { Removed* } \\
\text { Removed* } \\
\text { Removed* } \\
\text { Removed* } \\
\text { 1.858 }\end{array}$ & $\begin{array}{l}27 \\
28 \\
29 \\
30 \\
31 \\
32 \\
33 \\
34 \\
35 \\
36 \\
37 \\
38 \\
39 \\
40 \\
41 \\
42 \\
43 \\
44 \\
45 \\
46 \\
47 \\
48 \\
49 \\
50 \\
51 \\
52\end{array}$ & $\begin{array}{l}\text { 1. } 914 \\
1.796 \\
\text { Removed } \\
1.992 \\
2.059 \\
\text { Removed* } \\
\text { Removed* } \\
2.035 \\
2.098 \\
2.015 \\
\text { Removed* } \\
1.985 \\
1.899 \\
2.003 \\
2.063 \\
\text { Removed* } \\
2.108 \\
2.084 \\
\text { Removed } \\
\text { Removed* } \\
\text { Removed } \\
2.215 \\
2.305 \\
\text { Removed } \\
1.878 \\
1.864\end{array}$ & $\begin{array}{l}53 \\
54 \\
55 \\
56 \\
57 \\
58 \\
59 \\
60 \\
61 \\
71 \\
72 \\
73 \\
74 \\
75 \\
76 \\
77 \\
78 \\
79 \\
80\end{array}$ & $\begin{array}{l}1.873 \\
2.008 \\
1.821 \\
1.658 \\
1.913 \\
1.919 \\
1.904 \\
1.706 \\
1.134 \\
1.515 \\
1.219 \\
1.413 \\
1.116 \\
1.381 \\
1.207 \\
1.363 \\
1.418 \\
1.323 \\
1.543\end{array}$ \\
\hline
\end{tabular}

* Removed for Chemical or Histological Analysis. 
Table 23. Length of clams in tank receiving $75 \%$ control sea water, $25 \%$ chlorinated sea water after 3 months of exposure. Date $5 / 4 / 77$

\begin{tabular}{|c|c|c|c|}
\hline Clan & Length (inches) & clam 1 & Length (inches) \\
\hline 1 & 1.000 & 36 & 2.019 \\
\hline 2 & 1.039 & 37 & Removed* \\
\hline 3 & 1.087 & 38 & Removed* \\
\hline 4 & Removed* & 39 & Removed ${ }^{*}$ \\
\hline 5 & 1.120 & 40 & Removed* \\
\hline 6 & Removed* & 41 & 1.998 \\
\hline 7 & Removedx & 42 & Remaved* \\
\hline B & Removed ${ }^{\star}$ & 43 & Removed ${ }^{\star}$ \\
\hline 9 & 1.342 & 44 & 2.093 \\
\hline 10 & 1.395 & 45 & 2.073 \\
\hline 11 & Removed ${ }^{\star}$ & 46 & Remaved \\
\hline 12 & 1.420 & 47 & Removed $^{\star}$ \\
\hline 13 & 1.493 & 48 & Removed ${ }^{*}$ \\
\hline 14 & 1.574 & 49 & Removed* \\
\hline 15 & 1. 568 & 50 & 1.496 \\
\hline 16 & 1. 567 & 51 & 1.696 \\
\hline 17 & Removed ${ }^{\star}$ & 52 & 1.887 \\
\hline 18 & 1.560 & 53 & 1. 892 \\
\hline 19 & Removed* & 54 & 1.692 \\
\hline 20 & Removed" & 55 & 1.896 \\
\hline 21 & Removed & 56 & 1.909 \\
\hline 22 & 1.758 & 57 & 1.780 \\
\hline 23 & 1. 735 & 58 & 1.919 \\
\hline 24 & 1.812 & 59 & 2.068 \\
\hline 25 & Removed* & 60 & 2.017 \\
\hline 26 & 1.889 & 61 & 1.197 \\
\hline 27 & Removed* & 71 & I. 442 \\
\hline 28 & Removed ${ }^{*}$ & 72 & 1. 329 \\
\hline 29 & 2.037 & 73 & 1.215 \\
\hline 30 & 3.983 & 74 & 1.307 \\
\hline 31 & 1.803 & 75 & 1.204 \\
\hline 32 & Removed* & 76 & 1.355 \\
\hline 33 & 1.992 & 77 & 1. 293 \\
\hline 34 & 2.070 & 78 & 1.404 \\
\hline \multirow[t]{2}{*}{35} & 2.004 & 79 & 1. 328 \\
\hline & & BO & 1.128 \\
\hline
\end{tabular}

* Removed for Chemical or Histological Analysis

Table 24. Length of clams in tank receiving $50 \%$ control sea water, $50 \%$ chlorinated sea water after 3 months of exposure. Date: $5 / 5 / 77$

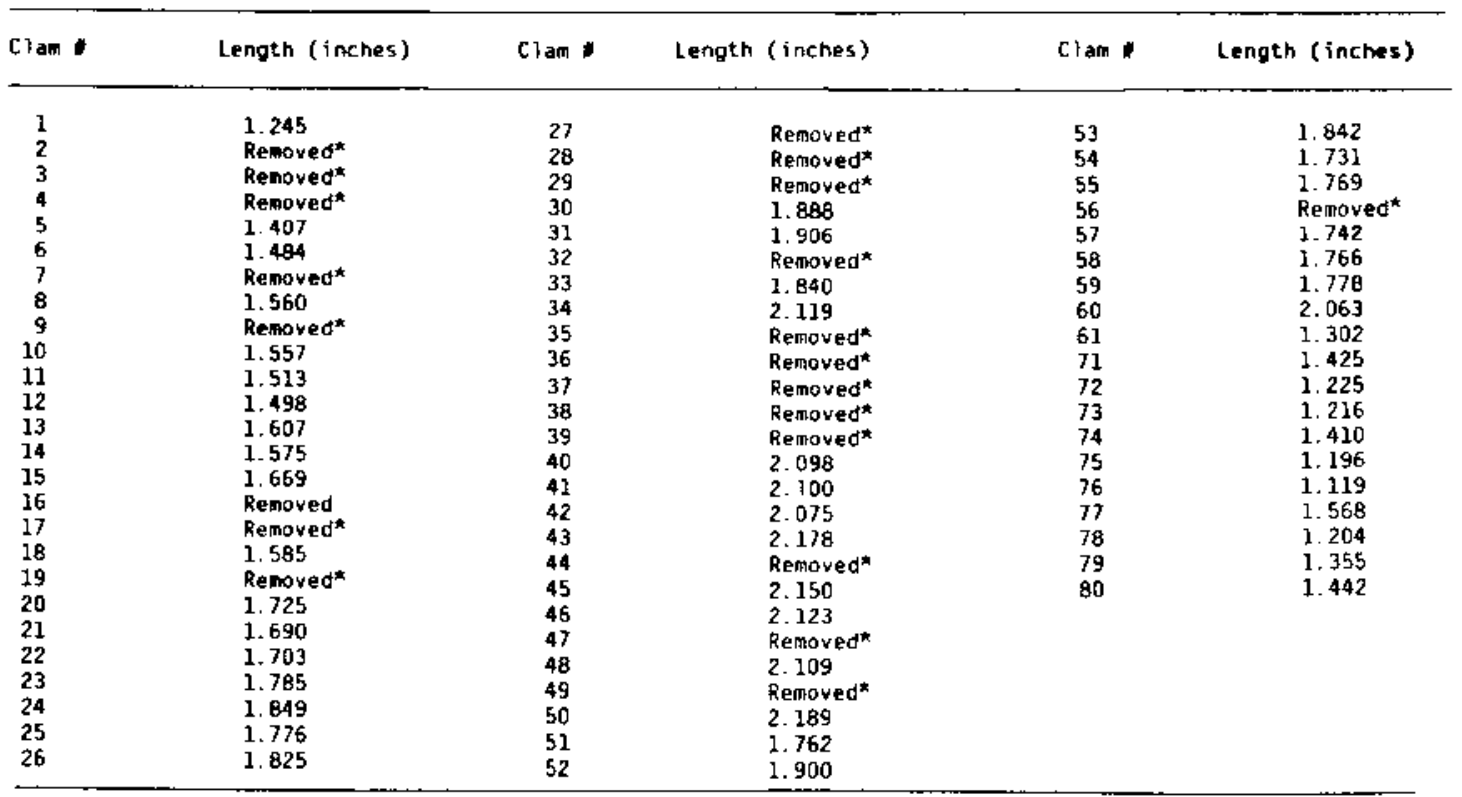

- Removed for Chemical or Histological Analysis. 
Table 25. Length of clams in tank receiving $100 \%$ chlorinated sea water after 3 months of exposure. Date: 5/5/77

\begin{tabular}{|c|c|c|c|c|c|}
\hline clan & Length (inches) & clas & Length (inches) & Clam & Length (fnches) \\
\hline $\begin{array}{r}1 \\
2 \\
3 \\
4 \\
5 \\
6 \\
7 \\
8 \\
9 \\
10 \\
11 \\
12 \\
13 \\
14 \\
15 \\
16 \\
17 \\
18 \\
19 \\
20 \\
21 \\
22 \\
23 \\
24\end{array}$ & $\begin{array}{l}\text { Removed* } \\
\text { Removed* } \\
1.313 \\
\text { Removed* } \\
1.354 \\
1.398 \\
1.569 \\
1.625 \\
1.595 \\
1.583 \\
\text { Removed* } \\
\text { Removed* } \\
1.623 \\
\text { Renoved } \\
\text { Removed } \\
1.635 \\
1.642 \\
1.669 \\
\text { Removed* } \\
1.654 \\
1.689 \\
\text { Removed } \\
\text { Removed* } \\
1.835\end{array}$ & $\begin{array}{l}25 \\
26 \\
27 \\
28 \\
29 \\
30 \\
31 \\
32 \\
33 \\
34 \\
35 \\
36 \\
37 \\
38 \\
39 \\
40 \\
41 \\
42 \\
43 \\
44 \\
45 \\
46 \\
47 \\
48\end{array}$ & $\begin{array}{l}1.787 \\
\text { Removed* } \\
\text { Removed* } \\
\text { Renoved* } \\
1.855 \\
1.961 \\
1.874 \\
\text { Removed } \\
1.934 \\
1.964 \\
1.961 \\
\text { Removed* } \\
\text { Removed } \\
2.098 \\
\text { Removed* } \\
2.117 \\
\text { Removed* } \\
\text { Removed* } \\
\text { Removed* } \\
2.039 \\
\text { Removed* } \\
2.169 \\
\text { Removed* } \\
2.109 \\
\text {.1 }\end{array}$ & $\begin{array}{l}49 \\
50 \\
51 \\
52 \\
53 \\
54 \\
55 \\
56 \\
57 \\
58 \\
59 \\
60 \\
61 \\
52 \\
71 \\
72 \\
73 \\
74 \\
75 \\
76 \\
77 \\
78 \\
79 \\
80\end{array}$ & $\begin{array}{l}2.044 \\
2.168 \\
1.662 \\
1.554 \\
1.762 \\
1.780 \\
1.659 \\
1.559 \\
1.604 \\
1.766 \\
1.677 \\
2.004 \\
1.526 \\
1.874 \\
1.464 \\
1.319 \\
1.343 \\
1.126 \\
1.362 \\
1.156 \\
1.400 \\
1.349 \\
1.254 \\
1.344\end{array}$ \\
\hline
\end{tabular}

* Removed for Chemical or Histological Analysis.

Table 26. Dissolved oxygen, temperature, salinity and $\mathrm{pH}$ of sea water in tank receiving $75 \%$ control sea water and 25\% chlorinated sea water.

\begin{tabular}{|c|c|c|c|c|}
\hline Date & Temperature & $\begin{array}{l}\text { Salinity } \\
\%\end{array}$ & $\begin{array}{c}\text { Dissolved oxygen } \\
\mathrm{mg} / \mathrm{x}\end{array}$ & pH \\
\hline $\begin{array}{l}2 / 2 / 77 \\
2 / 4 / 77 \\
2 / 6 / 77 \\
2 / 8 / 77 \\
2 / 9 / 77 \\
2 / 11 / 77 \\
2 / 13 / 77 \\
2 / 14 / 77 \\
2 / 16 / 77 \\
2 / 18 / 77 \\
2 / 21 / 77 \\
2 / 23 / 77 \\
2 / 25 / 77 \\
2 / 28 / 77 \\
3 / 2 / 77 \\
3 / 4 / 77 \\
3 / 7 / 77 \\
3 / 9 / 77 \\
3 / 11 / 77 \\
3 / 14 / 77 \\
3 / 16 / 77 \\
3 / 18 / 77 \\
3 / 21 / 77 \\
3 / 23 / 77 \\
3 / 25 / 77 \\
3 / 28 / 77 \\
3 / 30 / 77 \\
4 / 1 / 77 \\
4 / 4 / 77 \\
4 / 5 / 77 \\
4 / 8 / 77 \\
4 / 11 / 77 \\
4 / 13 / 77 \\
4 / 15 / 77 \\
4 / 18 / 77 \\
4 / 20 / 77 \\
4 / 22 / 77 \\
4 / 25 / 77 \\
4 / 27 / 77 \\
4 / 29 / 77 \\
5 / 2 / 77 \\
5 / 4 / 77 \\
5 / 6 / 77 \\
5 / 9 / 77 \\
\end{array}$ & $\begin{array}{l}15.0 \\
14.9 \\
15.0 \\
15.1 \\
15.0 \\
15.1 \\
15.1 \\
14.8 \\
15.2 \\
15.1 \\
15.2 \\
15.0 \\
15.0 \\
15.3 \\
15.3 \\
15.6 \\
15.2 \\
15.1 \\
15.3 \\
15.1 \\
15.0 \\
15.0 \\
15.0 \\
15.2 \\
14.8 \\
14.8 \\
14.5 \\
15.0 \\
15.0 \\
15.0 \\
15.1 \\
15.2 \\
15.2 \\
15.0 \\
14.8 \\
14.9 \\
14.7 \\
14.8 \\
14.8 \\
14.9 \\
14.8 \\
14.2 \\
15.0 \\
14.7 \\
\end{array}$ & $\begin{array}{l}31.0 \\
30.3 \\
31.0 \\
31.0 \\
30.3 \\
30.8 \\
30.0 \\
31.0 \\
30.6 \\
30.4 \\
30.4 \\
30.6 \\
30.7 \\
30.0 \\
29.8 \\
30.1 \\
30.0 \\
30.3 \\
30.4 \\
30.7 \\
30.6 \\
30.7 \\
30.0 \\
29.5 \\
30.0 \\
30.4 \\
30.4 \\
30.3 \\
29.9 \\
30.0 \\
30.0 \\
30.4 \\
30.4 \\
30.6 \\
30.2 \\
30.2 \\
30.3 \\
30.3 \\
30.2 \\
30.6 \\
30.2 \\
30.4 \\
30.0 \\
30.3\end{array}$ & $\begin{array}{l}8.15 \\
8.04 \\
8.10 \\
7.98 \\
7.74 \\
8.05 \\
8.12 \\
8.04 \\
7.45 \\
8.10 \\
8.07 \\
7.78 \\
7.88 \\
7.94 \\
8.00 \\
8.20 \\
7.98 \\
8.20 \\
8.30 \\
8.20 \\
8.15 \\
8.18 \\
8.14 \\
8.04 \\
8.28 \\
8.34 \\
8.35 \\
8.40 \\
8.10 \\
8.04 \\
8.14 \\
8.20 \\
8.25 \\
8.25 \\
9.04 \\
8.88 \\
8.16 \\
8.16 \\
8.30 \\
8.25 \\
8.23 \\
8.26 \\
8.10 \\
8.24 \\
\end{array}$ & $\begin{array}{l}7.8 \\
7.9 \\
7.9 \\
8.0 \\
7.8 \\
8.0 \\
8.0 \\
8.0 \\
7.9 \\
8.0 \\
8.0 \\
7.7 \\
7.9 \\
6.0 \\
8.0 \\
7.8 \\
8.0 \\
8.0 \\
7.9 \\
6.1 \\
7.9 \\
7.9 \\
8.0 \\
8.0 \\
7.9 \\
7.9 \\
7.9 \\
7.9 \\
8.0 \\
7.8 \\
8.0 \\
7.9 \\
8.0 \\
8.0 \\
8.0 \\
8.0 \\
8.0 \\
8.0 \\
8.0 \\
8.1 \\
8.1 \\
8.1 \\
8.1 \\
8.1 \\
.0\end{array}$ \\
\hline
\end{tabular}


Table 27. Chlorine produced oxidant concentrations in clam exposure tanks $(\mathrm{mg} / \ell)$.

\begin{tabular}{|c|c|c|c|c|c|c|}
\hline \multirow[b]{2}{*}{ Date } & \multirow[b]{2}{*}{$100 \pi / 0 \pi$} & \multirow{2}{*}{$\begin{array}{l}\text { Control } \\
94 x / 6 x\end{array}$} & \multicolumn{2}{|c|}{ iea Water/Chlorinated } & a water & \multirow[b]{2}{*}{$0 x / 10 \pi$} \\
\hline & & & $\operatorname{sex} / 12 x$ & $75 x / 25 x$ & $50 \pi / 50 \pi$ & \\
\hline $2 / 2 / 77$ & .00 & -. & $\ldots$ & -. & 01 & 01 \\
\hline $2 / 4 / 77$ & .00 & -- & -- & -- & .01 & .02 \\
\hline $2 / 8 / 77$ & .00 & -- & $\cdots$ & 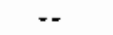 & 01 & 01 \\
\hline $2 / 9 / 77$ & .00 & -- & -. & $\cdots$ & 01 & .01 \\
\hline $2 / 11 / 77$ & .00 & -- & $=-$ & $\cdots$ & 01 & .01 \\
\hline $2 / 14 / 77$ & .00 & -- & -- & -- & .01 & .01 \\
\hline $2 / 16 / 77$ & .00 & -- & -. & -- & 01 & .02 \\
\hline $2 / 18 / 77$ & .00 & -- & $\cdots$ & -- & .01 & .02 \\
\hline $2 / 22 / 77$ & .00 & - & -- & -- & .01 & .01 \\
\hline $2 / 23 / 77$ & .00 & -- & -- & -- & 01 & .01 \\
\hline $2 / 25 / 77$ & .00 & -. & $-\infty$ & -. & .01 & .01 \\
\hline $2 / 28 / 77$ & .00 & -- & -- & - & .01 & .01 \\
\hline $3 / 2 / 77$ & .00 & -- & $\cdots$ & -- & .01 & .01 \\
\hline $3 / 4 / 77$ & .00 & -- & -- & -- & .01 & .01 \\
\hline $3 / 7 / 77$ & .00 & -. & - & - & .01 & .01 \\
\hline $3 / 9 / 77$ & .00 & -- & - & -- & .01 & 01 \\
\hline $3 / 11 / 77$ & .01 & -- & -- & -- & .01 & .01 \\
\hline $3 / 14 / 77$ & .00 & -- & -- & -- & .01 & .02 \\
\hline $3 / 16 / 77$ & .00 & $=$ & $\cdots$ & -- & .01 & -- \\
\hline $3 / 18 / 77$ & .00 & -- & -- & $\because$ & .01 & .02 \\
\hline $3 / 21 / 77$ & .00 & $\cdots$ & -- & $\therefore$ & .01 & .02 \\
\hline $3 / 23 / 77$ & .00 & $\ldots$ & -. &.- & .01 & .02 \\
\hline $3 / 25 / 77$ & .00 & -- & -- & $\cdots$ & .01 & .02 \\
\hline $3 / 28 / 77$ & .00 & -- & -. & -- & .01 & 02 \\
\hline $3 / 30 / 77$ & .00 & -- & -- & $\cdots$ & .01 & .02 \\
\hline $4 / 1 / 77$ & .00 & $\cdots$ & -- & $\cdots$ & .01 & 02 \\
\hline $4 / 4 / 77$ & .00 & -- & -- & -- & .01 & .01 \\
\hline $4 / 6 / 77$ & .00 & $\cdots$ & $-\infty$ & $-\infty$ & .00 & .01 \\
\hline $4 / 8 / 77$ & .00 & $\cdots$ & - & $\cdots$ & .01 & .02 \\
\hline $4 / 11 / 77$ & .00 & -- & -. & - & .01 & .02 \\
\hline $4 / 13 / 77$ & .00 & -- & -- & -- & .01 & .02 \\
\hline $4 / 18 / 77$ & .00 & $\cdots$ & $\cdots$ & $\cdots$ & .01 & .02 \\
\hline $4 / 20 / 77$ & .00 & -- & - & $\cdots$ & .01 & .02 \\
\hline $4 / 22 / 77$ & 01 & - & -- & -- & .02 & .02 \\
\hline $4 / 25 / 77$ & .01 & .01 & .01 & .01 & .02 & - \\
\hline $4 / 27 / 77$ & .00 & .01 & .01 & .01 & .01 & .02 \\
\hline $4 / 29 / 77$ & .00 & .01 & .01 & .01 & .02 & .03 \\
\hline $5 / 2 / 77$ & .01 & .01 & 01 & .01 & .01 & .02 \\
\hline $5 / 4 / 77$ & .01 & .01 & .01 & .01 & .01 & .03 \\
\hline $5 / 6 / 77$ & .01 & .01 & 01 & 01 & .01 & .03 \\
\hline $5 / 9 / 77$ & .00 & .01 & .01 & .01 & .01 & .03 \\
\hline
\end{tabular}

Table 28. Length change in clams from tank receiving $100 \%$ control sea water after 3 months of exposure.

\begin{tabular}{|c|c|c|c|}
\hline Clam & Dlength (inches) $\times 10^{-3}$ & Clat & Alength (1nches) $\times 10^{-3}$ \\
\hline 1 & -3 & & \\
\hline 2 & -3 & 26 & -6 \\
\hline 6 & -8 & 28 & -4 \\
\hline 9 & -9 & 29 & -5 \\
\hline II & -13 & 30 & -9 \\
\hline 13 & -5 & 32 & -7 \\
\hline 14 & $-\theta$ & 33 & -5 \\
\hline 15 & -5 & 38 & +1 \\
\hline 16 & -4 & 40 & -4 \\
\hline 17 & -10 & 41 & -4 \\
\hline 18 & -3 & 43 & -12 \\
\hline 19 & -5 & 44 & -6 \\
\hline 22 & -5 & 46 & -6 \\
\hline 24 & -4 & 49 & -2 \\
\hline 25 & -8 & 50 & -6 \\
\hline
\end{tabular}


Table 29. Length change in clams from tank receiving $94 \%$ control sea water, $6 \%$ chlorinated sea water after 3 months of exposure.

\begin{tabular}{cc}
\hline Clan & Siength (inches) $\times 10^{-3}$ \\
\hline 1 & -4 \\
2 & -5 \\
3 & -5 \\
6 & -9 \\
7 & -3 \\
8 & -3 \\
9 & -5 \\
10 & -4 \\
13 & -5 \\
15 & -7 \\
16 & -2 \\
17 & -5 \\
18 & 0 \\
22 & -4 \\
23 & -4 \\
26 & -6 \\
27 & -10 \\
30 & -2 \\
32 & -2 \\
33 & -0 \\
34 & -6 \\
35 & -5 \\
36 & -5 \\
37 & -6 \\
39 & -4 \\
40 & -6 \\
41 & -3 \\
44 & -3 \\
46 & -5 \\
50 & \\
\hline
\end{tabular}

Table 30 . Length change in clams from tank receiving $88 \%$ control sea water, $12 \%$ chlorinated sea water after 3 months of exposure.

\begin{tabular}{cc}
\hline Clan & Alength (incties) $\times 10^{-3}$ \\
\hline 1 & -5 \\
3 & -3 \\
4 & -6 \\
6 & -3 \\
8 & -6 \\
10 & -6 \\
12 & -6 \\
13 & -6 \\
14 & -4 \\
15 & -4 \\
17 & -5 \\
20 & -4 \\
21 & -6 \\
26 & -7 \\
27 & -5 \\
28 & -6 \\
30 & -5 \\
31 & -6 \\
34 & -4 \\
35 & -8 \\
36 & -5 \\
38 & -7 \\
39 & -8 \\
40 & -5 \\
41 & -1 \\
43 & -5 \\
44 & -4 \\
48 & \\
49 & \\
\hline
\end{tabular}


Table 31 . Length change in clams from tank receiving $75 \%$ control sea water, $25 \%$ chlorinated sea water after 3 months of exposure.

\begin{tabular}{cc}
\hline 1 & Slength (inches) $\times 10^{-3}$ \\
2 & -5 \\
3 & -5 \\
5 & -6 \\
9 & -5 \\
10 & -3 \\
12 & -4 \\
13 & -9 \\
14 & -5 \\
15 & -7 \\
15 & -4 \\
18 & -4 \\
22 & -2 \\
23 & -11 \\
24 & -1 \\
29 & -5 \\
30 & -3 \\
31 & -3 \\
33 & -4 \\
34 & -7 \\
35 & -4 \\
36 & -3 \\
41 & -1 \\
44 & -3 \\
45 & -2 \\
50 & -4 \\
\hline
\end{tabular}

Table 32. Length change in clams from tank receiving $50 \%$ control sea water, $50 \%$ chlorinated sea water after 3 months of exposure.

\begin{tabular}{cc}
\hline C1am & Alength (inches) $\times 10^{-3}$ \\
\hline 1 & -4 \\
5 & -5 \\
6 & -6 \\
8 & -5 \\
10 & -6 \\
11 & -5 \\
12 & -6 \\
13 & -3 \\
14 & -5 \\
15 & -6 \\
18 & -4 \\
20 & -2 \\
21 & -6 \\
22 & -5 \\
23 & -3 \\
24 & -5 \\
25 & -4 \\
26 & -6 \\
30 & -11 \\
31 & -4 \\
33 & -4 \\
34 & -6 \\
40 & -8 \\
41 & -5 \\
42 & -4 \\
43 & -3 \\
45 & -4 \\
46 & +4 \\
48 & 0 \\
50 & \\
\hline
\end{tabular}


Table 33. Length change in clams from tank receiving $100 \%$ chlorinated sea water after 3 months of exposure.

\begin{tabular}{ll}
\hline Cian & Alength (incines) $\times 10^{-}$, \\
\hline 3 & -12 \\
5 & -3 \\
6 & -4 \\
7 & -1 \\
9 & -4 \\
9 & -5 \\
10 & -2 \\
13 & -3 \\
16 & -3 \\
17 & -3 \\
18 & -2 \\
20 & -4 \\
21 & -4 \\
24 & -2 \\
25 & -2 \\
29 & -3 \\
30 & -3 \\
31 & -3 \\
33 & -6 \\
34 & -5 \\
35 & 0 \\
38 & -3 \\
40 & -4 \\
44 & -5 \\
46 & 0 \\
48 & -3 \\
49 & -5 \\
50 & \\
\hline
\end{tabular}

Table 34. Summary of length changes at all test conditions after 3 months of exposure.

\begin{tabular}{|c|c|c|c|}
\hline $\begin{array}{c}\text { TANK } \\
\text { Control/schlorinated }\end{array}$ & $\begin{array}{l}\text { Average Difference } \\
\left(\text { Inches } \times 10^{3}\right)\end{array}$ & $\left(x^{5.0} 10^{-3}\right)$ & Number \\
\hline $\begin{array}{l}100 x / 0 x \\
94 x / 6 x \\
88 x / 12 x \\
75 x / 25 x \\
50 x / 50 x \\
0 x / 100 x\end{array}$ & $\begin{array}{l}-6 \\
-5 \\
-5 \\
-5 \\
-4 \\
-4\end{array}$ & $\begin{array}{l}3 \\
3 \\
2 \\
2 \\
3 \\
2\end{array}$ & $\begin{array}{l}29 \\
30 \\
29 \\
27 \\
30 \\
28\end{array}$ \\
\hline
\end{tabular}


Table 35. Length, width, thickness, and weight measurements of clams in control exposure tank. Date: $2 / 27 / 78$

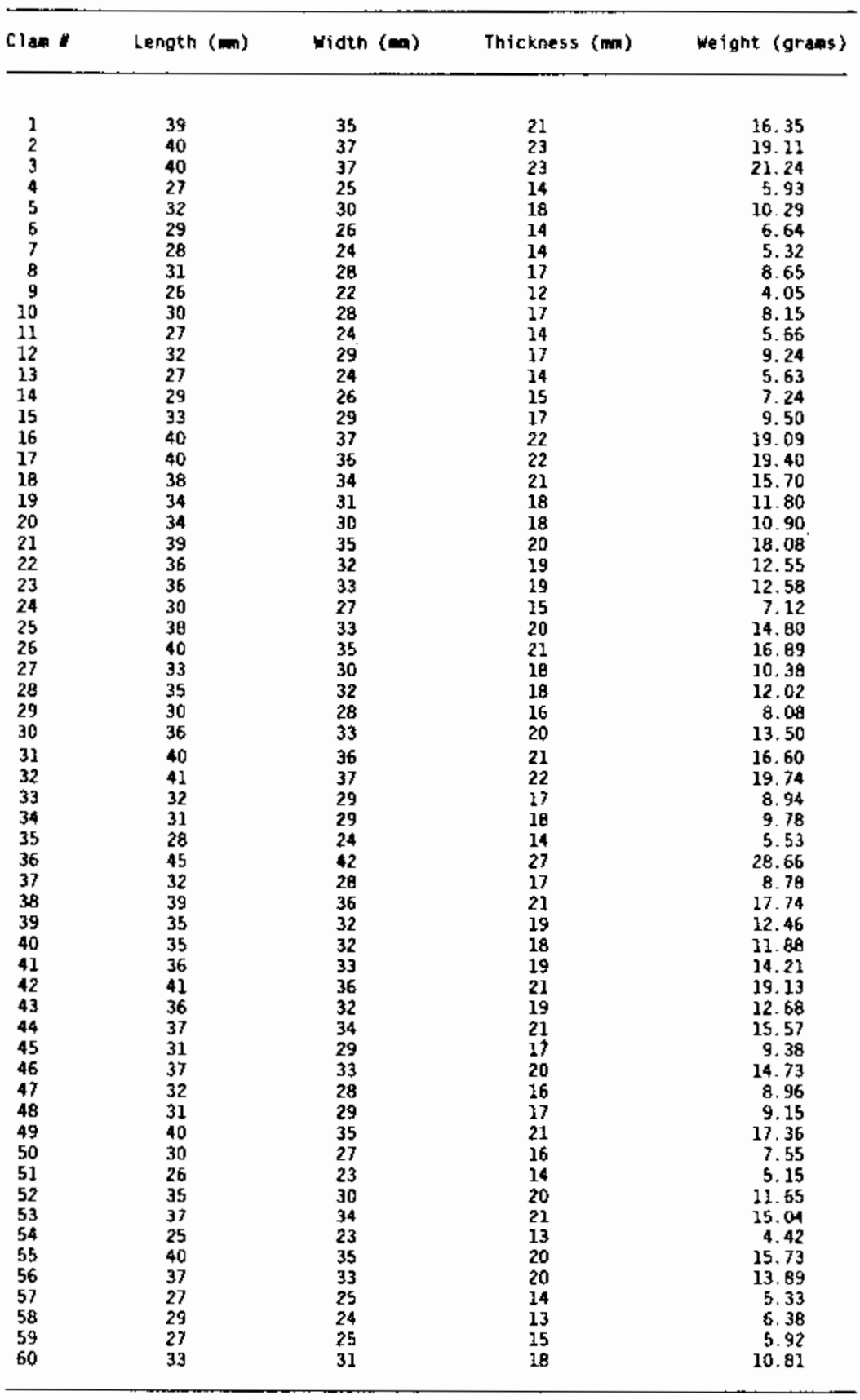

25 
Table 36. Length, width, thickness, and weight measurements of c]ams in $6 \mu \mathrm{g} / \ell$ CPO exposure tank. Date: $2 / 27 / 78$

\begin{tabular}{|c|c|c|c|c|}
\hline Clan & Length (m) & Width (m) & Thickness (m) & Weight (grans) \\
\hline $\begin{array}{l}101 \\
102 \\
103 \\
104 \\
105 \\
106 \\
107 \\
108 \\
109 \\
110 \\
111 \\
112 \\
113 \\
114 \\
115 \\
116 \\
117 \\
128 \\
119 \\
120 \\
121 \\
122 \\
123 \\
124 \\
125 \\
126 \\
127 \\
128 \\
129 \\
130\end{array}$ & $\begin{array}{l}30 \\
28 \\
25 \\
32 \\
38 \\
28 \\
42 \\
35 \\
25 \\
35 \\
29 \\
25 \\
26 \\
26 \\
34 \\
30 \\
37 \\
38 \\
37 \\
41 \\
36 \\
40 \\
26 \\
29 \\
34 \\
31 \\
27 \\
30 \\
25 \\
27\end{array}$ & $\begin{array}{l}29 \\
25 \\
22 \\
29 \\
35 \\
26 \\
37 \\
32 \\
23 \\
32 \\
25 \\
22 \\
23 \\
23 \\
32 \\
27 \\
34 \\
35 \\
34 \\
37 \\
32 \\
37 \\
23 \\
26 \\
30 \\
28 \\
24 \\
26 \\
22 \\
25\end{array}$ & $\begin{array}{l}17 \\
14 \\
13 \\
17 \\
21 \\
15 \\
24 \\
19 \\
13 \\
20 \\
14 \\
12 \\
13 \\
12 \\
17 \\
16 \\
20 \\
20 \\
21 \\
22 \\
18 \\
22 \\
13 \\
15 \\
18 \\
16 \\
15 \\
16 \\
12 \\
15\end{array}$ & $\begin{array}{r}9.25 \\
6.10 \\
4.22 \\
9.57 \\
16.40 \\
22.06 \\
6.18 \\
12.51 \\
4.74 \\
13.42 \\
6.03 \\
4.17 \\
4.75 \\
4.49 \\
9.66 \\
8.07 \\
13.89 \\
15.65 \\
16.13 \\
19.77 \\
12.56 \\
19.26 \\
4.67 \\
6.23 \\
10.98 \\
8.23 \\
5.43 \\
7.41 \\
4.13 \\
5.91\end{array}$ \\
\hline $\begin{array}{l}131 \\
132 \\
133 \\
134 \\
135 \\
136 \\
137 \\
138 \\
139 \\
140 \\
141 \\
142 \\
143 \\
144 \\
145 \\
146 \\
147 \\
148 \\
149 \\
150 \\
151 \\
152 \\
153 \\
154 \\
155 \\
156 \\
157 \\
158 \\
159 \\
160\end{array}$ & $\begin{array}{l}28 \\
29 \\
29 \\
27 \\
34 \\
34 \\
32 \\
32 \\
35 \\
33 \\
30 \\
29 \\
36 \\
25 \\
38 \\
31 \\
32 \\
36 \\
35 \\
32 \\
34 \\
35 \\
35 \\
33 \\
39 \\
37 \\
39 \\
41 \\
38 \\
35\end{array}$ & $\begin{array}{l}25 \\
27 \\
25 \\
25 \\
32 \\
30 \\
29 \\
29 \\
33 \\
31 \\
26 \\
27 \\
33 \\
23 \\
35 \\
29 \\
29 \\
33 \\
31 \\
29 \\
30 \\
32 \\
32 \\
30 \\
36 \\
34 \\
36 \\
38 \\
33 \\
31\end{array}$ & $\begin{array}{l}14 \\
15 \\
15 \\
15 \\
19 \\
19 \\
17 \\
17 \\
20 \\
18 \\
16 \\
16 \\
21 \\
12 \\
21 \\
18 \\
18 \\
21 \\
17 \\
17 \\
17 \\
19 \\
20 \\
17 \\
20 \\
19 \\
21 \\
23 \\
20 \\
19\end{array}$ & $\begin{array}{r}5.89 \\
7.63 \\
5.63 \\
5.96 \\
12.22 \\
11.40 \\
9.35 \\
9.11 \\
13.76 \\
10.84 \\
7.96 \\
7.72 \\
14.85 \\
4.28 \\
16.43 \\
9.91 \\
9.91 \\
14.82 \\
11.30 \\
9.61 \\
10.35 \\
11.95 \\
13.49 \\
10.07 \\
17.22 \\
14.48 \\
16.76 \\
20.90 \\
14.72 \\
12.32\end{array}$ \\
\hline
\end{tabular}


Table 37. Length, width, thickness, and weight measurements of clams in $12 \mu \mathrm{g} / \mathrm{Q}$ CPO exposure tank. Date: $2 / 27 / 78$




Table 38. Length, width, thickness, and weight measurements of clams in $25 \mu \mathrm{g} / 2$ CPO exposure tank. Date: $2 / 27 / 78$

\begin{tabular}{|c|c|c|c|c|}
\hline clan. & Length $(\infty)$ & Width (ma) & Thickness (m) & Weight (grans) \\
\hline 301 & 29 & 26 & 15 & 6.8 \\
\hline 302 & 38 & 34 & 21 & 16.6 \\
\hline $\begin{array}{l}303 \\
304\end{array}$ & $\begin{array}{l}37 \\
32\end{array}$ & 33 & 19 & 13.4 \\
\hline 305 & $\begin{array}{l}32 \\
36\end{array}$ & 31 & 17 & 9.6 \\
\hline 306 & 38 & 34 & 20 & \\
\hline 307 & 34 & 32 & \pm 9 & 12.5 \\
\hline 308 & 42 & 38 & 22 & 21.1 \\
\hline 309 & 44 & 41 & 24 & 25.4 \\
\hline $\begin{array}{l}310 \\
311\end{array}$ & $\begin{array}{l}42 \\
25\end{array}$ & $\begin{array}{l}49 \\
22\end{array}$ & 23 & 21.8 \\
\hline 312 & $\begin{array}{l}25 \\
28\end{array}$ & $\begin{array}{l}22 \\
26\end{array}$ & $\begin{array}{l}12 \\
15\end{array}$ & \\
\hline 313 & 30 & 28 & 27 & $\begin{array}{l}6.8 \\
8.6\end{array}$ \\
\hline $\begin{array}{l}314 \\
315\end{array}$ & 34 & 30 & 17 & 10.0 \\
\hline $\begin{array}{l}315 \\
316\end{array}$ & 35 & 31 & 18 & 12.1 \\
\hline $\begin{array}{l}\begin{array}{l}316 \\
317\end{array} \\
310\end{array}$ & $\begin{array}{l}35 \\
38\end{array}$ & 31 & 18 & 11.1 \\
\hline 318 & $\begin{array}{l}38 \\
41\end{array}$ & $\begin{array}{l}33 \\
37\end{array}$ & $\begin{array}{l}20 \\
22\end{array}$ & $\begin{array}{ll}14.3 \\
19.3\end{array}$ \\
\hline 319 & 38 & 34 & 21 & $\begin{array}{l}19.3 \\
17.0\end{array}$ \\
\hline 320 & 37 & 35 & 21 & 17.0 \\
\hline 321 & 26 & 26 & 25 & 5.5 \\
\hline $\begin{array}{l}322 \\
323\end{array}$ & 29 & 26 & 15 & 7.0 \\
\hline 324 & $\begin{array}{l}37 \\
34\end{array}$ & 34 & 20 & $\begin{array}{l}15.0 \\
10.7\end{array}$ \\
\hline 325 & 32 & 29 & 17 & $\begin{array}{r}10.7 \\
9.4\end{array}$ \\
\hline 326 & 32 & 30 & 18 & $\begin{array}{r}9.4 \\
10.0\end{array}$ \\
\hline 327 & 36 & 33 & 21 & \\
\hline 328 & 41 & 38 & & $\begin{array}{l}19.0 \\
19.1\end{array}$ \\
\hline 329 & & & 23 & $\begin{array}{l}19.1 \\
21.6\end{array}$ \\
\hline 330 & 37 & 33 & 20 & 15.5 \\
\hline 331 & 42 & 38 & 23 & 22.8 \\
\hline 332 & 36 & 32 & 19 & 12.8 \\
\hline 333 & 37 & 33 & 20 & 14.2 \\
\hline 334 & 36 & 31 & 18 & 11.7 \\
\hline 335 & 34 & 30 & 18 & 10.5 \\
\hline $\begin{array}{l}336 \\
337\end{array}$ & 42 & 38 & 23 & 21.1 \\
\hline 337 & 38 & 33 & 20 & 14.9 \\
\hline $\begin{array}{l}338 \\
339\end{array}$ & 38 & 34 & 21 & 15. 7 \\
\hline $\begin{array}{l}339 \\
340\end{array}$ & $\begin{array}{l}40 \\
40\end{array}$ & $\begin{array}{l}36 \\
36\end{array}$ & $\begin{array}{l}22 \\
22\end{array}$ & 18.1 \\
\hline 341 & 27 & $\begin{array}{l}36 \\
24\end{array}$ & 14 & 6.0 \\
\hline 342 & 35 & 32 & 19 & 13.0 \\
\hline 343 & 38 & 35 & 20 & 16.1 \\
\hline $\begin{array}{l}\begin{array}{l}344 \\
345\end{array} \\
345\end{array}$ & 31 & 27 & 17 & 8.9 \\
\hline $\begin{array}{l}\begin{array}{r}345 \\
346\end{array} \\
345\end{array}$ & $\begin{array}{l}32 \\
36\end{array}$ & $\begin{array}{l}29 \\
33\end{array}$ & $\begin{array}{l}18 \\
19 \\
19\end{array}$ & $\begin{array}{r}9.8 \\
13.8\end{array}$ \\
\hline 347 & 41 & 38 & 22 & 19.3 \\
\hline 348 & 35 & 31 & 17 & 10.8 \\
\hline 349 & 36 & 32 & 19 & 12.2 2 \\
\hline $\begin{array}{l}\begin{array}{l}350 \\
351\end{array} \\
351\end{array}$ & $\begin{array}{l}41 \\
30\end{array}$ & $\begin{array}{l}37 \\
27\end{array}$ & 22 & 19.3 \\
\hline 352 & $\begin{array}{l}30 \\
32\end{array}$ & $\begin{array}{l}26 \\
28\end{array}$ & $\begin{array}{l}15 \\
16\end{array}$ & 8.6 \\
\hline 353 & 34 & 31 & 18 & 10.4 \\
\hline $\begin{array}{l}354 \\
354\end{array}$ & 38 & 34 & 21 & 15.8 \\
\hline 355 & 40 & 35 & 22 & 18. 2 \\
\hline $\begin{array}{l}356 \\
357\end{array}$ & $\begin{array}{l}42 \\
42\end{array}$ & 39 & $\begin{array}{l}23 \\
23\end{array}$ & $\begin{array}{l}21.9 \\
21.9\end{array}$ \\
\hline 358 & $\begin{array}{l}42 \\
43\end{array}$ & 39 & $\begin{array}{l}23 \\
24\end{array}$ & $\begin{array}{l}21.9 \\
23.2\end{array}$ \\
\hline 359 & 35 & 33 & 19 & 13.3 \\
\hline 360 & 35 & 31 & 19 & 11.2 \\
\hline
\end{tabular}


Table 39. Length, width, thickness, and weight measurements of clams in $50 \mu \mathrm{g} / \ell$ CPO exposure tank. Date: $2 / 27 / 78$

\begin{tabular}{|c|c|c|c|c|}
\hline Clam * & Lengeth (mm) & width $(\mathrm{mm})$ & Thickness $(m)$ & Weight (grams) \\
\hline 401 & 26 & 23 & 13 & 5.0 \\
\hline 402 & 33 & 30 & 18 & 10.4 \\
\hline 403 & 30 & 26 & 16 & 8.0 \\
\hline $\begin{array}{l}404 \\
405\end{array}$ & 39 & 37 & 23 & 18.5 \\
\hline $\begin{array}{l}405 \\
406\end{array}$ & $\begin{array}{l}33 \\
37\end{array}$ & 30 & 18 & 10.3 \\
\hline $\begin{array}{l}406 \\
407\end{array}$ & $\begin{array}{l}37 \\
34\end{array}$ & $\begin{array}{l}33 \\
32\end{array}$ & $\begin{array}{l}20 \\
18\end{array}$ & 14.5 \\
\hline 408 & 31 & $\begin{array}{l}32 \\
28\end{array}$ & $\begin{array}{l}18 \\
17\end{array}$ & $\begin{array}{r}11.6 \\
8.6\end{array}$ \\
\hline 409 & 33 & 31 & 17 & $\begin{array}{r}8.6 \\
10.8\end{array}$ \\
\hline 410 & 41 & 37 & 23 & 19.9 \\
\hline $\begin{array}{l}411 \\
412\end{array}$ & $\begin{array}{l}29 \\
41\end{array}$ & 25 & 14 & 6.0 \\
\hline $\begin{array}{l}412 \\
413\end{array}$ & 41 & $\begin{array}{l}36 \\
29\end{array}$ & 23 & 18.7 \\
\hline $\begin{array}{l}413 \\
414\end{array}$ & $\begin{array}{l}33 \\
40\end{array}$ & $\begin{array}{l}29 \\
36\end{array}$ & $\begin{array}{l}18 \\
22\end{array}$ & $\begin{array}{l}11.0 \\
18.2\end{array}$ \\
\hline 415 & 33 & 30 & 18 & $\begin{array}{l}18.2 \\
10.9\end{array}$ \\
\hline 416 & 37 & 34 & 19 & \\
\hline 417 & 39 & 35 & 22 & $\begin{array}{l}12.6 \\
18.0\end{array}$ \\
\hline $\begin{array}{l}418 \\
419\end{array}$ & & & & \\
\hline & 29 & 26 & & 6.9 \\
\hline 420 & 32 & 29 & 17 & 9.5 \\
\hline 421 & 41 & 36 & 21 & 18.6 \\
\hline 422 & 40 & 36 & 22 & 18.9 \\
\hline 423 & 42 & 38 & 22 & 20.3 \\
\hline $\begin{array}{l}424 \\
425\end{array}$ & 36 & 33 & 20 & 14.2 \\
\hline $\begin{array}{l}425 \\
426\end{array}$ & $\begin{array}{l}40 \\
29\end{array}$ & 36 & 22 & 19.2 \\
\hline $\begin{array}{l}426 \\
427\end{array}$ & $\begin{array}{l}29 \\
41\end{array}$ & $\begin{array}{l}27 \\
36\end{array}$ & $\begin{array}{l}16 \\
27\end{array}$ & $\begin{array}{r}7.7 \\
8.9\end{array}$ \\
\hline 428 & $\begin{array}{l}41 \\
35\end{array}$ & $\begin{array}{l}36 \\
32\end{array}$ & $\begin{array}{l}22 \\
19\end{array}$ & 18.9 \\
\hline 429 & 39 & 36 & 20 & 17.5 \\
\hline 430 & 37 & 33 & 20 & 14.4 \\
\hline 431 & 35 & 32 & 20 & 13.1 \\
\hline $\begin{array}{l}432 \\
4333\end{array}$ & 41 & 37 & 22 & 19.2 \\
\hline 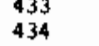 & $\begin{array}{l}39 \\
37\end{array}$ & $\begin{array}{l}35 \\
33\end{array}$ & 21 & 16.9 \\
\hline $\begin{array}{r}\begin{array}{r}344 \\
435\end{array} \\
4\end{array}$ & $\begin{array}{l}37 \\
41\end{array}$ & $\begin{array}{l}33 \\
39\end{array}$ & $\begin{array}{l}20 \\
25\end{array}$ & $\begin{array}{l}14.6 \\
26.1\end{array}$ \\
\hline 436 & $\begin{array}{l}41 \\
41\end{array}$ & 38 & $\begin{array}{l}25 \\
22\end{array}$ & $\begin{array}{r}26.1 \\
18.6\end{array}$ \\
\hline 437 & 28 & 26 & 15 & $\begin{array}{l}18.0 \\
6.0\end{array}$ \\
\hline $\begin{array}{l}438 \\
439\end{array}$ & 43 & 39 & 23 & 21.5 \\
\hline $\begin{array}{l}\begin{array}{r}439 \\
440\end{array} \\
4\end{array}$ & $\begin{array}{l}40 \\
48 \\
38\end{array}$ & 37 & 22 & 18.9 \\
\hline 441 & $\begin{array}{l}38 \\
27 \\
27\end{array}$ & $\begin{array}{l}35 \\
25\end{array}$ & 22 & $\begin{array}{ll}16.0 \\
5.6\end{array}$ \\
\hline 442 & 27 & $\begin{array}{l}25 \\
23\end{array}$ & $\begin{array}{l}13 \\
14\end{array}$ & $\begin{array}{l}5.6 \\
5.1\end{array}$ \\
\hline 443 & 34 & 30 & $\begin{array}{l}14 \\
18\end{array}$ & $\begin{array}{r}5.2 \\
10.9\end{array}$ \\
\hline $\begin{array}{l}444 \\
445\end{array}$ & 37 & 32 & 19 & 13.3 \\
\hline 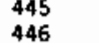 & 37 & 35 & 21 & 17.1 \\
\hline $\begin{array}{l}446 \\
447\end{array}$ & $\begin{array}{l}1 \\
41\end{array}$ & ${ }_{38}^{27}$ & 16 & $\begin{array}{r}8.5 \\
\end{array}$ \\
\hline 448 & $\begin{array}{l}41 \\
34\end{array}$ & $\begin{array}{l}38 \\
31\end{array}$ & $\begin{array}{l}23 \\
17\end{array}$ & 21.2 \\
\hline 449 & $\begin{array}{l}34 \\
40\end{array}$ & $\begin{array}{l}31 \\
37\end{array}$ & 22 & $\begin{array}{l}10.6 \\
19.4\end{array}$ \\
\hline 450 & 36 & 32 & 20 & 14.3 \\
\hline $\begin{array}{l}451 \\
452\end{array}$ & 32 & 28 & 17 & 9.0 \\
\hline $\begin{array}{l}452 \\
453\end{array}$ & $\begin{array}{l}35 \\
41\end{array}$ & 33 & 20 & 14.4 \\
\hline 454 & $\begin{array}{l}41 \\
41\end{array}$ & $\begin{array}{l}58 \\
37\end{array}$ & $\begin{array}{l}22 \\
22\end{array}$ & $\begin{array}{l}20.8 \\
19.3\end{array}$ \\
\hline 455 & $\begin{array}{l}41 \\
42\end{array}$ & $\begin{array}{l}37 \\
39\end{array}$ & 25 & $\begin{array}{l}9.3 \\
24.7\end{array}$ \\
\hline 456 & 40 & 37 & 22 & 19.6 \\
\hline & 34 & 30 & 18 & $\begin{array}{l}19.0 \\
12.0\end{array}$ \\
\hline 458 & 37 & 33 & $\begin{array}{l}18 \\
20\end{array}$ & $\begin{array}{l}12.0 \\
14.2\end{array}$ \\
\hline 459 & 38 & 34 & 20 & 15.4 \\
\hline 460 & 40 & 35 & 22 & 17.7 \\
\hline
\end{tabular}


Table 40. Length, width, thickness, and weight measurements of clams in $100 \mu \mathrm{g} / \ell$ CPO exposure tank. Date: 2/27/78

\begin{tabular}{|c|c|c|c|c|}
\hline clan & Length (m) & Width (m) & Thickness (mo) & Weight (grans) \\
\hline 501 & 44 & 41 & 25 & 24.5 \\
\hline 502 & 39 & 37 & 22 & 18.6 \\
\hline 503 & 35 & 32 & 19 & 12.8 \\
\hline 504 & 33 & 30 & 18 & 10.7 \\
\hline 505 & 38 & 35 & 20 & 15.7 \\
\hline 506 & 37 & 34 & 19 & 24.0 \\
\hline 507 & 38 & 35 & 27 & 15.0 \\
\hline 508 & 36 & 33 & 20 & 13.6 \\
\hline 509 & 37 & 34 & 20 & 14.2 \\
\hline 510 & 34 & 31 & 19 & 12.0 \\
\hline 511 & 30 & 26 & 15 & 6.4 \\
\hline 512 & 33 & 30 & 17 & 9.3 \\
\hline 513 & 32 & 29 & 17 & 10.0 \\
\hline 514 & 34 & 31 & 18 & 11.1 \\
\hline$\$ 15$ & 34 & 31 & 17 & 30.6 \\
\hline 516 & 30 & 26 & 16 & 7.1 \\
\hline 517 & 31 & 28 & 17 & 9.7 \\
\hline 518 & 31 & 28 & 17 & 8. 7 \\
\hline 519 & 35 & 32 & 19 & 12.6 \\
\hline 520 & 37 & 33 & 21 & 15.0 \\
\hline 521 & 32 & 30 & 18 & 11.1 \\
\hline 522 & 40 & 37 & 23 & 20.1 \\
\hline 523 & 32 & 30 & 17 & 10.2 \\
\hline 524 & 42 & 37 & 21 & 19.7 \\
\hline 525 & 42 & 40 & 22 & 21.8 \\
\hline 526 & 35 & 32 & 19 & 13.0 \\
\hline 527 & 38 & 34 & 19 & 14.4 \\
\hline 528 & 44 & 39 & 25 & 24.9 \\
\hline 529 & 45 & 41 & 24 & 16.5 \\
\hline 530 & 36 & 34 & 21 & 16.4 \\
\hline 531 & 34 & 31 & $1 B$ & 11.4 \\
\hline 532 & 41 & 37 & 22 & 19.6 \\
\hline 533 & 41 & 37 & 22 & 19.2 \\
\hline 534 & 38 & 35 & 21 & 17. 3 \\
\hline 535 & 37 & 33 & 20 & 14.7 \\
\hline 536 & 34 & 32 & 20 & 12.2 \\
\hline 537 & 38 & 34 & 20 & 14.5 \\
\hline 538 & 40 & 36 & 22 & 18.9 \\
\hline 539 & 33 & 30 & 17 & 10.3 \\
\hline 540 & 39 & 36 & 22 & 18.3 \\
\hline 541 & 40 & 36 & 22 & 17.7 \\
\hline 542 & 40 & 36 & 22 & 19.6 \\
\hline 543 & 32 & 28 & 18 & 9.4 \\
\hline 544 & 37 & 33 & 20 & 14.4 \\
\hline 545 & 37 & 32 & 20 & 13.3 \\
\hline 546 & 42 & 38 & 23 & 20.3 \\
\hline 547 & 34 & 31 & 18 & 11.8 \\
\hline 548 & 36 & 35 & 20 & 15.0 \\
\hline 549 & 35 & 33 & 21 & 13.9 \\
\hline 550 & 44 & 40 & 24 & 24.3 \\
\hline 551 & 34 & 31 & 18 & 11.3 \\
\hline 552 & 39 & 36 & 22 & 19.0 \\
\hline 553 & 37 & 35 & 21 & 15.5 \\
\hline 554 & 4] & 37 & 22 & 19.3 \\
\hline 555 & 33 & 29 & 18 & 10.8 \\
\hline 556 & 31 & 27 & 25 & 8. 6 \\
\hline 557 & 33 & 30 & 17 & 9.6 \\
\hline 558 & 39 & 36 & 21 & 16.0 \\
\hline 559 & 36 & 32 & 19 & 13.3 \\
\hline 550 & 38 & 35 & 21 & 15.8 \\
\hline
\end{tabular}


Table 4l. Length, width, thickness, and weight measurements of clams after one month of exposure. Date: $4 / 3 / 78$

\begin{tabular}{|c|c|c|c|c|}
\hline $\mathrm{Clan}$ & Length (m) & Width $(m)$ & Thickness (m) & Weight (grams \\
\hline \multicolumn{5}{|l|}{ CONTROL } \\
\hline a & 31 & 29 & 17 & 9.1 \\
\hline 46 & 36 & 33 & 20 & 14.8 \\
\hline 18 & 37 & 34 & 21 & 15.7 \\
\hline 3 & 40 & 37 & 24 & 21.4 \\
\hline 41 & 35 & 33 & 20 & 14.4 \\
\hline 30 & 36 & 33 & 20 & 13.8 \\
\hline 22 & 36 & 32 & 19 & 12.7 \\
\hline 39 & 35 & 32 & 19 & 12.8 \\
\hline \multicolumn{5}{|c|}{$6 \mathrm{yg} / \ell \quad \mathrm{CPO}$} \\
\hline$\frac{.12}{208}$ & 35 & 32 & 19 & 12.5 \\
\hline 147 & 32 & 30 & 28 & 10.0 \\
\hline 156 & 37 & 34 & 20 & 14.5 \\
\hline 158 & 41 & 38 & 23 & 20.9 \\
\hline 114 & 26 & 23 & 12 & 4.7 \\
\hline 116 & 30 & 26 & 17 & 8.2 \\
\hline 119 & 37 & 34 & 21 & 16.4 \\
\hline 122 & 41 & 37 & 23 & 19.3 \\
\hline \multicolumn{5}{|c|}{$12 \mathrm{ug} / \mathrm{lCPO}$} \\
\hline 208 & 34 & 30 & 18 & 11.1 \\
\hline 226 & 39 & 34 & 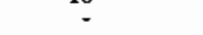 & 29.8 \\
\hline 235 & 41 & 38 & 22 & 20.2 \\
\hline 250 & 40 & 36 & 21 & 18.0 \\
\hline 224 & 41 & 38 & 24 & 22.0 \\
\hline 232 & 38 & 34 & 22 & 16.8 \\
\hline 247 & 43 & 39 & 24 & 23.9 \\
\hline 249 & 42 & 38 & 23 & 22.1 \\
\hline \multicolumn{5}{|c|}{$25 \mathrm{\mu g} / 2 \mathrm{CPO}$} \\
\hline 346 & 36 & 33 & 19 & 13.8 \\
\hline 355 & 40 & 36 & - & 22.4 \\
\hline 332 & 36 & 32 & 19 & 12.7 \\
\hline 311 & 24 & 22 & 12 & 4.1 \\
\hline 306 & 38 & 34 & 20 & 15.7 \\
\hline 317 & 37 & 33 & 20 & 14.4 \\
\hline 334 & 36 & 33 & IB & 11.6 \\
\hline 340 & 40 & 36 & 22 & 19.6 \\
\hline \multicolumn{5}{|c|}{ SO $\mathrm{eg} / 2$ CPO } \\
\hline 422 & 40 & 36 & 22 & 18.9 \\
\hline 425 & 39 & 36 & 22 & 19.0 \\
\hline 494 & 36 & 32 & - & 15.2 \\
\hline 453 & 41 & 38 & 21 & 20.8 \\
\hline 418 & 42 & 38 & 23 & 20.7 \\
\hline 421 & 41 & 37 & 21 & 18.6 \\
\hline 434 & 37 & 33 & 20 & 14.6 \\
\hline 460 & 39 & 35 & 22 & 17.6 \\
\hline \multicolumn{5}{|c|}{$100 \mu q / \ell$ CPO } \\
\hline & 45 & 41 & 25 & 26.0 \\
\hline 539 & 33 & 30 & 17 & 10.3 \\
\hline 547 & 35 & 31 & 10 & 11.7 \\
\hline 505 & 39 & 35 & 20 & 15.7 \\
\hline 522 & 40 & 37 & 23 & 20.0 \\
\hline 526 & 36 & 32 & 19 & 13.2 \\
\hline 542 & 40 & 36 & 22 & 19.6 \\
\hline
\end{tabular}


Table 42. Length, width, thickness, and weight measurements of clams after two months of exposure. Date: 5/1/78

\begin{tabular}{|c|c|c|c|c|}
\hline Clan 1 & Length $(m)$ & Vidth $(m)$ & Thickness $(m)$ & Weight (grans) \\
\hline \multicolumn{5}{|c|}{ CONTROL } \\
\hline 17 & 40 & 36 & 22 & 195 \\
\hline $\begin{array}{l}21 \\
27\end{array}$ & $\begin{array}{l}38 \\
32\end{array}$ & $\begin{array}{l}35 \\
30\end{array}$ & $\begin{array}{l}20 \\
18\end{array}$ & $\begin{array}{l}15.5 \\
10.4\end{array}$ \\
\hline 42 & 41 & 37 & 22 & 19.1 \\
\hline 26 & 40 & 35 & 21 & 17.1 \\
\hline 1 & 38 & 36 & 21 & 16.3 \\
\hline $\begin{array}{l}28 \\
55\end{array}$ & 35 & 32 & 19 & 12.5 \\
\hline & & & 19 & 25.8 \\
\hline \multicolumn{5}{|c|}{$6 \mu g / z \subset P O$} \\
\hline${ }_{155}^{106}$ & 42 & 39 & 24 & 22.0 \\
\hline 118 & $\begin{array}{l}39 \\
38\end{array}$ & 36 & 21 & 17.1 \\
\hline 109 & 26 & $\begin{array}{l}35 \\
23\end{array}$ & 21 & 15.6 \\
\hline 159 & 36 & 34 & 20 & $\begin{array}{l}4.9 \\
15.0\end{array}$ \\
\hline 110 & 35 & 32 & 20 & 13.6 \\
\hline 143 & 37 & 33 & 21 & 14.8 \\
\hline 138 & 32 & 29 & 17 & 9.1 \\
\hline \multicolumn{5}{|c|}{$\frac{12 \mathrm{\mu g} / 2 \mathrm{CPO}}{216}$} \\
\hline 234 & $\begin{array}{l}29 \\
39\end{array}$ & $\begin{array}{l}27 \\
36\end{array}$ & $\begin{array}{l}16 \\
21\end{array}$ & $\begin{array}{r}7.6 \\
17.1\end{array}$ \\
\hline 248 & 39 & 35 & 20 & 16.3 \\
\hline 257 & 38 & 35 & 22 & 16.4 \\
\hline 240 & 31 & 28 & 16 & 8.7 \\
\hline $\begin{array}{l}211 \\
218\end{array}$ & $\begin{array}{l}26 \\
32\end{array}$ & 24 & 14 & 5.3 \\
\hline 217 & $\begin{array}{l}32 \\
26\end{array}$ & $\begin{array}{l}30 \\
23 \\
23\end{array}$ & $\begin{array}{l}17 \\
13\end{array}$ & 9.8 \\
\hline \multirow{2}{*}{\multicolumn{5}{|c|}{$\frac{25, \mathrm{gg} / \ell \quad \mathrm{CPO}}{231}$}} \\
\hline & & 39 & & \\
\hline 333 & 37 & 33 & 19 & 14.2 \\
\hline 344 & 30 & 27 & 17 & 6. 9 \\
\hline 348 & 35 & 31 & 18 & 10.7 \\
\hline $\begin{array}{l}309 \\
305\end{array}$ & $\begin{array}{l}44 \\
35\end{array}$ & ${ }_{31}^{41}$ & 24 & 25.5 \\
\hline 305 & 35 & 31 & 19 & 12.5 \\
\hline 304 & $\begin{array}{l}32 \\
37\end{array}$ & $\begin{array}{l}29 \\
34\end{array}$ & 17 & 9.7 \\
\hline 323 & 37 & 34 & 22 & 24.9 \\
\hline \multicolumn{5}{|c|}{$50 \mathrm{\mu g} / \mathrm{CPO}$} \\
\hline $\begin{array}{l}455 \\
454\end{array}$ & $\begin{array}{l}42 \\
41\end{array}$ & $\begin{array}{l}39 \\
37\end{array}$ & $\begin{array}{l}25 \\
22\end{array}$ & $\begin{array}{l}24.3 \\
19.1\end{array}$ \\
\hline 428 & 36 & 32 & 19 & 12.6 \\
\hline 445 & 38 & 36 & 21 & 16.9 \\
\hline 438 & 43 & 38 & 23 & 21.7 \\
\hline 452 & 36 & 34 & 20 & 14.4 \\
\hline $\begin{array}{l}459 \\
427\end{array}$ & $\begin{array}{l}38 \\
41\end{array}$ & $\begin{array}{l}34 \\
36\end{array}$ & 22 & $\begin{array}{l}13.4 \\
19.0\end{array}$ \\
\hline \multicolumn{5}{|c|}{ 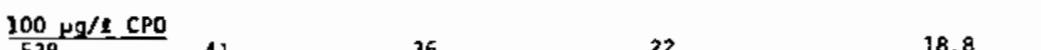 } \\
\hline 538 & $\begin{array}{l}41 \\
34\end{array}$ & 36 & 22 & $\begin{array}{l}18.8 \\
30.7\end{array}$ \\
\hline 553 & $\begin{array}{l}34 \\
37\end{array}$ & $\begin{array}{l}31 \\
35\end{array}$ & $\frac{17}{21}$ & 15.4 \\
\hline 556 & 31 & 28 & 36 & 8.6 \\
\hline 510 & 34 & 31 & 19 & 12.0 \\
\hline $\begin{array}{l}517 \\
535\end{array}$ & $\begin{array}{l}32 \\
37\end{array}$ & $\begin{array}{l}28 \\
33\end{array}$ & 20 & $\begin{array}{r}9.6 \\
14.6\end{array}$ \\
\hline 548 & 36 & 34 & 20 & 25.1 \\
\hline
\end{tabular}


Table 43. Length, width, thickness, and weight measurements of clams after three months of exposure. Date: $5 / 30 / 78$

\begin{tabular}{|c|c|c|c|c|}
\hline clam & Length $(\mathrm{mm})$ & Width $(m)$ & Thickness (mn) & Weight (grans) \\
\hline \multicolumn{5}{|c|}{ CONTROL } \\
\hline & 26 & 22 & 13 & 4.2 \\
\hline 31 & 40 & 35 & 21 & 16.9 \\
\hline 35 & 28 & 25 & 14 & 5.6 \\
\hline 49 & 40 & 35 & 22 & 17.7 \\
\hline 12 & 32 & 29 & 17 & 9.5 \\
\hline 33 & 32 & 29 & 17 & 9.3 \\
\hline 25 & 38 & 33 & 20 & 15.0 \\
\hline 23 & 36 & 32 & 19 & 12.7 \\
\hline \multicolumn{5}{|c|}{$6 \mathrm{Hg} / \mathrm{CPO}$} \\
\hline 702 & 28 & 25 & 15 & 6.3 \\
\hline 120 & 40 & 37 & 22 & 19.8 \\
\hline 144 & 25 & 23 & 13 & 4.4 \\
\hline 150 & 32 & 30 & 17 & 10.0 \\
\hline 113 & 26 & 23 & 13 & 5.0 \\
\hline 148 & 36 & 33 & 21 & 14.7 \\
\hline 115 & 34 & 31 & 16 & 9.7 \\
\hline 130 & 27 & 25 & 15 & 6.2 \\
\hline \multicolumn{5}{|c|}{$12 \mathrm{\mu g} / \mathrm{RCPO}$} \\
\hline 258 & 35 & 31 & 19 & 12.7 \\
\hline 220 & 33 & 30 & 37 & 9.6 \\
\hline 219 & 38 & 34 & 21 & 26.2 \\
\hline 229 & 35 & 32 & 29 & 12.7 \\
\hline 256 & 36 & 33 & 19 & 13.4 \\
\hline 242 & 35 & 32 & 20 & 13.7 \\
\hline 235 & 40 & 36 & 21 & 15.9 \\
\hline 239 & 37 & 34 & 21 & 15.9 \\
\hline \multicolumn{5}{|c|}{$25 \mu \mathrm{g} / \mathrm{R} \mathrm{CPO}$} \\
\hline 341 & 27 & 25 & 15 & 6.4 \\
\hline 350 & 41 & 36 & 22 & 19.4 \\
\hline 318 & 41 & 36 & 22 & 29.3 \\
\hline 349 & 36 & 32 & 19 & 12.1 \\
\hline 345. & 32 & 29 & 18 & 9.9 \\
\hline 357 & 42 & 39 & 23 & 22.2 \\
\hline 327 & 36 & 33 & 21 & 15.0 \\
\hline 330 & 37 & 34 & 21 & 15.7 \\
\hline \multicolumn{5}{|c|}{$50 \mu \mathrm{g} \& \mathrm{CPO}$} \\
\hline 458 & 37 & 33 & 20 & 14.2 \\
\hline 432 & 43 & 36 & 22 & 19.1 \\
\hline 429 & 39 & 37 & 20 & 17.5 \\
\hline 449 & 40 & 37 & 22 & 19.3 \\
\hline 446 & 31 & 28 & 17 & 8,4 \\
\hline 426 & 29 & 27 & 16 & 7.6 \\
\hline 408 & 31 & 28 & 17 & 8.7 \\
\hline 417 & 39 & 36 & 22 & 27.9 \\
\hline \multicolumn{5}{|c|}{$\frac{100 \mu g / R ~ C P O}{527}$} \\
\hline 527 & 38 & 34 & 19 & 14.4 \\
\hline 537 & 38 & 34 & 20 & 14.8 \\
\hline 524 & 43 & 38 & 22 & 19.5 \\
\hline 541 & 40 & 36 & 22 & 17.5 \\
\hline 503 & 35 & 32 & 19 & 12.8 \\
\hline 544 & 37 & 33 & 20 & 14.1 \\
\hline 534 & 38 & 35 & 22 & 17.1 \\
\hline 560 & 38 & 35 & 21 & 15.5 \\
\hline
\end{tabular}


Table 44. Length, width, thickness, and weight measurements of clams after four months of exposure. Date: 6/29/78

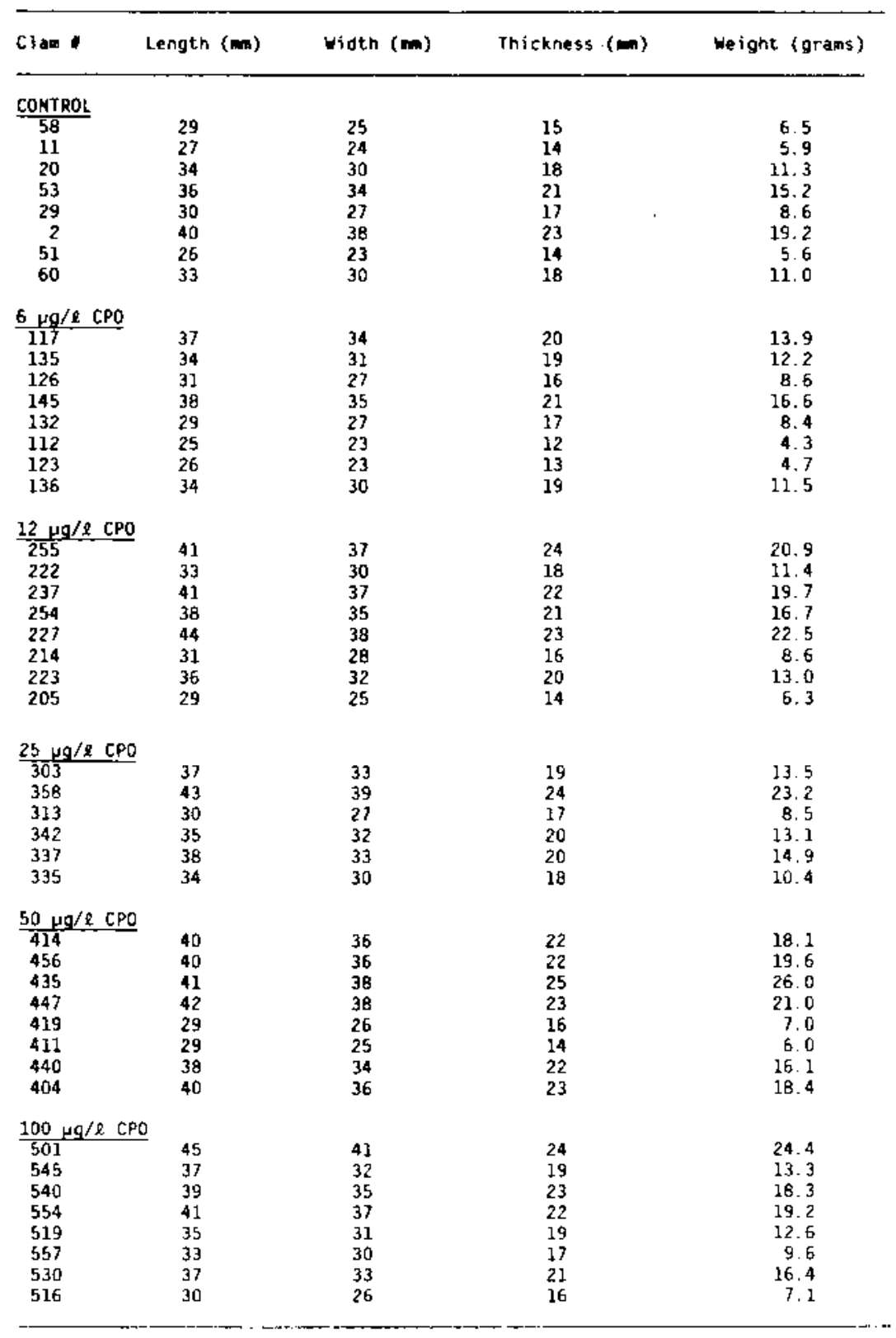


Table 45. Length, width, thickness, and weight measurements of clams after five months of exposure. Date: $8 / 1 / 78$

\begin{tabular}{|c|c|c|c|c|}
\hline Clam * & Length $(\mathrm{mm})$ & Width (mm) & Thickness $(\mathrm{mm})$ & Weight (grams) \\
\hline \multicolumn{5}{|c|}{ CONIROL } \\
\hline $\begin{array}{l}15 \\
47\end{array}$ & $\begin{array}{l}33 \\
33\end{array}$ & 29 & 17 & 9.6 \\
\hline 44 & 37 & 39 & 21 & 15.7 \\
\hline 48 & 32 & 29 & 18 & 9.8 \\
\hline 57 & 30 & 26 & 15 & 7.0 \\
\hline $\begin{array}{l}10 \\
59\end{array}$ & 30 & 28 & 17 & 8.8 \\
\hline $\begin{array}{l}59 \\
50\end{array}$ & $\begin{array}{l}27 \\
30\end{array}$ & 27 & $\begin{array}{l}15 \\
17\end{array}$ & $\begin{array}{l}6.2 \\
1.9\end{array}$ \\
\hline \multirow{2}{*}{\multicolumn{5}{|c|}{ E $\mathrm{pg} / \mathrm{CPO}$}} \\
\hline 141 & 30 & & 17 & B. 4 \\
\hline 139 & 35 & 32 & 20 & 13.9 \\
\hline 128 & 32 & 29 & 18 & 9.9 \\
\hline 124 & 29 & $\begin{array}{l}26 \\
27\end{array}$ & $\begin{array}{l}16 \\
15\end{array}$ & 6.8 \\
\hline 146 & 32 & 30 & 19 & 11.1 \\
\hline 111 & 29 & 25 & 15 & 6.2 \\
\hline 133 & 29 & 25 & 14 & 5.8 \\
\hline \multicolumn{5}{|c|}{$\frac{12}{250 / 2} / 2 \mathrm{CPO}$} \\
\hline 233 & 40 & $\begin{array}{l}35 \\
35\end{array}$ & 22 & $\begin{array}{l}17.0 \\
18.2\end{array}$ \\
\hline 230 & 39 & 35 & 21 & 17.3 \\
\hline 231 & 39 & 35 & 22 & 17.5 \\
\hline $\begin{array}{l}252 \\
202\end{array}$ & 34 & 31 & 19 & 12.2 \\
\hline $\begin{array}{l}202 \\
251\end{array}$ & $\begin{array}{l}29 \\
35\end{array}$ & $\begin{array}{l}25 \\
32\end{array}$ & 20 & $\begin{array}{l}6.5 \\
13.5\end{array}$ \\
\hline 253 & 38 & 35 & 22 & $\begin{array}{l}13.5 \\
17.0\end{array}$ \\
\hline \multirow{2}{*}{\multicolumn{5}{|c|}{$\frac{25 \mu \mathrm{\mu g} / \mathrm{R} C P 0}{20 \mathrm{~g}}$}} \\
\hline & 38 & 35 & 21 & 17.2 \\
\hline 319 & 38 & 35 & 22 & 17.4 \\
\hline 302 & 38 & 34 & 21 & 16.8 \\
\hline 347 & 41 & 37 & 22 & 19.3 \\
\hline 301 & 30 & 26 & 15 & 7.0 \\
\hline 307 & 34 & 32 & 19 & 12.6 \\
\hline 315 & 35 & 年 & $\begin{array}{l}16 \\
19\end{array}$ & 12.9 \\
\hline \multicolumn{5}{|c|}{$50 \mathrm{Hg} / \mathrm{l} C \mathrm{CPO}$} \\
\hline 415 & 33 & 30 & 18 & 11.1 \\
\hline 423 & 42 & 38 & 22 & 20.3 \\
\hline 412 & 41 & 36 & 23 & 18.6 \\
\hline $\begin{array}{l}436 \\
431\end{array}$ & ${ }_{35}^{41}$ & 37 & 22 & 18.7 \\
\hline 441 & 28 & $\frac{32}{25}$ & 14 & $\begin{array}{r}5.1 \\
5.8\end{array}$ \\
\hline 402 & 34 & 30 & 18 & $\begin{array}{r}7.8 \\
10.5\end{array}$ \\
\hline 443 & 34 & 31 & 18 & 10.9 \\
\hline \multicolumn{5}{|c|}{$\frac{100 \mu g / \ell}{500} \subset P O$} \\
\hline $\begin{array}{l}509 \\
546\end{array}$ & $\begin{array}{l}37 \\
42\end{array}$ & 38 & 23 & $\begin{array}{l}14.5 \\
20.2\end{array}$ \\
\hline 550 & 44 & 40 & 24 & 24.1 \\
\hline 520 & 37 & 33 & 21 & 15.0 \\
\hline $\begin{array}{l}536 \\
514\end{array}$ & 36 & 32 & 20 & 12.1 \\
\hline $\begin{array}{l}514 \\
523\end{array}$ & 34 & 31 & $\begin{array}{l}18 \\
18\end{array}$ & 11.0 \\
\hline 552 & 40 & 36 & $\frac{18}{22}$ & $\begin{array}{l}18.1 \\
18.9\end{array}$ \\
\hline
\end{tabular}


Table 46. Length, width, thickness, and weight measurements of clams after six months of exposure. Date: $9 / 5 / 78$

\begin{tabular}{|c|c|c|c|c|}
\hline $\mathrm{Clan}=$ & Length $(m)$ & Wiath $(\mathrm{m})$ & Thickness (m) & Weight (grans) \\
\hline \multicolumn{5}{|l|}{ CONTROL } \\
\hline$\overline{16}$ & 40 & 36 & 22 & 19.2 \\
\hline 32 & 40 & 37 & 22 & 20.1 \\
\hline 38 & 40 & 35 & 21 & 18.0 \\
\hline 40 & 36 & 32 & 18 & 12.0 \\
\hline 14 & 30 & 26 & 16 & 7.6 \\
\hline 5 & 32 & 30 & 18 & 10.5 \\
\hline 6 & 30 & 27 & 15 & 7.6 \\
\hline 45 & 31 & 29 & 27 & 9.6 \\
\hline \multicolumn{5}{|c|}{$6 \mu g / l \subset P O$} \\
\hline 105 & 38 & 35 & 21 & 16.4 \\
\hline 125 & 34 & 30 & 19 & 11.3 \\
\hline 151 & 35 & 31 & 18 & 11.2 \\
\hline 160 & 35 & 31 & 19 & 12.3 \\
\hline 131 & 28 & 25 & 14 & 6.2 \\
\hline 134 & 27 & 25 & 15 & 6.2 \\
\hline 107 & 30 & 28 & 16 & 8.3 \\
\hline 140 & 33 & 31 & 18 & 11.2 \\
\hline \multirow{2}{*}{\multicolumn{5}{|c|}{$\frac{12 \mu g / \ell C P O}{2}$}} \\
\hline & 36 & 32 & 19 & 12.8 \\
\hline 244 & 36 & 32 & 20 & 14.6 \\
\hline 245 & 37 & 32 & 19 & 13. 0 \\
\hline 210 & 33 & 30 & 19 & 11.7 \\
\hline 201 & 29 & 25 & 15 & 6.8 \\
\hline 204 & 31 & 28 & 16 & 8.0 \\
\hline 212 & 31 & 27 & 17 & 8.6 \\
\hline 260 & 38 & 34 & 21 & 26.3 \\
\hline \multicolumn{5}{|c|}{$\frac{25 \mu \mathrm{g} / \mathrm{R} \text { CPO }}{308}$} \\
\hline 308 & 43 & 38 & 22 & 21.3 \\
\hline 336 & 41 & 38 & 23 & 21.3 \\
\hline 339 & 40 & 36 & 22 & 18.6 \\
\hline 356 & 35 & 30 & 20 & 12.2 \\
\hline 314 & 34 & 30 & 17 & 9.9 \\
\hline 316 & 35 & 31 & 18 & 11.2 \\
\hline 353 & 34 & 31 & 18 & 10.4 \\
\hline 360 & 35 & 32 & 18 & 11.3 \\
\hline \multicolumn{5}{|c|}{$50 \mathrm{pg} / \ell \mathrm{CPO}$} \\
\hline $\begin{array}{l}406 \\
410\end{array}$ & $\begin{array}{l}38 \\
41\end{array}$ & $\begin{array}{l}33 \\
37\end{array}$ & $\begin{array}{l}20 \\
27\end{array}$ & $\begin{array}{l}14.9 \\
19.8\end{array}$ \\
\hline $\begin{array}{l}410 \\
430\end{array}$ & $\begin{array}{l}41 \\
37\end{array}$ & 34 & 20 & $\begin{array}{l}19.8 \\
14.2\end{array}$ \\
\hline 439 & 40 & .37 & 22 & 19.0 \\
\hline 401 & 26 & 23 & 13 & 5.0 \\
\hline 413 & 33 & 30 & 19 & 14.2 \\
\hline 457 & 35 & 30 & 18 & 11.9 \\
\hline \multicolumn{5}{|c|}{$\frac{100 \mu g / 2 C P O}{606}$} \\
\hline 506 & 38 & 34 & 19 & 14,1 \\
\hline 525 & 42 & 40 & 23 & 21.7 \\
\hline 528 & 43 & 40 & 24 & 24.8 \\
\hline 558 & $3 \mathrm{e}$ & 35 & 21 & 15.7 \\
\hline 507 & 38 & 34 & 21 & 34.9 \\
\hline 511 & 30 & 26 & 15 & 6.4 \\
\hline 513 & 32 & 29 & 17 & 9.6 \\
\hline 521 & 32 & 30 & 19 & 11.0 \\
\hline
\end{tabular}


Table 47. Length, width, thickness, and weight measurements of clams after eight months of exposure. Date: 11/8/78

\begin{tabular}{|c|c|c|c|c|}
\hline Clam & Length $(\mathrm{mm})$ & Vidth (mm) & Thickness (mon) & Weight (grams) \\
\hline \multicolumn{5}{|c|}{ CONTROL } \\
\hline 24 & 30 & 27 & 15 & 7.3 \\
\hline 7 & 28 & 24 & 1.4 & 6.1 \\
\hline 19 & 34 & 31 & 19 & 12.7 \\
\hline 43 & 36 & 32 & 19 & 12.8 \\
\hline 37 & 31 & 28 & 17 & 8.9 \\
\hline 34 & 33 & 30 & 18 & 11.6 \\
\hline 54 & 29 & 26 & 15 & 7.1 \\
\hline 13 & 29 & 26 & 16 & 7.6 \\
\hline 52 & 34 & 31 & 20 & 11.9 \\
\hline 4 & 27 & 25 & 15 & 6.2 \\
\hline 56 & 36 & 33 & 20 & 13.9 \\
\hline \multicolumn{5}{|c|}{$6 \mathrm{~kg} / 2 \mathrm{CPO}$} \\
\hline 154 & 37 & 32 & 20 & 14.2 \\
\hline 153 & 36 & 32 & 20 & 13.7 \\
\hline 127 & 26 & 23 & 15 & 5.5 \\
\hline 157 & 39 & 36 & 21 & 26.9 \\
\hline 137 & 32 & 29 & 17 & 9.9 \\
\hline 142 & 30 & 27 & 16 & 7.8 \\
\hline 101 & 30 & 28 & 27 & 9.6 \\
\hline 129 & 25 & 22 & 13 & 4.4 \\
\hline 121 & 36 & 32 & 18 & 12.9 \\
\hline 149 & 35 & 31 & 18 & 11.5 \\
\hline \multicolumn{5}{|c|}{$12 \mathrm{Hg} / \mathrm{CPO}$} \\
\hline 238 & 33 & 30 & 18 & 11.7 \\
\hline 221 & 31 & 29 & 17 & 9.4 \\
\hline 243 & 34 & 31 & 17 & 10.7 \\
\hline 213 & 28 & 26 & 14 & 5.7 \\
\hline 246 & 41 & 36 & 20 & 18.7 \\
\hline 215 & 31 & 27 & 16 & 8.4 \\
\hline 224 & 35 & 31 & 18 & 12.2 \\
\hline \multicolumn{5}{|c|}{$25 \mathrm{HQ} / \mathrm{R} C \mathrm{CPO}$} \\
\hline 351 & 30 & 26 & 16 & 8. 0 \\
\hline 338 & 38 & 34 & 21 & 25.2 \\
\hline 322 & 30 & 26 & 1.5 & 7.2 \\
\hline 324 & 34 & 30 & 18 & 10.8 \\
\hline 325 & 32 & 29 & 17 & 9.7 \\
\hline 354 & 37 & 33 & 21 & 15. 8 \\
\hline 343 & 38 & 34 & 21 & 16.1 \\
\hline 328 & 41 & 37 & 22 & 19.0 \\
\hline 312 & 28 & 26 & 15 & 6.9 \\
\hline 326 & 32 & 29 & 18 & 10.0 \\
\hline \multicolumn{5}{|c|}{$50 \mathrm{Hg} / \ell \subset P O$} \\
\hline 409 & 34 & 30 & 17 & 10.6 \\
\hline 442 & 26 & 23 & 14 & 5.0 \\
\hline 403 & 29 & 26 & I6 & 8.0 \\
\hline 420 & 32 & 29 & 17 & 9.3 \\
\hline 433 & 38 & 35 & 21 & 16.8 \\
\hline 437 & 27 & 25 & 14 & 5.9 \\
\hline 451 & 32 & 28 & 17 & B. 9 \\
\hline \multicolumn{5}{|c|}{100 上g/E CPO } \\
\hline 508 & 37 & 32 & 20 & 13.7 \\
\hline 549 & 35 & 33 & 21 & 13.9 \\
\hline 502 & 39 & 36 & 22 & 18.5 \\
\hline 543 & 32 & 28 & 18 & 9.5 \\
\hline 532 & 42 & 37 & 22 & 19.6 \\
\hline 518 & 31 & 28 & 17 & 8. 5 \\
\hline 531 & 34 & 31 & 18 & 11.5 \\
\hline 559 & 36 & 32 & 19 & 13.3 \\
\hline 551 & 35 & 31 & 18 & 11.3 \\
\hline
\end{tabular}


Table 48. Measured CPO concentrations $(\mu \mathrm{g} / \ell)$ in exposure tanks.



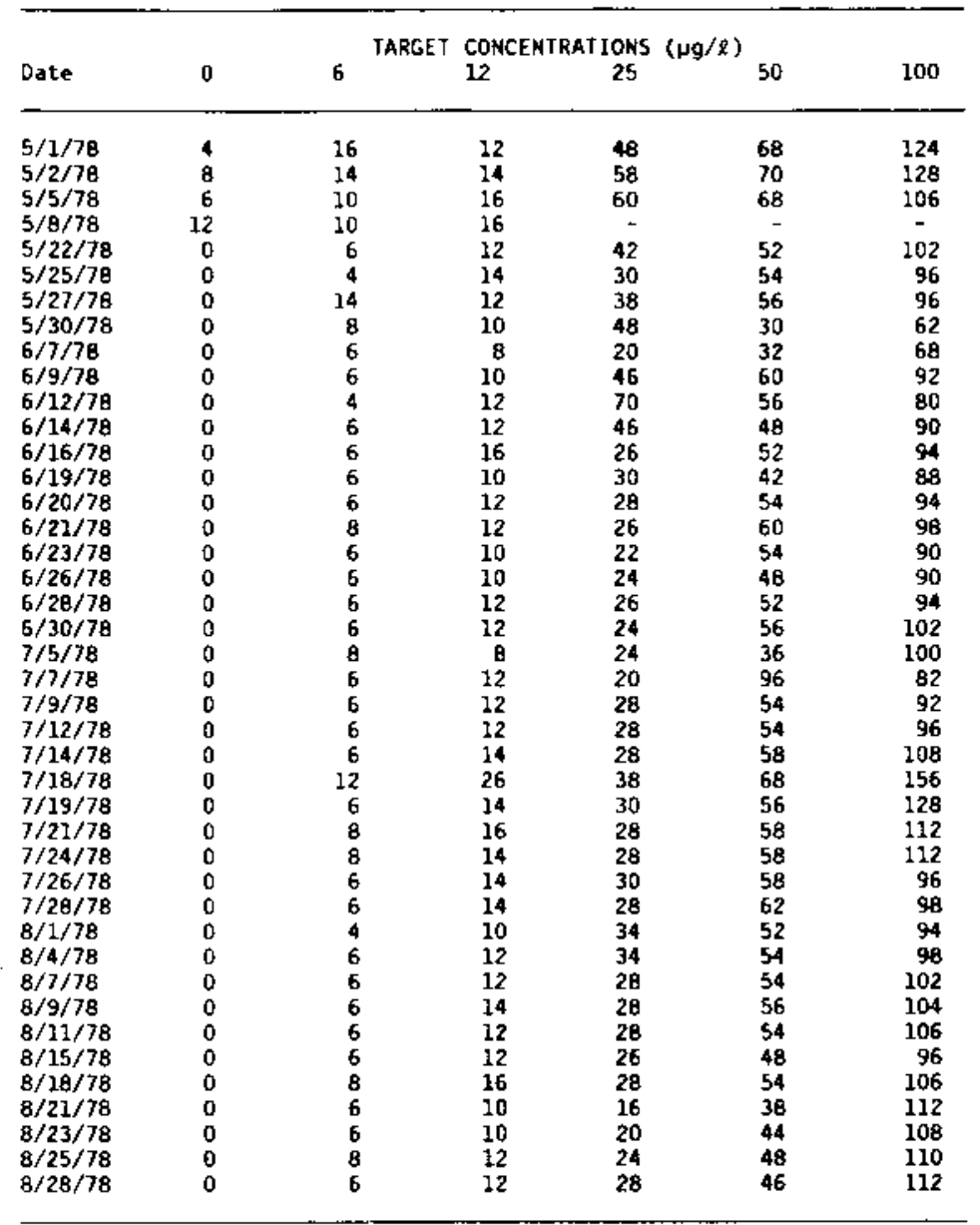


Table 49. Histopathological descriptions of Protothaca staminea from Sequim Bay, Washington.

$R=$ ripe gonad; $P S=$ portially spamed gonad; $S=$ spent gonad; $\delta=$ male; $q$ = female

\begin{tabular}{|c|c|c|}
\hline $\begin{array}{l}\text { Identi- } \\
\text { fication }\end{array}$ & $\begin{array}{l}\text { Specimen } \\
\text { Number }\end{array}$ & Description \\
\hline \multirow{10}{*}{$\begin{array}{l}\text { Initial } \\
\text { Sanple }\end{array}$} & $79-75$ & $5^{9}$ \\
\hline & $79-76$ & Ró; sone metaplasia of digestive gland \\
\hline & $79-77$ & PST \\
\hline & $79-78$ & PSP; some necrosis of stonach; some metaplasia of digestive tubules \\
\hline & $79-79$ & PSo'; metazoan parasite in gonad \\
\hline & $79-80$ & PSf; gregarine-like parasite in mantle; some necrotic gill epithelium \\
\hline & $79-81$ & $\begin{array}{l}\text { PSP; metazoan parasite in kidmey; gregarine-like parasite in gill; some necrosis in } \\
\text { stomach }\end{array}$ \\
\hline & $79-82$ & PSơ; generally necrotic \\
\hline & $79-83$ & PSE; generally necrotic \\
\hline & $79-84$ & PSf: generatly necrotic \\
\hline
\end{tabular}


Tab1e 50. Histopathological descriptions of Protothaca staminea used as controls in Jank No. 1 of Chlorine Bioassay Study.

$L A=$ late active gonad; $R=$ ripe gonad: $P S=$ partially spawned gonad; $S=$ spent gonad; $\delta$ = male; $q=$ female

\begin{tabular}{|c|c|c|}
\hline $\begin{array}{l}\text { Exposure } \\
\text { Tine }\end{array}$ & $\begin{array}{l}\text { Specimen } \\
\text { Nuber }\end{array}$ & Description \\
\hline \multirow{4}{*}{$\begin{array}{l}\text { 1-Month } \\
\text { Expasure }\end{array}$} & $79-85$ & LAd \\
\hline & $79-86$ & PSF; portions of gill epithel tun necrotic \\
\hline & $79-87$ & Ró; Detazoan parasite in kidney; necrosis of portions of gill, stansch, intestine \\
\hline & $79-88$ & PSF; gregarine parasite; necrosis of portions of stomach. digestive tubules \\
\hline \multirow{4}{*}{$\begin{array}{l}\text { 2-Honth } \\
\text { Exposure }\end{array}$} & $79-89$ & PSP \\
\hline & $79-90$ & PSF; metazoan parasite in kidney \\
\hline & $79-91$ & $\begin{array}{l}\text { Ró; metazoan parasite in digestive gland; slight leukocytic inf } \sharp 1 \text { tration; some necrosis } \\
\text { of digestive giand near parasite cysts }\end{array}$ \\
\hline & $79-92$ & Rq; metazoan present in kloney \\
\hline \multirow{4}{*}{$\begin{array}{l}\text { 3-Month } \\
\text { Exposure }\end{array}$} & $79-93$ & PS6 \\
\hline & $79-94$ & PS? \\
\hline & $79-95$ & $\begin{array}{l}\text { PSd: unidentified organism in kidney; metazoan parasite in kidney; leukocytic } \\
\text { infiltration in area of cysts }\end{array}$ \\
\hline & $79-96$ & PSP \\
\hline \multirow{4}{*}{$\begin{array}{l}\text { 4-Month } \\
\text { Exposure }\end{array}$} & $79-97$ & Rat \\
\hline & $79-98$ & PSf; necrotic tissue in kioney, stanach; some digestive tubules metaplasia \\
\hline & $79-99$ & LAd; gregarine parasite in gill, some leukocytic infiltration \\
\hline & $79-100$ & PSf; abscess in kidney; some necrosis of stanach epithelium \\
\hline \multirow[t]{3}{*}{$\begin{array}{l}\text { 5-Month } \\
\text { Exposure }\end{array}$} & $79+101$ & PSó:: some necrosis of digestive tubules; numerous abscesses \\
\hline & $79-102$ & PSó; same necrosis and metapiasta of digestive tubules \\
\hline & $79-103$ & PSd; same necrosis and metaplasid of digestive tubules \\
\hline \multirow{4}{*}{$\begin{array}{l}\text { 6-Month } \\
\text { Exposure }\end{array}$} & $79-105$ & PSF; occasional metaplastic digestive tubules \\
\hline & $79-106$ & PSF; gregarine parasite in gill mantle; metazoan parasite in kldney \\
\hline & $79-107$ & PSF; metazoan parasite in kichey; some metaplasia of digestive tubules \\
\hline & $79-108$ & Rd; gregarine parasite in gill \\
\hline
\end{tabular}


Table 51. Histopathological descriptions of Protothaca staminea exposed to $6 \mathrm{ppb}$ of chlorine in Tank No. 2 of Chlorine Bioassay Study.

$L A=$ late active gonad; $R=$ ripe gonad; $P S=$ partially spamed gonad; $S=$ spent gonad; $6=$ male; $f=$ female

\begin{tabular}{|c|c|c|}
\hline $\begin{array}{l}\text { Exposure } \\
\text { Time }\end{array}$ & $\begin{array}{l}\text { Specionen } \\
\text { Number }\end{array}$ & Description \\
\hline \multirow[t]{4}{*}{$\begin{array}{l}\text { I-Month } \\
\text { Exposure }\end{array}$} & $79-109$ & $\begin{array}{l}\text { Lod; some necrosis in gill; some metaplasia of digestive tubules; autolysis of } \\
\text { Leydig celis }\end{array}$ \\
\hline & $79-110$ & Lat; autolysts of Leydig cells; large eosinophilic leukocytes in kidney \\
\hline & $79-111$ & $\begin{array}{l}\text { PSF; metazoan parasite in kidney; abscess in kidney filled with large eosinophilic } \\
\text { leukocytes; some necrosis of intestine }\end{array}$ \\
\hline & $79-112$ & $\begin{array}{l}\text { PSP; metazoan parasite in kidney; abscesses in kidney; large eosinophillc leukocytes; } \\
\text { metaplastic digestive tubules; some necrosis of stomach epithellum }\end{array}$ \\
\hline \multirow{4}{*}{$\begin{array}{l}\text { 2-Honth } \\
\text { Exposure }\end{array}$} & $79-113$ & s? \\
\hline & $79-114$ & PSP \\
\hline & $79-115$ & $\operatorname{Rd}$ \\
\hline & $79-116$ & $\begin{array}{l}\text { LAd; metazaan parasite in kidney; leukocytic infiltration; cluster of eosinophilic } \\
\text { leulocytes }\end{array}$ \\
\hline \multirow[t]{4}{*}{$\begin{array}{l}\text { J-Menth } \\
\text { Exposure }\end{array}$} & $79 \cdot 117$ & LAd \\
\hline & $79-118$ & PSó \\
\hline & $79-119$ & PS? \\
\hline & $79-120$ & $\begin{array}{l}\text { Ros; unidentlfied omanjsm in gill; some leukocytic infittration; small abscess in } \\
\text { nantle }\end{array}$ \\
\hline \multirow[t]{4}{*}{$\begin{array}{l}\text { 4-Month } \\
\text { Exposure }\end{array}$} & $79-121$ & Sf; undentified organism in gill; some leukocytic infiltration \\
\hline & $79-122$ & Sf; uldentified organism(s) in glil, kidney; metazoan parasite in kidney \\
\hline & $79-123$ & $\begin{array}{l}\text { Sf; metazoan paraste in kidney; unidentified organisms; sone metoplasia of digestive } \\
\text { tubules; yacuolization of stomach epitheilium }\end{array}$ \\
\hline & $79-124$ & $\begin{array}{l}\text { Po: gregarine-ifke parasite in foot; some netaplasia of digestive tubules; } \\
\text { vacuolization of stamach epithelitum }\end{array}$ \\
\hline \multirow[t]{4}{*}{$\begin{array}{l}\text { 5-Honth } \\
\text { Expos ure }\end{array}$} & $79-125$ & $\begin{array}{l}\text { Só; metazoan parasite in kidney (heavy infection); general leulocytosis; autolysis } \\
\text { of Leydlg cells; scone metaplasio of digestive tubules }\end{array}$ \\
\hline & $79-126$ & PSor; some necros 15 of stonach, kidney tubules; autolysis of Leydig cells \\
\hline & $79-127$ & $\begin{array}{l}\text { 57; metazoan parastt in kidney; leukocytic inflitration into kidmey area; necrotic } \\
\text { kidney tubules; vacuolization of stamach and intestinal eplthelium }\end{array}$ \\
\hline & $78-128$ & $\begin{array}{l}\text { Sd; metazoan parasite in kidney; leukocytic infiltration into kidney; soose } \\
\text { eosinophilic leukocytes }\end{array}$ \\
\hline \multirow[t]{4}{*}{$\begin{array}{l}\text { 6-Month } \\
\text { Exposure }\end{array}$} & $79-129$ & SP; metazoan parasite in kidney; leukocytic infiltration; some necrosts \\
\hline & $79 \cdot 130$ & $\begin{array}{l}\text { RP; metuzoan parastue in kidney; considerable necrosis of digestive tubules and } \\
\text { kidney tubules; necrotic areas of stomacb intestinal opithelfum }\end{array}$ \\
\hline & $79-131$ & $\begin{array}{l}\text { P5f; necrosis of kidney tubules; autolysis of Leydig cells; soue necrosis and } \\
\text { metaplasia of digestive tubule epitheifum }\end{array}$ \\
\hline & $79-132$ & $\begin{array}{l}\text { Sf; netazoan parasite in kioney with some enlarged eosinophilic leukocytes: } \\
\text { necrotic areas of stanach eptthelfum }\end{array}$ \\
\hline
\end{tabular}


Table 52. Histopathological description of Protothaca staminea exposed to $12 \mathrm{Fpb}$ chlorine in Tank No. 3 of Chlorine Bioassay Study.

LA = late active gonad; $R=$ ripe gonad; $P S=$ partially spamed gonad; $S=$ spent gonad, $\delta=$ male; $q=$ fenale

\begin{tabular}{|c|c|c|}
\hline $\begin{array}{l}\text { Exposure } \\
\text { Tiane }\end{array}$ & $\begin{array}{l}\text { Specimen } \\
\text { Number }\end{array}$ & Description \\
\hline \multirow[t]{4}{*}{$\begin{array}{l}\text { l-Month } \\
\text { Exposure }\end{array}$} & $79-133$ & LAo:; all tissues generally necrotic, clan may have been dead \\
\hline & $79-134$ & LAd'; same as above \\
\hline & $79-135$ & PS?: same as above \\
\hline & $79-136$ & PSP; sane as above \\
\hline \multirow{4}{*}{$\begin{array}{l}\text { 2-Honth } \\
\text { Exposure }\end{array}$} & $79-137$ & PSF; gregar Ine-iłke parasite in gill \\
\hline & $79-138$ & psf; sume necrosts of g111 and digestive tubutes; autolysis of leydig cells \\
\hline & $79-139$ & 957; necrosis, metaplasia of digestive tubules; autolys is of Leydig cells \\
\hline & $79-140$ & Ró; metazoan parasite in gonad \\
\hline \multirow{4}{*}{$\begin{array}{l}\text { 3-Honth } \\
\text { Exposure }\end{array}$} & $79-141$ & P56; metazoan parasite_in kidney: leukocytic infiltration into kidney; gill \\
\hline & $79-142$ & P57; large abscess in gonod \\
\hline & $79 \cdot 143$ & Ró \\
\hline & $79-144$ & $\mathrm{R} \delta$ \\
\hline \multirow[t]{4}{*}{$\begin{array}{l}\text { 4-Month } \\
\text { Exposure }\end{array}$} & $79-145$ & $\begin{array}{l}\text { PSd; gregarine-like parasite in gill; metazoan parasite in kidney; leukocytic } \\
\text { infiltration in viscera around cysts }\end{array}$ \\
\hline & $79-146$ & PSó; basophilic granular materíal in gills \\
\hline & $79-147$ & $\begin{array}{l}\text { PSP; gregarine-like parasite in gill; some metaphasia of digestive tubules; some } \\
\text { necrosis }\end{array}$ \\
\hline & $79-149$ & $\begin{array}{l}\text { Rq; metazoan parasite in kidney; metaplasia of digestive tubules; some necrosis in } \\
\text { kidney, gill, digestive tubules, stamach and intestinal eptothelium }\end{array}$ \\
\hline \multirow{4}{*}{$\begin{array}{l}\text { 5-Month } \\
\text { Exposure }\end{array}$} & $79-149$ & So: metazoan parasite (heavy infection) in kidney; leukocytic infijtration into kidne \\
\hline & $79-150$ & PSf: metazoan parasite in kidney; some metaplasia and necrosis of digestive tubules \\
\hline & $79-15 T$ & PSf; generalized leukocytos is \\
\hline & $79-152$ & so; some necrosis of intestinal ept the lium, stamach, digestive tubules \\
\hline \multirow{4}{*}{$\begin{array}{l}\text { 6-Month } \\
\text { Exposure }\end{array}$} & $79-153$ & Sf; scme metapiasia, necrosis of digestive tubules; autolysis of Leydig cells \\
\hline & $79-154$ & PSP; some metaplasla, necrosis of digestive tubules \\
\hline & $79-155$ & Sf: metazoan parasfte in kidney, leukocytosis around cysts \\
\hline & $79-i 56$ & $\begin{array}{l}\text { - no gonad; general leukocytosis, especially in digestive gland; fíbrous deposition } \\
\text { in digestive gland be tween tubules }\end{array}$ \\
\hline
\end{tabular}


Table 53. Histopathological description of Protothaca staminea exposed to $25 \mathrm{ppb}$ chlorine in Tank No. 4 of Chlorine Bioassay Study.

$L A=$ late active gonad; $R=$ ripe ganad; $P S=$ partially spamed gonad; $S=$ spent gonad; $\sigma=$ male; $q=$ female $E A=$ early active gonad




Table 54. Histopathological descriptions of Protothaca staminea exposed to $50 \mathrm{ppb}$ chlorine in Tank No. 5. of Chiorine Bioassay Study.

$L A=$ late active ganad; $R=$ ripe gonad; $P A=$ partlally spamed gonad; $S=$ spent gonad; $f=$ male; $f=$ fenale

\begin{tabular}{|c|c|}
\hline $\begin{array}{c}\text { Exposure } \\
\text { Thine }\end{array}$ & $\begin{array}{l}\text { Specimen } \\
\text { Kumber }\end{array}$ \\
\hline \multirow{4}{*}{$\begin{array}{l}\text { 1-Month } \\
\text { Exposure }\end{array}$} & $79-181$ \\
\hline & $79-182$ \\
\hline & 79.183 \\
\hline & $79-184$ \\
\hline \multirow{4}{*}{$\begin{array}{l}\text { 2-Month } \\
\text { Exposure }\end{array}$} & $79-185$ \\
\hline & $79-186$ \\
\hline & 79.187 \\
\hline & $79-188$ \\
\hline \multirow[t]{4}{*}{$\begin{array}{l}\text { 3-Month } \\
\text { Exposure }\end{array}$} & $79-189$ \\
\hline & $79-190$ \\
\hline & $79-191$ \\
\hline & $79-192$ \\
\hline \multirow[t]{4}{*}{$\begin{array}{l}\text { 4-Month } \\
\text { Exposure }\end{array}$} & $79-193$ \\
\hline & $79-194$ \\
\hline & $79-195$ \\
\hline & $79-196$ \\
\hline \multirow[t]{4}{*}{$\begin{array}{l}\text { 5-Month } \\
\text { Exposure }\end{array}$} & $79-197$ \\
\hline & $79-198$ \\
\hline & $79-199$ \\
\hline & $79-200$ \\
\hline \multirow[t]{4}{*}{$\begin{array}{l}\text { 6-Month } \\
\text { Exposure }\end{array}$} & $79-201$ \\
\hline & $79-202$ \\
\hline & $79-203$ \\
\hline & $79-204$ \\
\hline
\end{tabular}


Table 55. Histopathological descriptions of Protothaca staminea exposed to $100 \mathrm{ppb}$ chlorine in Tank No. 6 of Chlorine Bioassay Study.

$L A=$ late active gonad; $R=$ ripe gonad; $P S=$ partially spamed gonad; $S=$ spent gonad; $\sigma=$ male $;=$ female

\begin{tabular}{|c|c|c|}
\hline $\begin{array}{l}\text { Exposure } \\
\text { Trime }\end{array}$ & $\begin{array}{l}\text { Specinen } \\
\text { Mumber }\end{array}$ & Description \\
\hline \multirow[t]{4}{*}{$\begin{array}{l}\text { 1-Month } \\
\text { Exposure }\end{array}$} & $79-205$ & $\begin{array}{l}\text { Ró; metazoan parasite in kidney, leukocytosis; large masses of basophilic granular } \\
\text { material in gillis; necrosis of iarge portion of gill; leukocytosis }\end{array}$ \\
\hline & $79-206$ & LAd; general necrosis \\
\hline & $79-207$ & LAd; general necrosis \\
\hline & $79-208$ & Rot; metazoan parasite in kidney; general necrosis \\
\hline \multirow[t]{4}{*}{$\begin{array}{l}\text { 2- Month } \\
\text { Exposure }\end{array}$} & $79-209$ & $\begin{array}{l}\text { Af; leukocytic infil tration into gills, gonad, digestive area: vacuolization of } \\
\text { stamach, Intestinal epl thelfun with some necrosis }\end{array}$ \\
\hline & $79-210$ & Sf; autolysis of Leydig cells; some leukocytic accumulation around stomach \\
\hline & $79-211$ & $\begin{array}{l}\text { PSf; metazoan parasite in gonad: autolysis of Leydig cells; necrosis of digestive } \\
\text { tubules, portions of gills; vacuolization and some necrosis of intestinal epithelifum }\end{array}$ \\
\hline & $79-212$ & $\begin{array}{l}\text { LAd autolysis of Leydig tissues; leukocytos is of gills; vacuolization of digestive } \\
\text { tube epithelium, same necrosis }\end{array}$ \\
\hline \multirow[t]{4}{*}{$\begin{array}{l}\text { 3- Month } \\
\text { Exposure }\end{array}$} & $79-213$ & $\begin{array}{l}\text { PS6; autolysis of Leydig cells; necrosis, metaplasia of digestive tubules; necrosis } \\
\text { of stomach and intestinal epithelium }\end{array}$ \\
\hline & $79-214$ & $\begin{array}{l}\text { Lad: autolysis of Leydig cells; vacuolization of stomach epithelium; some necros is } \\
\text { of digestive tubules; intestinal epithelium }\end{array}$ \\
\hline & $79-215$ & 5f; abscess on gilt; small anount of necrosis, metaplasia of digestive tubules \\
\hline & $79-216$ & $\begin{array}{l}\text { PSP: necrotic areas of digestive gland; vacuolization, same necrosis of stomach } \\
\text { epithellim }\end{array}$ \\
\hline \multirow{4}{*}{$\begin{array}{l}\text { 4-Math } \\
\text { Exposure }\end{array}$} & $79-217$ & LAd; metazoan parasite in kidney; slight leukocytosis in area of parasite \\
\hline & $79-278$ & LAG" \\
\hline & $79-219$ & Sf; metazoan parasite in kidney; metaplasia, necrosis of digestive tubules \\
\hline & $79-220$ & PSf: vaciolization of stamach and intestinal epithelium, some necrosis \\
\hline \multirow{4}{*}{$\begin{array}{l}\text { 5-Month } \\
\text { Exposure }\end{array}$} & $79-221$ & 57; autolysis of Leydig cells \\
\hline & $79-222$ & PSf; vacuolization of intestinal epithelium \\
\hline & $79-223$ & s? \\
\hline & $79-224$ & $\${ }^{f}$; vacuolization of stomach, intestinal ept thelfum \\
\hline \multirow[t]{4}{*}{$\begin{array}{l}\text { 6-Honth } \\
\text { Exposure }\end{array}$} & $79 \cdot 225$ & $\begin{array}{l}\text { sf; autolysis of Leydig celis; generai leukocytosis; metapiasia; necrosis of stamach. } \\
\text { intestinal epi thelifm, kidney }\end{array}$ \\
\hline & $79-226$ & $\begin{array}{l}\text { PSF; gilis necrotic; vacuolization of stanach, Intestinal epitheliuni vacuolization, } \\
\text { some necrosis of digestive tubules }\end{array}$ \\
\hline & $79-227$ & $\begin{array}{l}\text { Ró; necrotic areas along gills; digestive tubules almost completely necrotic; autolysi } \\
\text { of Leydig cells; necrosis of stamach, intestine }\end{array}$ \\
\hline & $79-228$ & $\begin{array}{l}\text { Lad; vacuol ization of intestinal epithelium, some necrosis; general leukocytos is; } \\
\text { necrotic areas of gifl }\end{array}$ \\
\hline
\end{tabular}


Table 56. Results of histopathological examination of injtial sample of Protothaca staminea collected from Sequim Bay, Washington in March, 1978.

$R=$ rfpe gonad; PS= partially spamed gonad; $S$ - spent gonad; M = Metazoan parasite; G = gregarine-like parasite; $\sigma=$ male; $q=$ female

\begin{tabular}{|c|c|c|c|c|c|c|}
\hline $\begin{array}{l}\text { Specimen } \\
\text { Number }\end{array}$ & $\begin{array}{l}\text { Gonad } \\
\text { Condition }\end{array}$ & Parasite & Leukocytosis & Metaplasia & Necrosis & Other \\
\hline $79-75$ & 57 & & & $\begin{array}{l}\text { some: digestive } \\
\text { tubules }\end{array}$ & & \\
\hline $79-76$ & $\mathrm{R} \sigma^{*}$ & & & $\begin{array}{l}\text { some; digestive } \\
\text { tubules }\end{array}$ & some; stomach & \\
\hline $79-77$ & $\mathrm{PS}^{\mathrm{P}}$ & $M$; gonad & & & & \\
\hline $79-80$ & PSF & $G_{i}$ mantie & & & some; gill & \\
\hline $79-81$ & PS\& & $M ;$ kidney & & & some; stomach & \\
\hline $79-82$ & $P 5 \sigma^{\circ}$ & & & & genera? & \\
\hline $79+83$ & PSó" & & & & general & \\
\hline $79-84$ & psf & & & & general & \\
\hline
\end{tabular}




\section{Table 57. Summary of histological descriptions of the littleneck clam, Protothaca staminea, exposed to various amounts of chlorine for one month.}

$L A=$ late active gonad; $R=$ ripe gonad; PS - partially spawned gonad; 5 = spent gonad; M - metazoan parasite; G = gregarine-like parasite: $d=$ male; $F=$ fatale






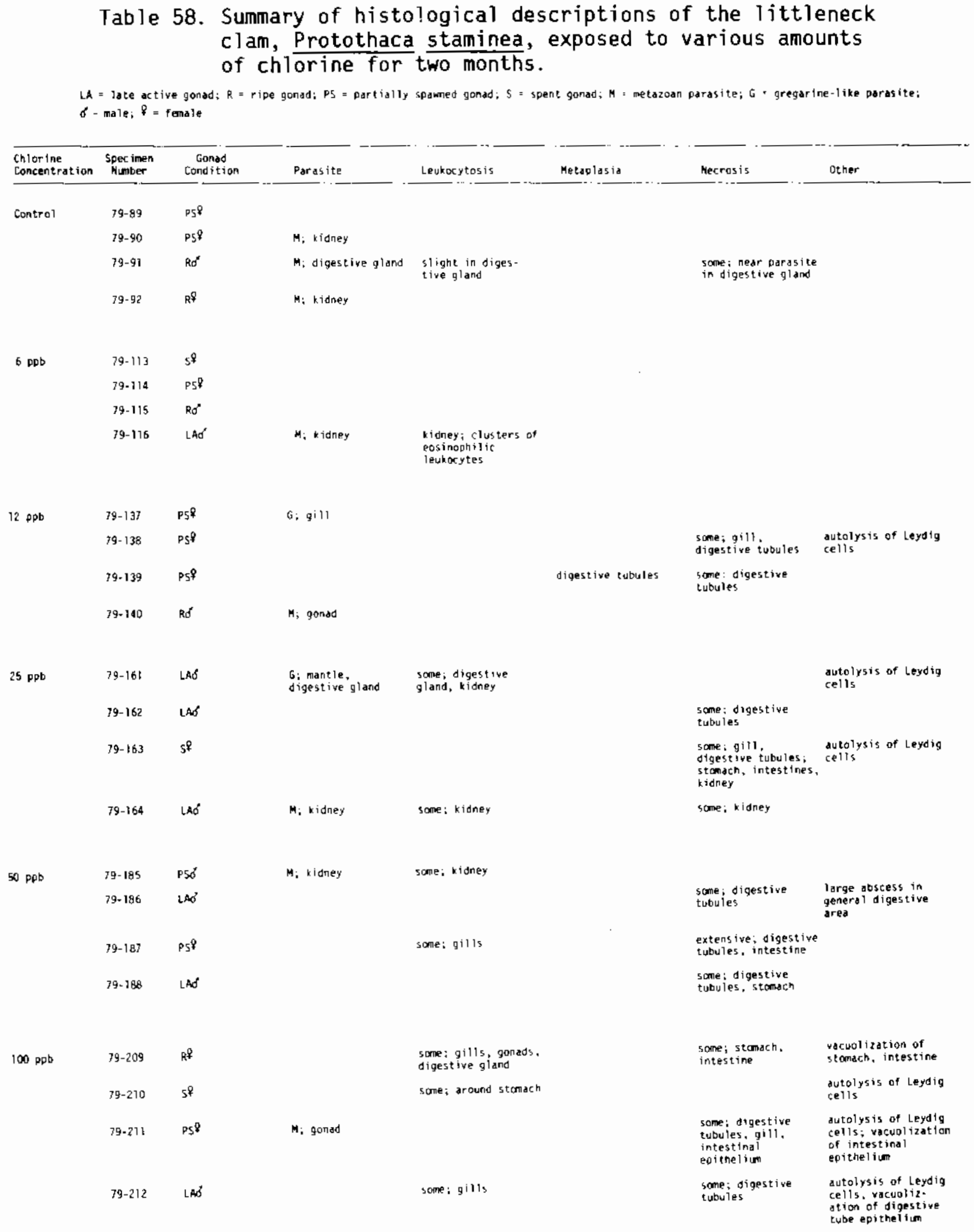




\section{Table 59. Summary of histological descriptions of the littleneck clam, Protothaca staminea, exposed to various amounts of chlorine for three months.}

LA = late active gonad; $R=$ ripe gonad: $P S=$ partially spawned gonad; $S=$ spent gondd; $M=$ metazoan parasite; $f=$ gregarine-Jike parasite: $d^{*}=$ male: $7=$ female; $E A=$ early active gonad

\begin{tabular}{|c|c|c|c|c|c|c|}
\hline $\begin{array}{l}\text { Chlorine } \\
\text { Concentration }\end{array}$ & $\begin{array}{l}\text { Specimen } \\
\text { Number }\end{array}$ & $\begin{array}{c}\text { Gonad } \\
\text { Condition }\end{array}$ & Parasite & leukorytosis Metaplasid & Necrosis & Other \\
\hline \multirow[t]{4}{*}{ Controì } & 79.93 & p5d & & & & \\
\hline & $79-94$ & PSP & & & & \\
\hline & $79-95$ & PSo & $\begin{array}{l}\text { M; kidney } \\
\text { Unident ified: } \\
\text { kidney }\end{array}$ & some; kidney & & \\
\hline & $79-96$ & $\mathrm{P}^{\circ} \mathrm{P}$ & & & & \\
\hline \multirow[t]{4}{*}{$6 \mathrm{ppb}$} & $79-117$ & LAó & & & & \\
\hline & $79-118$ & $\operatorname{PSo} \sigma^{x}$ & & & & \\
\hline & $79-119$ & 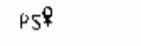 & & & & \\
\hline & $79-120$ & $\mathrm{R} \delta$ & $\begin{array}{l}\text { Unidentified: } \\
\text { gill }\end{array}$ & soine; gוl & & $\begin{array}{l}\text { small abscess in } \\
\text { mantle }\end{array}$ \\
\hline \multirow[t]{4}{*}{$12 \mathrm{ppb}$} & $79-141$ & psd́ & $M ;$ *idney & some; kidney. gill & & \\
\hline & $79-142$ & PSP & & & & $\begin{array}{l}\text { large abscess in } \\
\text { gland }\end{array}$ \\
\hline & $79-143$ & $\mathrm{R} \sigma$ & & & & \\
\hline & $79-144$ & $R \sigma^{*}$ & & & & \\
\hline
\end{tabular}

\begin{tabular}{|c|c|c|c|c|c|c|c|}
\hline \multirow[t]{4}{*}{$25 \mathrm{ppb}$} & $79-165$ & EAO & & & & & \\
\hline & $79-166$ & PSo & $M$; kidney & & & & \\
\hline & $79-167$ & \$? & & & $\begin{array}{l}\text { somf: digentive } \\
\text { tubules }\end{array}$ & \multicolumn{2}{|l|}{$\begin{array}{l}\text { some; digertive } \\
\text { tubules, intestinal } \\
\text { epithel ium }\end{array}$} \\
\hline & $79-168$ & $\mathrm{PS}^{9}$ & M: kidney & & & \multicolumn{2}{|l|}{$\begin{array}{l}\text { some: kidney, grl1, } \\
\text { intestind epithelium. } \\
\text { digestive tubules }\end{array}$} \\
\hline \multirow[t]{4}{*}{50 ppb } & $79-189$ & $5 d^{\prime}$ & M: kidney & $\begin{array}{l}\text { some; kidney, } \\
\text { gonad }\end{array}$ & & & \\
\hline & $79-190$ & PS $\sigma^{\prime \prime}$ & W: kindey & some; kidney & & & \\
\hline & $79-191$ & P5f & & & & & \\
\hline & $79-192$ & PS? & & & $\begin{array}{l}\text { some; digestive } \\
\text { tubules }\end{array}$ & & \\
\hline \multirow[t]{4}{*}{$100 \mathrm{ppb}$} & $79-213$ & P5d & & & $\begin{array}{l}\text { somp: digestuve } \\
\text { tubules }\end{array}$ & $\begin{array}{l}\text { some: digestive } \\
\text { tubules, scomach, } \\
\text { intestinal epr- } \\
\text { thel } 1 \text { i mom }\end{array}$ & $\begin{array}{l}\text { dutolys is of Leydig } \\
\text { cells }\end{array}$ \\
\hline & $79-214$ & LAC & & & & $\begin{array}{l}\text { some; digestive } \\
\text { tubules; intest inal } \\
\text { epithelium }\end{array}$ & $\begin{array}{l}\text { autolysis of Leydig } \\
\text { cells; vacuolization } \\
\text { of stomach epithelium }\end{array}$ \\
\hline & $79-215$ & 57 & & & $\begin{array}{l}\text { mall amount: } \\
\text { digestive tubules }\end{array}$ & $\begin{array}{l}\text { small amount; diges- } \\
\text { tive cubules }\end{array}$ & abscess on gill \\
\hline & $79-216$ & PS? & & & & $\begin{array}{l}\text { scrme; diges tive } \\
\text { qland, stomach }\end{array}$ & $\begin{array}{l}\text { vacualization of } \\
\text { stomach epithe lium }\end{array}$ \\
\hline
\end{tabular}




\section{Table 60. Summary of histological descriptions of the littleneck clam, Protothaca staminea, exposed to various amounts of chlorine for four months.}

$L A=$ late actlye gonad; $R=$ ripe gonad: $P S=$ partially spawned gonad: 5 = spent gonad: $M=$ metazoan parasite: G - gregarine-1ike parasite; $d^{\prime}=$ male: 8 : female

\begin{tabular}{|c|c|c|c|c|c|c|c|}
\hline $\begin{array}{l}\text { Chlorine } \\
\text { Concentration }\end{array}$ & $\begin{array}{l}\text { Specimen } \\
\text { Mnglier }\end{array}$ & $\begin{array}{l}\text { Gonad } \\
\text { Condition }\end{array}$ & Parasite & Leukøytos is & Metaplasia & Hecrosis & other \\
\hline \multirow[t]{4}{*}{ Control } & $79-97$ & Ró & & & & & \\
\hline & $79-99$ & P5? & & & $\begin{array}{l}\text { Some: digestive } \\
\text { tubuips }\end{array}$ & $\begin{array}{l}\text { some; kidney, } \\
\text { stamath }\end{array}$ & \\
\hline & $79-99$ & LA $\sigma^{\prime}$ & G: gill & some: gill & & & \\
\hline & $79-100$ & P5웅 & & & & $\begin{array}{l}\text { some; stomach } \\
\text { epi the } 1 \text { i um }\end{array}$ & abscess in kidney \\
\hline \multirow[t]{4}{*}{$6 \mathrm{ppb}$} & $79-121$ & $5 \%$ & $\begin{array}{l}\text { Unidentified in } \\
\text { gill }\end{array}$ & same; gill & & & \\
\hline & $79-122$ & $s^{f}$ & $\begin{array}{l}\text { Unidentified in } \\
\text { gill, kidney } \\
M_{;} \text {kidney }\end{array}$ & & & & \\
\hline & 79.123 & 57 & $\begin{array}{l}\text { M: kidney } \\
\text { Unidentified in } \\
\text { kidney }\end{array}$ & & $\begin{array}{l}\text { some; digestive } \\
\text { lubules }\end{array}$ & & $\begin{array}{l}\text { vacuolization of } \\
\text { stamach epithelfium }\end{array}$ \\
\hline & $79-124$ & $R d^{\prime}$ & $G ;$ foot & & $\begin{array}{l}\text { some: digestive } \\
\text { twhules }\end{array}$ & & $\begin{array}{l}\text { vacuolization of } \\
\text { stondch edithelium }\end{array}$ \\
\hline \multirow[t]{4}{*}{$12 \mathrm{gpb}$} & $79-145$ & $950^{\circ}$ & $\begin{array}{l}\text { G; pill } \\
\text { M; kidney }\end{array}$ & $\begin{array}{l}\text { in viscera around } \\
\text { cysts }\end{array}$ & & & \\
\hline & $79-146$ & P5ót & & & & & $\begin{array}{l}\text { bosophilic material } \\
\text { in gill }\end{array}$ \\
\hline & $79-147$ & PS\$? & $G ; g i l l$ & & $\begin{array}{l}\text { sane; digestive } \\
\text { tubules }\end{array}$ & $\begin{array}{l}\text { some: digestive } \\
\text { tubules }\end{array}$ & \\
\hline & $79-149$ & $R^{q}$ & M: kidney & & $\begin{array}{l}\text { some; digestive } \\
\text { tubules }\end{array}$ & $\begin{array}{l}\text { some: kidney, } \\
\text { glil, digestive } \\
\text { tutwies, stamact, } \\
\text { intest ine }\end{array}$ & \\
\hline \multirow[t]{4}{*}{$25 \mathrm{ppb}$} & $79-169$ & PSP & & & $\begin{array}{l}\text { same: digestive } \\
\text { tubules }\end{array}$ & $\begin{array}{l}\text { some; digestive } \\
\text { tubules }\end{array}$ & $\begin{array}{l}\text { vacuolization of } \\
\text { intestinal ept thelium }\end{array}$ \\
\hline & $79-170$ & Mo gonad & & general & $\begin{array}{l}\text { some; digestive } \\
\text { tubules }\end{array}$ & $\begin{array}{l}\text { some; digestive } \\
\text { tubules }\end{array}$ & \\
\hline & $79-171$ & sq & & general & $\begin{array}{l}\text { some; digestive } \\
\text { tubules }\end{array}$ & $\begin{array}{l}\text { some: digest tye } \\
\text { lubules }\end{array}$ & \\
\hline & $79-172$ & $P 5^{q}$ & & & & & \\
\hline \multirow[t]{4}{*}{$50 \mathrm{ppt}$} & $79-193$ & PS $\delta$ & N: kioney & $\begin{array}{l}\text { some; around } \\
\text { parasites }\end{array}$ & $\begin{array}{l}\text { some; digestive } \\
\text { tubules }\end{array}$ & 50 me; kidney & \\
\hline & $79-194$ & $5 ?$ & & general & $\begin{array}{l}\text { some; diggestive } \\
\text { tubules }\end{array}$ & kidney & $\begin{array}{l}\text { gonad follicles } \\
\text { present. but no } \\
\text { gametes }\end{array}$ \\
\hline & $79-195$ & p5? & & & $\begin{array}{l}\text { some; digestive } \\
\text { tubules }\end{array}$ & $\begin{array}{l}\text { some: stomach. } \\
\text { intest ines }\end{array}$ & \\
\hline & $79-196$ & PSF & Mi kidney & & $\begin{array}{l}\text { some; digestive } \\
\text { tubules }\end{array}$ & $\begin{array}{l}\text { some: digestive } \\
\text { tubules }\end{array}$ & $\begin{array}{l}\text { vacuolization of } \\
\text { standch eptthelium }\end{array}$ \\
\hline \multirow[t]{4}{*}{$100 \mathrm{ppb}$} & $79-217$ & Lat & M; kidney & $\begin{array}{l}\text { slight: in area } \\
\text { of porasite }\end{array}$ & & & \\
\hline & $79-218$ & LAd" & & & & & \\
\hline & $79-219$ & se & Mi kidney & & $\begin{array}{l}\text { some: digestive } \\
\text { tubules }\end{array}$ & $\begin{array}{l}\text { sone: digestive } \\
\text { tubules }\end{array}$ & \\
\hline & $29-220$ & PS? & & & & $\begin{array}{l}\text { some: stomach. } \\
\text { intesting }\end{array}$ & $\begin{array}{l}\text { vocuolization of } \\
\text { stomach and } \\
\text { intest ine }\end{array}$ \\
\hline
\end{tabular}


Table 6I. Summary of histological descriptions of the littleneck clam, Protothaca staminea, exposed to various amounts of chlorine for five months.

LA = iate active gonad; $R=$ ripe gonad: $P S=$ partially spawned gonad; $S$ = spent gonad; $M$ = metazoan parasite; G 2 gregarine-11ke parasite: $\gamma=$ male; $q=$ female

\begin{tabular}{|c|c|c|c|c|c|c|c|}
\hline $\begin{array}{l}\text { Chlorine } \\
\text { Concentration }\end{array}$ & $\begin{array}{l}\text { Specinen } \\
\text { Nhenber }\end{array}$ & $\begin{array}{l}\text { Gonad } \\
\text { condition }\end{array}$ & Parasite & Leukocytos is & Motaplasia & Mecrasis & Other \\
\hline \multirow[t]{3}{*}{ Control } & $79-701$ & PSd" & & & & $\begin{array}{l}\text { some; digestive } \\
\text { tubules }\end{array}$ & numerous abscesses \\
\hline & $79-102$ & PSd & & & $\begin{array}{l}\text { some; digestive } \\
\text { tutules }\end{array}$ & $\begin{array}{l}\text { some: digestive } \\
\text { tubules }\end{array}$ & \\
\hline & 79.103 & P5ó & & & $\begin{array}{l}\text { some: digestive } \\
\text { tubules }\end{array}$ & $\begin{array}{l}\text { sone; digestive } \\
\text { tubules }\end{array}$ & \\
\hline \multirow[t]{4}{*}{$6 \mathrm{ppb}$} & $79-125$ & So" & $\begin{array}{l}M_{\text {; k kidney (heavy }} \\
\text { infection) }\end{array}$ & general & $\begin{array}{l}\text { some: digestive } \\
\text { tubules }\end{array}$ & & $\begin{array}{l}\text { autolysis of Leydig } \\
\text { cells }\end{array}$ \\
\hline & $79-126$ & PSd & & & & $\begin{array}{l}\text { some; stomach, } \\
\text { kidney }\end{array}$ & $\begin{array}{l}\text { autolysis of Leydig } \\
\text { cells }\end{array}$ \\
\hline & $79-127$ & $s^{q}$ & H: kldney & kidney & & some; kidney & $\begin{array}{l}\text { vaculotzation of } \\
\text { stamach and intes- } \\
\text { tinal epithellum }\end{array}$ \\
\hline & $79-128$ & $56^{\circ}$ & M; kidney & $\begin{array}{l}\text { kidney; some } \\
\text { eosinophilic } \\
\text { leukocytes }\end{array}$ & & & \\
\hline \multirow[t]{4}{*}{$12 \mathrm{ppb}$} & $79-149$ & sot & M; kidney (heary) & vidney & & & \\
\hline & $79-150$ & pst? & $M_{;}$kidney & & $\begin{array}{l}\text { some; digestive } \\
\text { tubules }\end{array}$ & $\begin{array}{l}\text { some; digestive } \\
\text { tubules }\end{array}$ & \\
\hline & $79-151$ & $95^{\circ}$ & & general & & & \\
\hline & $79-752$ & sot & & & & $\begin{array}{l}\text { some; intestinal } \\
\text { ep ithel tum, stanach, } \\
\text { digest tre tubules }\end{array}$ & \\
\hline \multirow[t]{4}{*}{$25 \mathrm{ppb}$} & $79-173$ & $s+$ & $M ;$ Ktdney & general & & & \\
\hline & $79-174$ & PS? & G; foot & & & & \\
\hline & $79-175$ & $50 t$ & & & $\begin{array}{l}\text { some: digestive } \\
\text { tubuigs }\end{array}$ & & \\
\hline & $79-176$ & PS? & & general & & & \\
\hline \multirow[t]{4}{*}{$50 \mathrm{ppb}$} & $79-197$ & PSd & & & & & $\begin{array}{l}\text { autalys is of Leydig } \\
\text { cells; some vacuoliz- } \\
\text { dtion of intestinal } \\
\text { stanach epithelito }\end{array}$ \\
\hline & 19.198 & P5q & & & & $\begin{array}{l}\text { some; digestive } \\
\text { tubules }\end{array}$ & $\begin{array}{l}\text { yacuolization of } \\
\text { digestive tubules }\end{array}$ \\
\hline & $79-199$ & PSot & M: kidney & kidney & & $\begin{array}{l}\text { some; kidney, } \\
\text { extensive; digestive } \\
\text { tubules }\end{array}$ & \\
\hline & 79.200 & PSot & $M:$ kidney & $\begin{array}{l}\text { ridney (Extensive): } \\
\text { eosinophilic } \\
\text { leukocytes }\end{array}$ & & $\begin{array}{l}\text { some: digestive } \\
\text { tubules, portions } \\
\text { of intestine }\end{array}$ & $\begin{array}{l}\text { autolysis of Leydig } \\
\text { cells }\end{array}$ \\
\hline \multirow[t]{4}{*}{$100 \mathrm{ppb}$} & 79.221 & 59 & & & & & $\begin{array}{l}\text { autoiysis of Leydig } \\
\text { cells }\end{array}$ \\
\hline & $79-222$ & PSF & & & & & $\begin{array}{l}\text { yacuolization of } \\
\text { intestinal epithelium }\end{array}$ \\
\hline & $79-223$ & $s^{f}$ & & & & & \\
\hline & $79-224$ & s7 & & & & & $\begin{array}{l}\text { vacuolization of } \\
\text { stimach and } \\
\text { lntestinal opithelitum }\end{array}$ \\
\hline
\end{tabular}


Table 62. Summary of histological descriptions of the littleneck clam, Protothaca staminea, exposed to various amounts of chlorine for six months.

\begin{tabular}{|c|c|c|c|c|c|c|c|}
\hline $\begin{array}{l}\text { Dhorine } \\
\text { concentration }\end{array}$ & $\begin{array}{l}5 \text { specimen } \\
\text { Number }\end{array}$ & $\begin{array}{l}\text { Gonad } \\
\text { Condition }\end{array}$ & Parasite & Letuksoytosis & Metaplasia & Hecrosis & Other \\
\hline \multirow[t]{4}{*}{ control } & $79-105$ & $85^{\circ}$ & & & $\begin{array}{l}\text { occasional: } \\
\text { digestive tubules }\end{array}$ & & \\
\hline & 79.106 & $9 s^{8}$ & $\begin{array}{l}\text { G; mantle, gill } \\
\text { M: kidney }\end{array}$ & & & & \\
\hline & $79-107$ & $\mathrm{PS}^{8}$ & M; kiquey & & $\begin{array}{l}\text { some; digest ive } \\
\text { tubules }\end{array}$ & & \\
\hline & $79-108$ & Ró & G: gill & & & & \\
\hline \multirow[t]{4}{*}{$6 \mathrm{ppb}$} & $79-129$ & 59 & $M_{;}$kidney & kidney & & some; kidmey & \\
\hline & $79-130$ & $R^{q}$ & M: kidney & & & $\begin{array}{l}\text { consideratile; } \\
\text { kidney, digestive } \\
\text { tubules } \\
\text { some; stomach, } \\
\text { intest ind epi the lium }\end{array}$ & \\
\hline & $79-131$ & $\mathrm{Ps} \$$ & & & $\begin{array}{l}\text { some: diqestive } \\
\text { tubulps }\end{array}$ & $\begin{array}{l}\text { Some: kidney, } \\
\text { digestive tubulps }\end{array}$ & $\begin{array}{l}\text { sutolys is of Leysig } \\
\text { cellys }\end{array}$ \\
\hline & $79-132$ & $s^{2}$ & M: kidney & $\begin{array}{l}\text { kidney \{some } \\
\text { large eosinophilic } \\
\text { leukocytes\} }\end{array}$ & & some: stomach & \\
\hline \multirow[t]{4}{*}{$12 \mathrm{ppt}$} & $79-153$ & sq & & & $\begin{array}{l}\text { some: diqustive } \\
\text { tuoules }\end{array}$ & $\begin{array}{l}\text { some: digestive } \\
\text { tubules }\end{array}$ & $\begin{array}{l}\text { autolys is of Leydig } \\
\text { cells }\end{array}$ \\
\hline & 79.154 & $\mathrm{P5}^{7}$ & & & $\begin{array}{l}\text { some; digestive } \\
\text { tubules }\end{array}$ & $\begin{array}{l}\text { some, digestive } \\
\text { tubules }\end{array}$ & \\
\hline & $79-155$ & $s^{8}$ & $M_{i}$ kidney & $\begin{array}{l}\text { kidney, around } \\
\text { cysts }\end{array}$ & & & \\
\hline & $79-156$ & gonad & & general & & & $\begin{array}{l}\text { Gobrous deposits in } \\
\text { digestive gland }\end{array}$ \\
\hline \multirow[t]{4}{*}{$25 \mathrm{ppb}$} & $79-177$ & $5^{9}$ & $\mathrm{H} ; \mathrm{kidney}$ & & $\begin{array}{l}\text { some: orgestive } \\
\text { tubules } 5\end{array}$ & $\begin{array}{l}\text { Some: kidney. } \\
\text { digestive tubules }\end{array}$ & \\
\hline & $79-178$ & 57 & & & & & \\
\hline & $79-179$ & $15^{9}$ & $M_{;}$; kidney & & & & \\
\hline & $79-180$ & $50^{\circ}$ & & & $\begin{array}{l}\text { some: digest ive } \\
\text { tubules }\end{array}$ & $\begin{array}{l}\text { some: stomach } \\
\text { epithel i i }\end{array}$ & \\
\hline \multirow[t]{4}{*}{$50 \mathrm{pDt}$} & $79-201$ & PSo' & $\begin{array}{l}\text { Mi: kidney } \\
\text { L; mantle }\end{array}$ & $\begin{array}{l}\text { kidney; some large } \\
\text { eostnophitic cells }\end{array}$ & $\begin{array}{l}\text { some; digest the } \\
\text { tubuies }\end{array}$ & $\begin{array}{l}\text { some; kidmey. } \\
\text { digest ive tubules }\end{array}$ & $\begin{array}{l}\text { sutolys is of Leydig } \\
\text { cel } 115\end{array}$ \\
\hline & $79-202$ & 58 & & & $\begin{array}{l}\text { Some; digestive } \\
\text { tubules }\end{array}$ & $\begin{array}{l}\text { extensive: diges- } \\
\text { ive tubuless: } \\
\text { some. intestine. } \\
\text { stomith }\end{array}$ & $\begin{array}{l}\text { Somme yacuolization of } \\
\text { stamach epithelinum: } \\
\text { sutolysis of Leydig } \\
\text { cells }\end{array}$ \\
\hline & $79-203$ & $50^{\circ}$ & $M_{;}$kidney & viscera & $\begin{array}{l}\text { some; digestive } \\
\text { tubules }\end{array}$ & $\begin{array}{l}\text { kidney; some } \\
\text { digest ive tubules }\end{array}$ & $\begin{array}{l}\text { autolysis of Leydig } \\
\text { cells }\end{array}$ \\
\hline & $79-204$ & ps\$ & & mild: gills & $\begin{array}{l}\text { some: digestive } \\
\text { tubules }\end{array}$ & $\begin{array}{l}\text { some; stomath, } \\
\text { intestine }\end{array}$ & $\begin{array}{l}\text { sutglys is of Leysig } \\
\text { cells: abscesses in } \\
\text { foot; vacuolization } \\
\text { of stomach, in test inal } \\
\text { epitheli ium }\end{array}$ \\
\hline \multirow[t]{4}{*}{$100 \mathrm{pPb}$} & 79-225 & $5^{7}$ & & general & $\begin{array}{l}\text { some; diquestive' } \\
\text { cells }\end{array}$ & $\begin{array}{l}\text { some: stomach, } \\
\text { in tes tine, } k \text { toney }\end{array}$ & $\begin{array}{l}\text { autolysis of Leydig } \\
\text { cells }\end{array}$ \\
\hline & $79-226$ & $\mathrm{P5}^{9}$ & & & & $\begin{array}{l}\text { gil15; some } \\
\text { digastive tubules }\end{array}$ & $\begin{array}{l}\text { yacuoliation of stamach, } \\
\text { intestinal enpithelium. } \\
\text { digestive tubules }\end{array}$ \\
\hline & $79-227$ & Rd & & & & 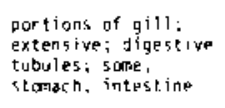 & \\
\hline & $79-228$ & $\mathrm{LAO}^{\circ}$ & & generd 1 & & $\begin{array}{l}\text { sone; intestine, } \\
\text { gill }\end{array}$ & $\begin{array}{l}\text { vacuolifation of } \\
\text { intestinal epithelium }\end{array}$ \\
\hline
\end{tabular}


Table 63. Summary of histopathological observations of 1 ittleneck clams, Protothaca staminea exposed to various CPO concentrations for up to six months.

\begin{tabular}{|c|c|c|c|c|c|c|c|c|}
\hline $\begin{array}{l}\text { Number : } \\
\text { Wonths }\end{array}$ & Exposed & $\begin{array}{c}\text { CPO } \\
\text { Concentrations }(\mu g / 1)\end{array}$ & $\begin{array}{l}\text { No. of Clams } \\
\text { in-Sanple }\end{array}$ & Leukocytosis & $\begin{array}{c}\text { Number of clars } \\
\text { Metaplasia }\end{array}$ & $\begin{array}{l}\text { 1th histop: } \\
\text { Mecrosis }\end{array}$ & $\begin{array}{l}\text { thological conditfons } \\
\text { Yacuolization of } \\
\text { Digestive Tissues }\end{array}$ & $\begin{array}{c}\text { Abscesses } \\
\text { Auttolysis, etc. }\end{array}$ \\
\hline Initial & Sample & 0 & 10 & 0 & 2 & 5 & 0 & 0 \\
\hline & 1 & $\begin{array}{r}0 \\
6 \\
12 \\
25 \\
50 \\
100\end{array}$ & $\begin{array}{l}4 \\
4 \\
4 \\
4 \\
4 \\
4\end{array}$ & $\begin{array}{l}0 \\
1 \\
0 \\
1 \\
1 \\
1\end{array}$ & $\begin{array}{l}0 \\
2 \\
0 \\
0 \\
0 \\
0\end{array}$ & $\begin{array}{l}3 \\
3 \\
4 \\
4 \\
4 \\
4\end{array}$ & $\begin{array}{l}0 \\
0 \\
0 \\
0 \\
0 \\
0\end{array}$ & $\begin{array}{l}0 \\
4 \\
0 \\
1 \\
0 \\
1\end{array}$ \\
\hline & 2 & $\begin{array}{r}0 \\
6 \\
12 \\
25 \\
50 \\
100\end{array}$ & $\begin{array}{l}4 \\
4 \\
4 \\
4\end{array}$ & $\begin{array}{l}1 \\
1 \\
0 \\
2 \\
2 \\
3\end{array}$ & $\begin{array}{l}0 \\
0 \\
1 \\
0 \\
0 \\
0\end{array}$ & $\begin{array}{l}1 \\
0 \\
2 \\
3 \\
3 \\
3\end{array}$ & $\begin{array}{l}0 \\
0 \\
0 \\
0 \\
0 \\
3\end{array}$ & $\begin{array}{l}0 \\
0 \\
1 \\
2 \\
1 \\
3\end{array}$ \\
\hline & 3 & $\begin{array}{r}0 \\
5 \\
12 \\
25 \\
50 \\
100\end{array}$ & $\begin{array}{l}4 \\
4 \\
4 \\
4 \\
4 \\
4\end{array}$ & $\begin{array}{l}7 \\
\vdots \\
0 \\
2 \\
0\end{array}$ & $\begin{array}{l}0 \\
0 \\
0 \\
1 \\
1 \\
2\end{array}$ & $\begin{array}{l}0 \\
0 \\
0 \\
2 \\
0 \\
4\end{array}$ & $\begin{array}{l}0 \\
0 \\
0 \\
0 \\
0 \\
2\end{array}$ & $\begin{array}{l}0 \\
1 \\
1 \\
0 \\
0 \\
3\end{array}$ \\
\hline & 4 & $\begin{array}{r}0 \\
5 \\
12 \\
25 \\
50 \\
100\end{array}$ & $\begin{array}{l}4 \\
4 \\
4 \\
4 \\
4\end{array}$ & $\begin{array}{l}1 \\
1 \\
1 \\
2 \\
2 \\
1\end{array}$ & $\begin{array}{l}1 \\
2 \\
2 \\
3 \\
4 \\
1\end{array}$ & $\begin{array}{l}2 \\
0 \\
3 \\
3 \\
3 \\
2\end{array}$ & $\begin{array}{l}0 \\
2 \\
0 \\
1 \\
1 \\
1\end{array}$ & $\begin{array}{l}3 \\
1 \\
1 \\
0 \\
1 \\
0\end{array}$ \\
\hline & 5 & $\begin{array}{r}0 \\
6 \\
12 \\
25 \\
50 \\
100\end{array}$ & $\begin{array}{l}3 \\
4 \\
4 \\
4 \\
4 \\
4\end{array}$ & $\begin{array}{l}0 \\
3 \\
2 \\
2 \\
1 \\
0\end{array}$ & $\begin{array}{l}2 \\
1 \\
1 \\
1 \\
0 \\
0\end{array}$ & $\begin{array}{l}3 \\
2 \\
2 \\
0 \\
3 \\
0\end{array}$ & $\begin{array}{l}0 \\
1 \\
0 \\
0 \\
2 \\
2\end{array}$ & $\begin{array}{l}1 \\
2 \\
0 \\
0 \\
2 \\
1\end{array}$ \\
\hline & 6 & $\begin{array}{r}0 \\
6 \\
12 \\
25 \\
50 \\
100\end{array}$ & $\begin{array}{l}4 \\
4 \\
4 \\
4 \\
4\end{array}$ & $\begin{array}{l}0 \\
2 \\
1 \\
0 \\
3 \\
2\end{array}$ & $\begin{array}{l}2 \\
1 \\
2 \\
2 \\
4 \\
1\end{array}$ & $\begin{array}{l}0 \\
4 \\
2 \\
2 \\
4 \\
4\end{array}$ & $\begin{array}{l}0 \\
0 \\
0 \\
0 \\
2 \\
2\end{array}$ & $\begin{array}{l}0 \\
1 \\
2 \\
0 \\
1 \\
1\end{array}$ \\
\hline
\end{tabular}


Table 64. Bromoform concentrations in clams exposed to chlorinated sea water containing sublethal concentrations of chlorine produced oxidant (CPO).

\begin{tabular}{|c|c|c|c|c|c|c|c|c|}
\hline $\mathrm{CPO}$ & -2 & & $--n$ & $\mathrm{OF} H$ & RVEST & & & \\
\hline$\mu \mathrm{g} / \ell$ & $3 / 1$ & $4 / 3$ & $\begin{array}{c}5 / 2 \\
n g ~ \\
\text { Bromo }\end{array}$ & $\begin{array}{l}5 / 30 \\
\text { orm/gram }\end{array}$ & $\begin{array}{l}6 / 29 \\
\text { tissue }\end{array}$ & $\begin{array}{l}8 / 1 \\
\text { wet wt. }\end{array}$ & $9 / 5$ & $11 / 8$ \\
\hline Control & $0^{\star}$ & $\begin{array}{r}0 \\
226 \\
107 \\
55\end{array}$ & $\begin{array}{l}0 \\
6 \\
0 \\
0\end{array}$ & $\begin{array}{r}12 \\
5 \\
0\end{array}$ & $g$ & $g$ & $\begin{array}{r}0 \\
10 \\
0\end{array}$ & $\begin{array}{l}1 \\
9 \\
0^{\mathrm{a}}\end{array}$ \\
\hline 6 & & $\begin{array}{r}97 \\
166 \\
0 \\
140\end{array}$ & $\begin{array}{r}20 \\
15 \\
0 \\
0\end{array}$ & $\begin{array}{r}0 \\
0 \\
0 \\
11\end{array}$ & $\begin{array}{l}0 \\
0 \\
0 \\
0\end{array}$ & $\begin{array}{l}2 \\
0 \\
0 \\
0\end{array}$ & $\begin{array}{l}0 \\
0 \\
0 \\
0\end{array}$ & $0^{b}$ \\
\hline 12 & & $\begin{array}{r}33 \\
183 \\
238 \\
296\end{array}$ & $\begin{array}{r}56 \\
9 \\
0\end{array}$ & $\begin{array}{r}2 \\
169 \\
0 \\
9\end{array}$ & $\begin{array}{l}0 \\
0 \\
0 \\
0\end{array}$ & $\begin{array}{r}0 \\
10 \\
14 \\
18\end{array}$ & $\begin{array}{r}1 \\
9 \\
3 \\
20 \\
0\end{array}$ & $\begin{array}{r}2 \\
{ }^{40} \\
{ }_{0} \mathrm{C}\end{array}$ \\
\hline 25 & & $\begin{array}{r}24 \\
123 \\
74 \\
42\end{array}$ & $\begin{array}{l}72 \\
13 \\
80 \\
39\end{array}$ & $\begin{array}{r}348 \\
26 \\
20 \\
9\end{array}$ & $\begin{array}{r}17 \\
0 \\
0 \\
35\end{array}$ & $\begin{array}{l}18 \\
14 \\
14 \\
17\end{array}$ & $\begin{array}{r}0 \\
0 \\
208\end{array}$ & $\begin{array}{l}3 \\
2 \\
2 \\
0\end{array}$ \\
\hline 50 & & $\begin{array}{r}107 \\
34 \\
97 \\
352\end{array}$ & $\begin{array}{r}21 \\
16 \\
6 \\
82\end{array}$ & $\begin{array}{r}7 \\
11 \\
1 \\
8\end{array}$ & $\begin{array}{l}13 \\
44 \\
25 \\
41\end{array}$ & $\begin{array}{r}4 \\
0 \\
0 \\
14\end{array}$ & $\begin{array}{l}0 \\
0 \\
0 \\
0\end{array}$ & ${ }_{0}^{2} \mathrm{e}$ \\
\hline 100 & & $\begin{array}{r}72 \\
95 \\
103 \\
89\end{array}$ & $\begin{array}{r}150 \\
153 \\
64 \\
60\end{array}$ & $\begin{array}{l}14 \\
43 \\
46 \\
32\end{array}$ & $g$ & $\begin{array}{r}0 \\
22 \\
26 \\
8\end{array}$ & $\begin{array}{l}6 \\
2 \\
0 \\
2\end{array}$ & $\begin{array}{c}18 \\
18 \\
22 \\
33 \\
0 \\
0\end{array}$ \\
\hline
\end{tabular}

* Represents 13 individuals

a Represents 9 individuals

b Represents 11 individuals

C Represents 4 individuals

depresents 7 individuals

e Represents 6 individuals

f Represents 4 individuals

go sample 


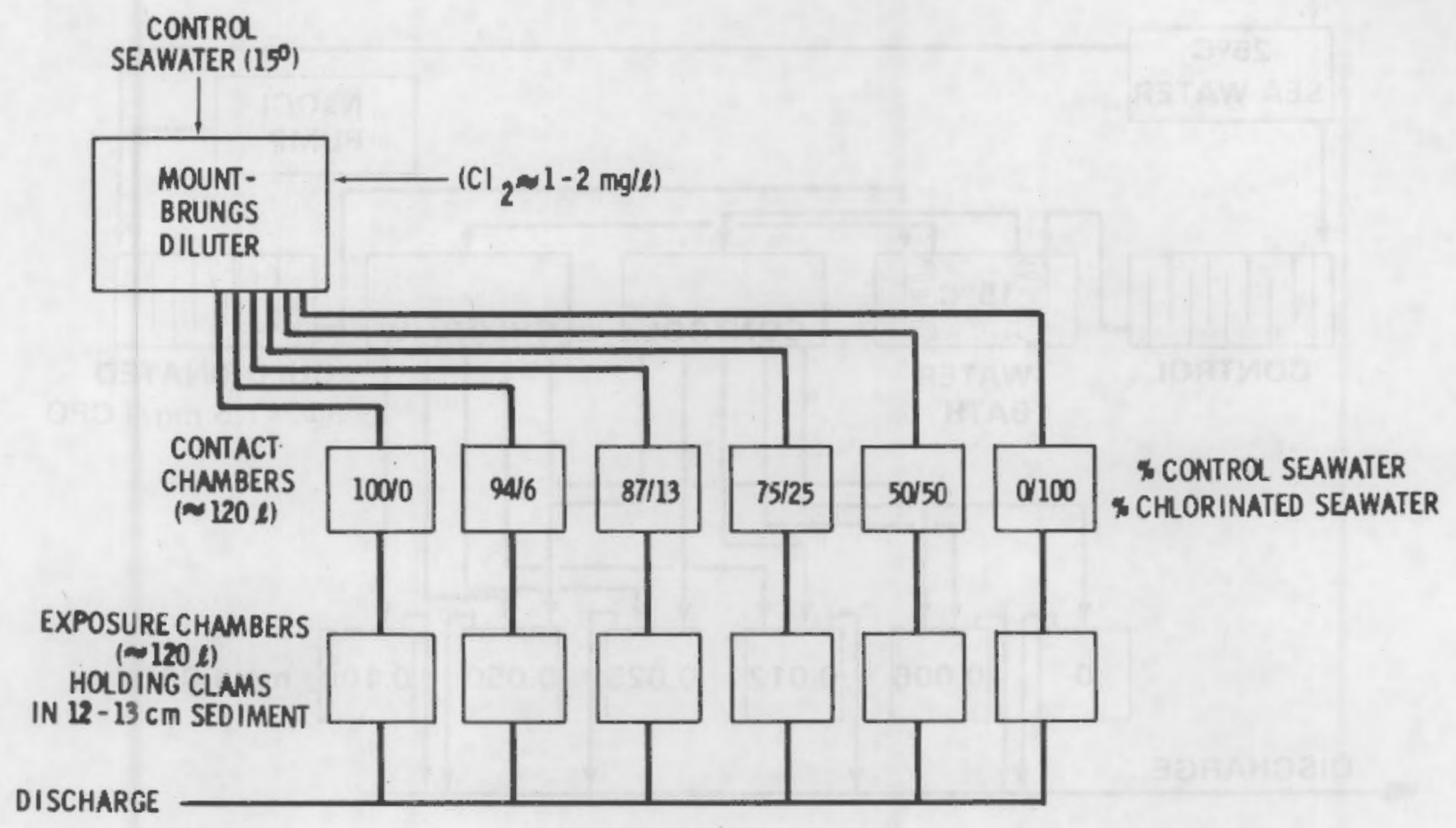

Figure 1. Exposure system used for Protothaca staminea in the first growth experiments. 


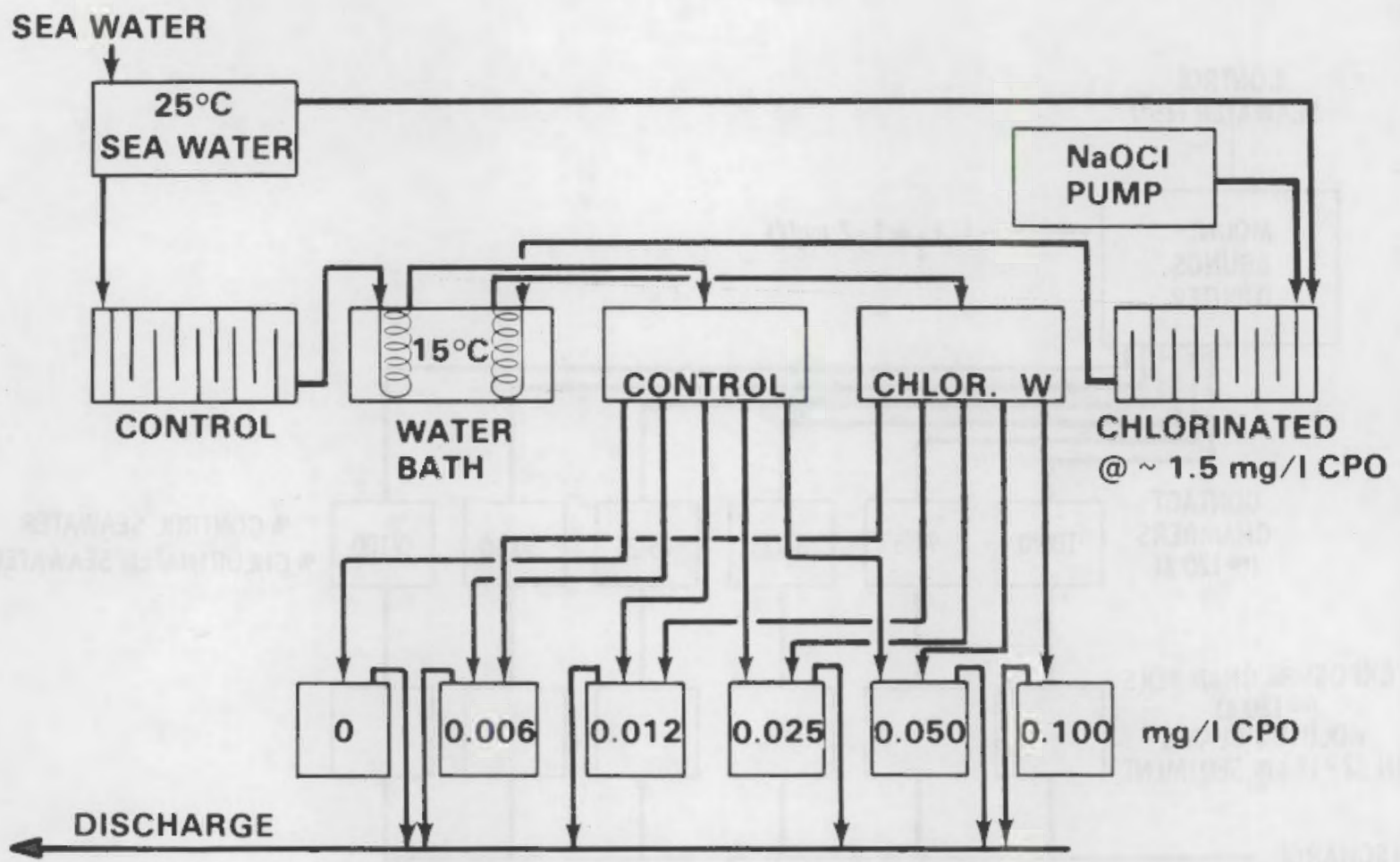

Figure 2. Exposure system used for Protothaca staminea in the second growth experiments. 


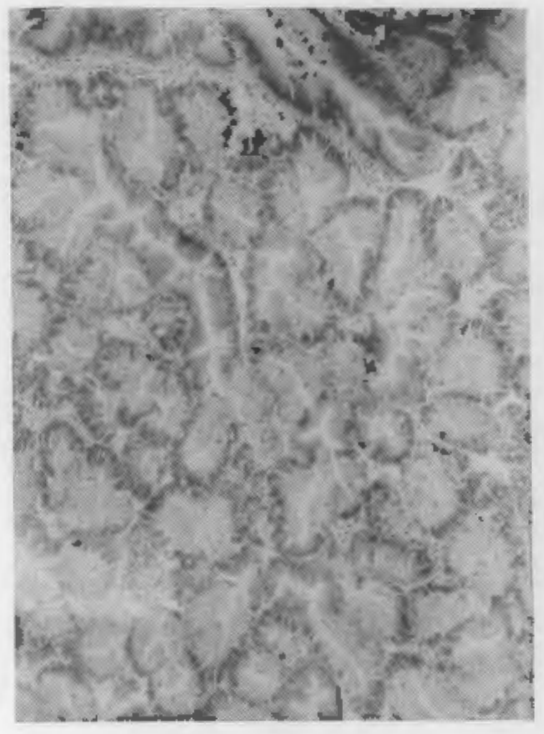

A. NORMAL DIGESTIVE TUBULE EPITHELIUM



C. NECROTIC STOMACH EPITHELIUM (LEFT) AND METAPLASTIC DIGESTIVE TUBULE EPITHELIUM (RIGHT) OF CLAM EXPOSED FOR 6 MONTHS AT 50 pDb CPO

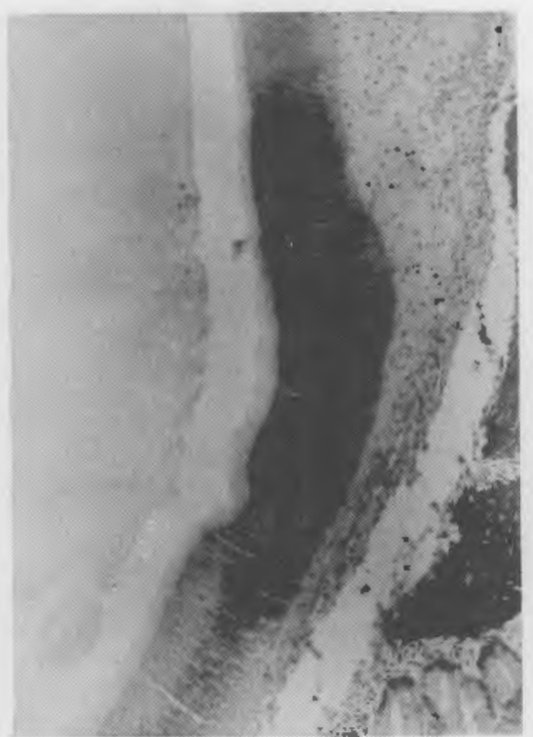

B. NORMAL STOMACH EPITHELIUM

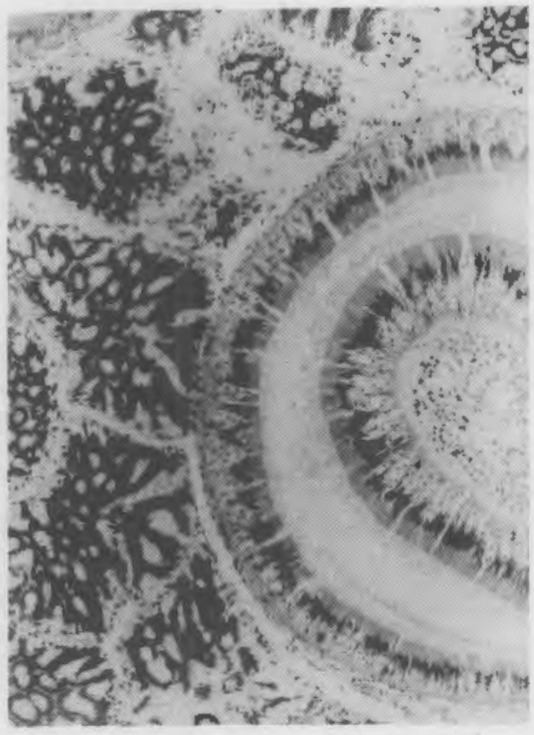

D. VACUOLIZATION OF INTESTINAL EPITHELIUM OF CLAM EXPOSED FOR 6 MONTHS AT $100 \mathrm{pPD}$ CPO. NOTE LEUKOCYTIC INFILTRATION INTO EPITHELIUM.

Figure 3. Photomicrographs of sections through the littleneck clam, Protothaca staminea. 



\section{DISTRIBUTION}

No, of

Copies

OFFSITE

A. A. Churm

DOE Patent Division

$9800 \mathrm{~S}$. Cass Avenue

Argonne, IL 60439

10 Dr. Phillip R. Reed

Bob Samworth

John C. Lehr

U.S. Nuclear Regulatory Commission

7915 Eastern Avenue

Silver Springs, MD 20901

265 NRC Division of Technical Information and Document Control

Washington, DC 20555

2 DOE Technical Information Center

D. R. Anderson

2521 N. 40th, No. 1

Seattle, WA 98103

Dr. Robert Broxton

Electric Power Research Institute

PO Box 10412

Palo Alto, CA 94303

Dr. Dennis T. Burton

Academy of Natural Sciences of Philadelphia

Benedict Estuarine Research Laboratory

Benedict, MD 20612
No. of

Copies

Dr. Will Davis

U.S.E.P.A.

Gulf Breeze Environmental

Research Laboratory

Wadmalaw Island, SC 19487

Dr. R. E. Hillman

W. F. Clapp Laboratories

Washington Street, P.0. Box AH

Duxbury, MA 02332

Or. Donald Johnson

Dept. Environmental Sciences and Engineering School of Public Health

University of North Carolina

ChapeT Hill, NC 27514

Dr. George R. Helz

Chemistry Dept.

University of Maryland

College Park, MD 20742

Dr. Milton H. Lietzke

PO Box $X$

Oak Ridge National Laboratory

oak Ridge, TN 37830

Dr. J. S. Mattice

PO Box $X$

Oak Ridge National Laboratory

Oak Ridge, TN 37830

Dr. John Lum

U.S.E.P.A.

401 M Street S.W.

WH552

Washington, DC 20460 
No. of

Copies

Or. Roy Nakatani

Or. Quentin J. Stober

FRI $\mathrm{WH}-10$

University of Washington

SeattTe, WA 98195

Dr. Thomas 0. Thatcher

Rt 2 Box 113

Sequim, WA 98382

F. C. Tone

Florida Marine Research Facility

Sailfish Drive

Ponce Inlet

Daytona Beach, FL 32019

Ronald L. Raschke

U.S. EPA

S.E. Water Laboratory

Bailey Road

Athens, GA 30601
No. of

Copies

ONSITE

50 Pacific Northwest Laboratory

RM Bean (32)

CI Gibson

KE Harding (4)

DC Mann

TL Page

RG Riley

RE Schirmer

NM Sherer

RE Wiidung

Publishing Coordination (2)

Technical Information (5) 



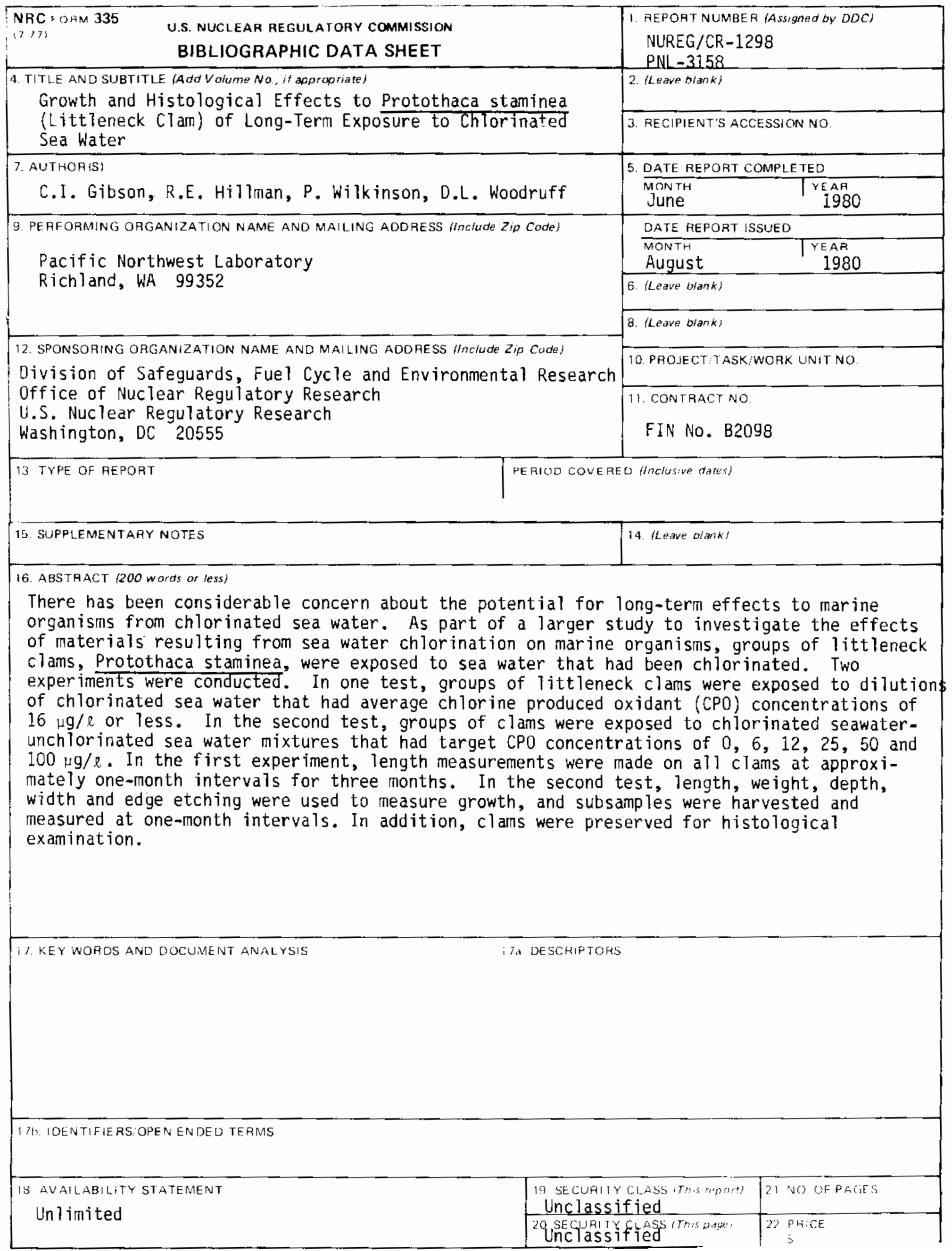


